\title{
Optimization of an Optical Thermal Absorber Plasmonic Metamaterial: Understanding the Two Particle Interactions
}

Anitesh Anand Lal

Follow this and additional works at: https://researchrepository.wvu.edu/etd

\section{Recommended Citation}

Lal, Anitesh Anand, "Optimization of an Optical Thermal Absorber Plasmonic Metamaterial: Understanding the Two Particle Interactions" (2014). Graduate Theses, Dissertations, and Problem Reports. 7325.

https://researchrepository.wvu.edu/etd/7325

This Thesis is protected by copyright and/or related rights. It has been brought to you by the The Research Repository @ WVU with permission from the rights-holder(s). You are free to use this Thesis in any way that is permitted by the copyright and related rights legislation that applies to your use. For other uses you must obtain permission from the rights-holder(s) directly, unless additional rights are indicated by a Creative Commons license in the record and/ or on the work itself. This Thesis has been accepted for inclusion in WVU Graduate Theses, Dissertations, and Problem Reports collection by an authorized administrator of The Research Repository @ WVU. For more information, please contact researchrepository@mail.wvu.edu. 


\title{
Optimization of an Optical Thermal Absorber Plasmonic Metamaterial: Understanding the Two Particle Interactions
}

\author{
Anitesh Anand Lal \\ Thesis submitted \\ to the Benjamin M. Statler College of \\ Engineering and Mineral Resources \\ at West Virginia University \\ in partial fulfillment of the requirements for the degree of \\ Master of Science \\ in \\ Mechanical Engineering
}

Terence Musho, Ph.D., Chair

Osama Mukdadi, Ph.D.

Nick Wu, Ph.D.

Department of Mechanical and Aerospace Engineering

Morgantown, West Virginia

May, 2014

Keywords: plasmonics, fractals, metamaterials, sub-wavelengths, resonance Copyright 2014 Anitesh Anand Lal 
All rights reserved

INFORMATION TO ALL USERS

The quality of this reproduction is dependent upon the quality of the copy submitted.

In the unlikely event that the author did not send a complete manuscript and there are missing pages, these will be noted. Also, if material had to be removed, a note will indicate the deletion.

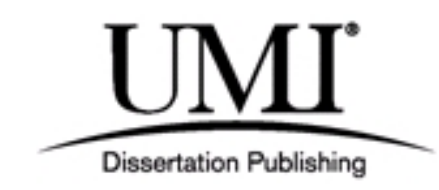

\section{UMI 1554841}

Published by ProQuest LLC (2014). Copyright in the Dissertation held by the Author.

Microform Edition () ProQuest LLC.

All rights reserved. This work is protected against unauthorized copying under Title 17, United States Code

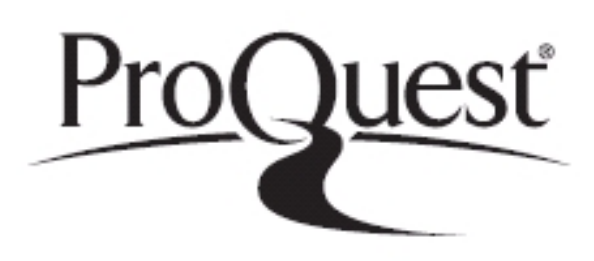

ProQuest LLC.

789 East Eisenhower Parkway

P.O. Box 1346

Ann Arbor, MI 48106 - 1346 


\title{
ABSTRACT \\ Optimization of a Nearly Total Optical Absorption Plasmonic Metamaterial: Understanding the Interaction Effects
}

\author{
Anitesh Anand Lal
}

In the recent years, the emergence of electromagnetic metamaterials (MMs) have ushered in an exciting new field with many promising applications in direct energy conversion. One of the more promising applications of MMs is the ability to fully harness the solar energy through the design of a perfect electromagnetic absorber. Electromagnetic MMs posses the ability to manipulate a material's response resulting in augmented properties such as negative index of refraction, artificial permittivity, and permeability. One of the proven methods for the construction of electromagnetic metamaterials is to use a metal-dielectric composite whereby the electromagnetic response is governed by oscillating surface plasmons and the geometry of the dielectric environment. However, the majority of these materials exhibit a peak absorptivity at a single frequency, therefore, a deep understanding of their response becomes necessary to target approaches to broadening the absorptivity.

In this research the reflectance, transmittance, absorbance, and heat generation in a square nano-antenna composed of a metal-dielectric-metal construction was investigated. The wavelength of interest was in the visible regime from 390-700nm (430-790 THz). The dual focus of this research was 1 ) the development of metamateial with high absorbance characteristics and 2) the development of a broadband response. The focus was on tailoring the particle geometry and dielectric environment. The nano-antenna was constructed from a silver nano-particle situated on top of a controlled thickness $\mathrm{Al}_{2} \mathrm{O}_{3}$ dielectric material followed by a thick silver ground plane. For this study a transverse electric (TE) plane wave was propagated in the negative $\mathrm{z}$ direction and the resulting scattered parameters were monitored. In order to evaluate the electromagnetic response (absorbance) at the sub-wavelength scales, a finite-difference time-domain (FDTD) method was implemented. In addition, the interaction from the neighboring particles en route to a perfect broadband absorber was studied. Our simulation results indicate a complex response between neighboring particles that could be categorized into four independent effective interactions. Furthermore, by controlling the distance between particles, the absorptivity achieved an increase by seven fold. 
To my mother and grandfather,

\section{Anita Devi and Karan Prasad}

$$
\text { and }
$$

my high school principal, Mr. Ben Salacakau 


\section{ACKNOWLEDGMENTS}

During the course of my graduate curriculum, I had the pleasure of interacting with many highly intelligent and talented people. Without these people, this achievement would not have been accomplished. I would like to extend my sincere gratitude to each and all of them.

Foremost, I am deeply grateful and indebted to Doctor Terence D. Musho for his unrelenting support. He has provided me guidance, advice and encouragement in each facet of my master's program. In addition to his expertise and suggestions that were crucial to my research work, his enthusiasm as well as ambition has motivated me to learn new ideas.

Also I would like to thank all my committee members Doctor Nick Wu and Doctor Osama Mukdadi for providing me with insightful comments. They have contributed a lot with their time in reviewing this thesis and their helpful advice for improvement.

Finally, my sincere gratitude also goes for my undergraduate advisor, Doctor Dan Mazilu. He always motivated me to pursue higher education for betterment and success in life. He has always been there for me as an advisor, a friend and a mentor and assisted me in anyway possible. 


\section{TABLE OF CONTENTS}

Page

DEDICATION .................. . iii

ACKNOWLEDGMENTS. . . . . . . . . . . . . iv

LIST OF FIGURES . . . . . . . . . . . . . . . . . xiv

LIST OF TABLES . . . . . . . . . . . . . . . . $\mathrm{xv}$

LIST OF SYMBOLS . . . . . . . . . . . . . . . . xvi

\section{Chapter}

1. INTRODUCTION . . . . . . . . . . . . . . . . 1

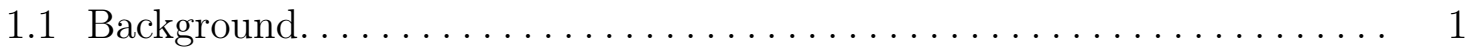

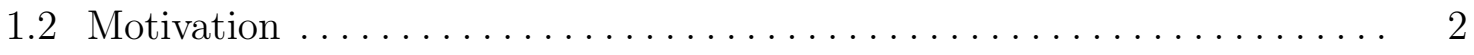

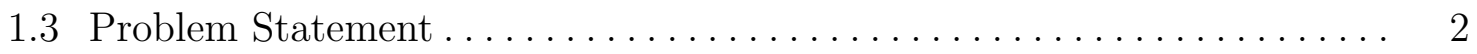

1.4 Research Objective ......................... 4

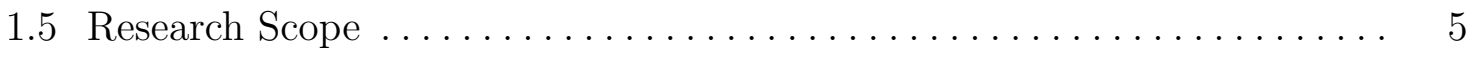

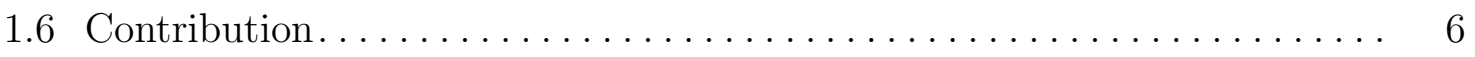

2. LITERATURE REVIEW . . . . . . . . . . . . . . . 7

2.1 Maximum amount of energy absorbed from the sun $\ldots \ldots \ldots \ldots \ldots \ldots$

2.1 .1 Energy of photon $\ldots \ldots \ldots \ldots \ldots \ldots \ldots \ldots \ldots \ldots \ldots \ldots \ldots \ldots \ldots \ldots \ldots$

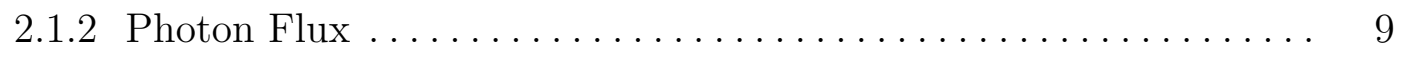

2.1 .3 Power Density ........................... 9

2.1 .4 Spectral Irradiance . . . . . . . . . . . . . . . . . . . 9

2.1 .5 Radiant Power Density . . . . . . . . . . . . . . . . . . 10

2.1.6 Sun's Spectral Irradiance modeled as a Blackbody Radiator . . . . . 10

2.1.7 Total Solar Radiant Power Density . . . . . . . . . . . . . . . . . . . 13

2.1 .8 Solar Radiation in Space. . . . . . . . . . . . . . . . . . . 13 


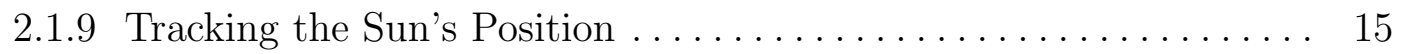

2.1.9.1 Declination Angle ............................. 15

2.1.9.2 Elevation Angle ........................... 15

2.1.9.3 Azimuth Angle............................ 16

2.2 Optical Properties of Metal Nanostructures . . . . . . . . . . . . . . . 17

2.3 Emergence of Metamaterials $(\mathrm{MMs}) \ldots \ldots \ldots \ldots \ldots \ldots \ldots \ldots \ldots \ldots \ldots$

2.4 Applications of Metamaterials......................... 21

2.4 .1 Superlens ................................ 21

2.4.2 Biosensor and Cellulor Detector $\ldots \ldots \ldots \ldots \ldots \ldots \ldots \ldots \ldots . \ldots \ldots$

2.4 .3 Invisible Cloaks................................ 24

2.5 Construction of Electromagnetic Metamaterials ............... 25

2.5.1 Electromagnetic Wave Absorption Theory................... 25

2.5.2 Surface Plasmons . . . . . . . . . . . . . . . . . . . . . . 25

2.5.2.1 Propagating Surface Plasmons ................. 26

2.5.2.2 Localized Surface Plasmons.................... 27

2.5 .3 Noble Metals .................................. 28

2.5.3.1 Drude Model ............................... 29

2.5.4 Effective Medium Theory $\ldots \ldots \ldots \ldots \ldots \ldots \ldots \ldots \ldots \ldots \ldots \ldots$

2.5.4.1 Alternatives to Noble Metals...................... . 31

2.5.5 Influence of dielectric environment $\ldots \ldots \ldots \ldots \ldots \ldots \ldots \ldots \ldots \ldots \ldots$

2.6 Absorptive and dispersive Materials...................... 33

2.6 .1 Pyonting Theorem .............................. 35

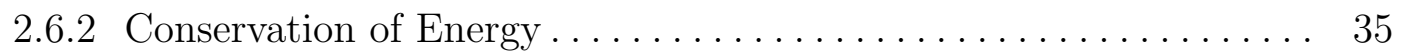

2.7 Narrow-Band Perfect Metamaterial Absorber (PAs)................. 36

2.7.1 Perfect Impedance Matching . . . . . . . . . . . . . . . . . . . 37

2.7.2 Resonant Absorbers ............................ 38

2.7.3 Previous Modeling Designs ....................... 38

2.7.3.1 Split Ring Resonators.......................... 38

2.7.3.2 Parallel Cut Wires............................. 39

2.7.3.3 Even Leaf Shapes in Nature ...................... 40

2.8 Broadband Metamaterial Absorber ........................ 40

2.8.1 Multiple sub-cells within the unit cell................... 40

2.8.2 Fractal plasmonic metamaterial broadband aborber .......... 41 
3. FINITE-DIFFERENCE TIME-DOMAIN (FDTD) SIMULATION. . 43

3.1 Maxwell's Equations................................ 43

3.1.1 Maxwell's Equations in Noble Metals...................... 44

3.1.2 Maxwell's Equations in Dielectrics ..................... 45

3.2 Numerical Solutions for Maxwell's equations .................. 46

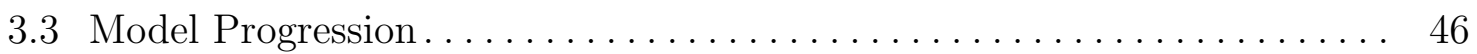

3.3.1 Constructing the CAD model. ...................... 46

3.3.2 Determine the Material Properties .................... 47

3.3.3 Periodic Boundary Conditions ....................... 48

3.3.4 Plane Wave Excitation and Wave Ports................... 49

3.3 .5 FDTD simulation ............................. 49

3.3.5.1 Time Domain Solver ......................... 49

3.3.5.2 Frequency Domain Solver..................... 50

3.3 .6 Field Monitors. . . . . . . . . . . . . . . . . . . . . . . . . . .... 51

3.3.7 Scattering Parameters or S-parameters . .............. 51

3.3 .8 Absorption Calculation ............................ 53

3.3.9 Model Modification ................................ 54

4. COMPUTATIONAL RESULTS. . . . . . . . . . . . . 55

4.1 Design Rationale ..................................... 55

4.1.1 Perfect Peak Absorbers ................................. 55

4.1.2 Perfect absorber and its progress to broadband absorber ........ 57

4.1.3 Current Fractal Design ............................ 59

4.2 Preliminary Unit Cell Plasmonic Meta-Material Device Design ....... . . 60

4.2 .1 First Order Absorber.............................. 60

4.2.2 Resonance information for the first order absorber ........... 62

4.2 .3 Adaptive Mesh Refinement............................ 65

4.2.4 Polarization Independence of the First Order Absorber ........ 66

4.2.5 Comparison of different unit-celled nano-antennas . . . . . . . . . 67

4.3 Influence of nano-particle width and dielectric thickness . . . . . . . . . . 69

4.4 Interaction effects due to neighboring particles ............... 74

4.5 Effects of Magnetic and Electric Resonances ................... 84

4.6 Resonance information for the second order absorber............. 92

4.7 Resonance information for the third order absorber .............. 95 
5. CONCLUSION. . . . . . . . . . . . . . 97

5.1 Summary of Research ............................... 97

5.2 Future Directions.................................. 99

REFERENCES. . . . . . . . . . . . . . . . . 101 


\section{LIST OF FIGURES}

Figure 2.1 A visualization of the visible spectrum as a subset of the electromagnetic radiation $[1]$.

Figure 2.2 The $\bar{E}$ and $\bar{H}$ vectors for a plane electromagnetic wave propagating in the positive direction along the $\mathrm{x}$-axis. The direction of propagation is a result of the cross between the $\bar{E} \times \bar{H}$ vectors. The two vectors are in phase, but perpendicular to each other $[2,1] \ldots \ldots \ldots \ldots \ldots \ldots$

Figure 2.3 Illustration of solar spectral irradiance as a function of wavelength regenerated from data obtained from National Institute of Standards

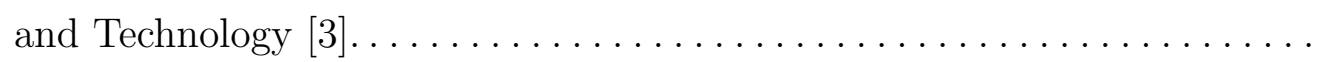

Figure 2.4 Relative position of the earth from the sun and their specific

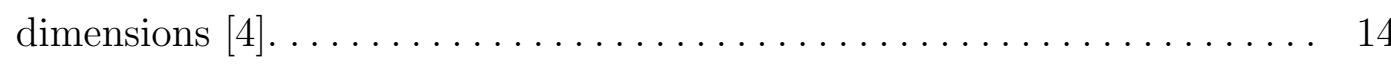

Figure 2.5 Illustration of light and nano-metallic structures interaction taken at the train station mall in Saint Loius Missouri, 2011. . . . . . . . . . . . . . 17

Figure 2.6 Historical illustrations of light-metal interactions: Lycurgus cup shown as green while reflecting light and green while transmitting light and the church window glasses in Mainz $[5] \ldots \ldots \ldots \ldots \ldots \ldots \ldots \ldots$

Figure 2.7 $\mathrm{A}$ ) Depicts a periodic arrangement of a metamaterial composite in comparison to Figure $2.7 \mathrm{~B}$ ) which depicts a non-periodic arrangement metamaterial composite [6].

Figure 2.8 A comparison of typical materials found in nature in relation to Left-handed materials [6].

Figure 2.9 A cuboidal shaped super high resolution superlens operating using wireless power transfer $[7]$ and hence, detect cancerous cells. . . . . . . . .

Figure 2.10 A split ring resonator (SRR) biosensor optimized using FDTD to detect changes in dielectric environment [8]. 
Figure 2.11 Illustration of the invisibility cloak at the molecular level [9]. The incoming incident rays, as a result of the material properties, "bend" around the molecules, making the cloak appear seemingly invisible.....

Figure 2.12 Representation of an electromagnetic field generated by the localized surface plasmons generated at the metal-dielectric interface $[10] \ldots \ldots \ldots 26$

Figure 2.13 A) Illustrates the electromagnetic excitations or collective polarized oscillations of SPPs propagating along the $\mathrm{x}$ direction. $H_{y}$ is the magnetic field that is perpendicular to the propagating waves (shown as coming out of the page). Figure 2.13 B) illustrates the electric field's exponential decay in the metal and the dielectric medium represented respectively by $\delta_{m}$ and $\delta_{d}[11] \ldots \ldots \ldots \ldots \ldots \ldots \ldots \ldots \ldots \ldots \ldots \ldots \ldots \ldots \ldots \ldots$

Figure 2.14 Non-propagating electromagnetic excitations or polarized oscillation surrounding only the isolated metallic nanoparticles $[12] \ldots \ldots \ldots \ldots \ldots$

Figure 2.15 Illustration of an adhesive inter layer to override the compatibility of some noble metals to the dielectric layer. . . . . . . . . . . . 31

Figure 2.16 Representation of a single unit celled electromagnetic metamaterial constructed with a metal-dielectric-metal or "sandwich" scheme. . . . . . 33

Figure 2.17 Schematic representation of Landy et al. [13] origianl structure (a) and the Tuong et al. [14] modified structure (b) . . . . . . . . . . . . . 39

Figure 3.1 a) Schematics of a first order petal shaped fractal nano-antenna. b) Second order petal shaped fractal nano-shaped antenna........... 47

Figure 3.2 Illustration of the periodic boundary condition for the unit-celled

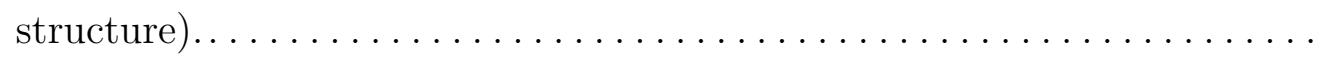

Figure 3.3 The electric and the magnetic components of the waveguide at the inlet

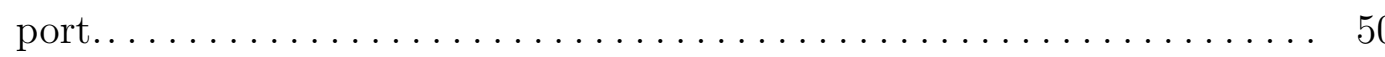

Figure 3.4 Schematics of normalized s-parameters applied in the calculation of

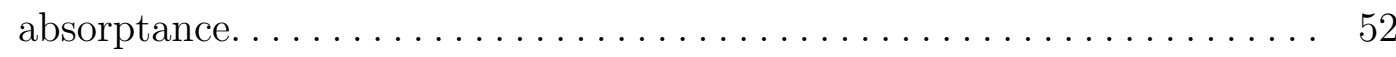


Figure 4.1 Perfect Absorber using a double split-ring resonator and a parallel cut wire at the back to provide individual tuning of electric and magnetic

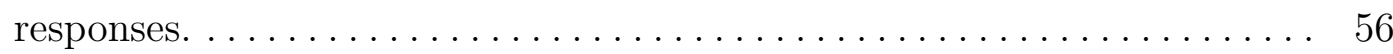

Figure 4.2 Near unity absorption peak in the $\mathrm{GHz}$ region $\omega_{0}=11.2 \mathrm{GHz} \ldots \ldots 56$

Figure 4.3 Validation of the simulated results of the perfect split ring resonator as shown in Figure 4.2 with experimentation data. The dotted gray lines represents the experimental results as obtained by researcher Landy et.

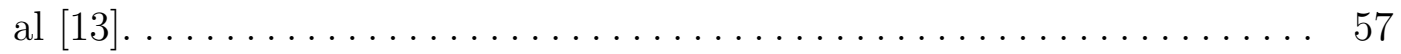

Figure 4.4 First order perfect absorber through the application of fractal design. . . 57

Figure 4.5 Broadband absorber as a result of increasing degree of freedom as a

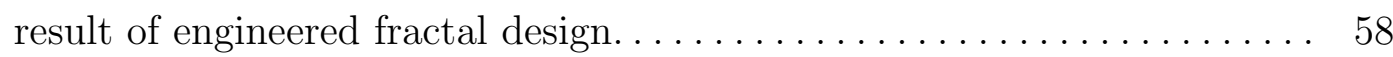

Figure 4.6 A comparison of the bandwidth of the absorption peak in the first order absorber with second order absorber with increasing degree of freedom. . 58

Figure 4.7 a)First order, b) Second order and c) Third Order with increasing order of fractal nano-antennas respectively to create a broadband absorber using the hybridization effect of multi-resonances. . . . . . . . . . .

Figure 4.8 Absorptivity response of the second order absorber (see Figure $4.7 \mathrm{~b}$ ), to illustrate the increase in the number of absorption peaks contributing to a broadband absorber. $\ldots \ldots \ldots \ldots \ldots \ldots \ldots \ldots$

Figure 4.9 Dimensional illustration of the metamaterial nano-antenna constructed from a nanoparticle separated from a ground plane with an alumina dielectric. Critical parameters to this research are the particle width $\left(W_{p}\right)$ and dielectric thickness $\left(T_{D}\right)$. The metamaterial composite is

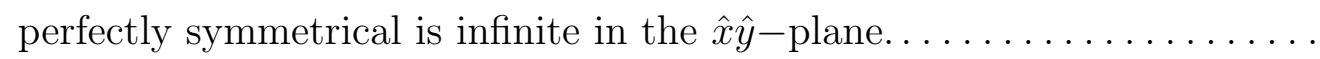

Figure 4.10 Front view illustration of the unit cell nano-antenna utilized as Desgin A containing all the parameters used in the composite construction. ....

Figure 4.11 Absorptivity response of the silver nano-particle antenna of Design A as shown in Figure 4.10 with dimensions $W_{p} \times L_{p} \times T_{p}=50 \mathrm{~nm} \times 50 \mathrm{~nm} \times 30 \mathrm{~nm}$ and a dielectric thickness of $T_{D}=12 n m$ with electromagnetic radiation propagating in the

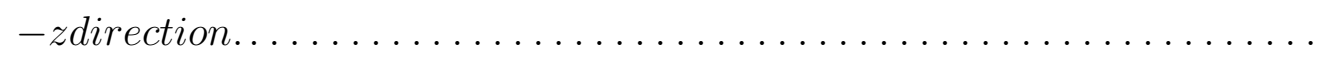


Figure 4.12 At resonant frequency $\omega_{0}=501 \mathrm{THz}$, a) Distribution of the induced (anti-parallel) current. b) Electric field distribution. c) Magnetic Field Distribution. d) Distribution of the the power loss density (absorption). . 64

Figure 4.13 A comparison between different passes in the frequency domain solver of Design A as shown in Figure $4.10 \ldots \ldots \ldots \ldots \ldots \ldots \ldots \ldots$

Figure 4.14 A comparison between TE and TM polarization on the absorption effect of the First Order Absorber of Design A as shown in Figure 4.10. . 67

Figure 4.15 Frequency response of three different unit-celled nano-antennas to observe the influence of geometry on the absorption response. . . . . . . 68

Figure 4.16 The electromagnetic response in terms of absorption for three different unit cells as presented in Figures 4.15 a), b) and c)..............

Figure 4.17 Plot of the normalized absorptivity as a function of both the particle width $\left(\mathrm{W}_{p}\right)$ and the dielectric thickness $\left(T_{d}\right)$. The figure illustrates a bifurcation of the peak absorption with a decrease in frequency. This figure can aid in determining geometric aspects of a $\mathrm{Al}_{2} \mathrm{O}_{3}$ dielectric-silver composite metal materials (see Figure 4.9 for

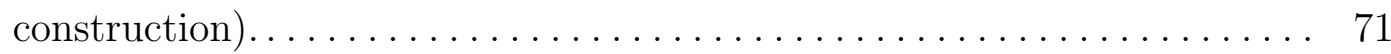

Figure 4.18 Plot of the frequency response versus dielectric thickness for a $\mathrm{W}_{p}=80 \mathrm{~nm}$ particle. Figure (a) is the reflectivity and figure (b) is the absorptivity. There are two peak aborption regions. The first mode is at a dielectric thickness of $25 \mathrm{~nm}$ and the second mode is at a dielectric

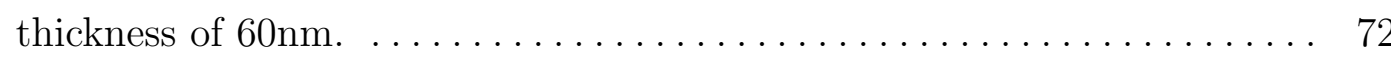

Figure 4.19 Plot of the frequency response versus particle width. The dielectric thickness $\mathrm{T}_{d}=50 \mathrm{~nm}$ and the particle is assumed square. Figure (a) is the reflectivity response and Figure (b) is the absorptivity. Peak absortion is peak at a particle size of $75 \mathrm{~nm} . \ldots \ldots \ldots \ldots \ldots \ldots \ldots \ldots$

Figure 4.20 A two dimensional illustration of the unit cell computational domain and the critical dimensions. Interaction between neighboring particles was handled through symmetry boundary conditions at x-min and $\mathrm{x}$-max. The actual computational domain used in FDTD calculation was $3 \mathrm{D}$ dimension, where the $3 \mathrm{rd}$ dimension is equivalent to the $\mathrm{x}$-dimension. . . . . . . . . . . . . . . . . . . . . 
Figure 4.21 Illustration of Figure 4.20 as an array arrangement to study the interaction effects with changing particle spacing............ 76

Figure 4.22 Illustration of the three dominant interactions that are responsible for the response resulting from an array arrangement. Design B is a cavity interaction between particles. Design $\mathrm{C}$ is an effective medium design.

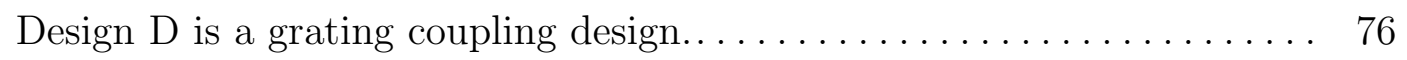

Figure 4.23 Results from Design B of an cavity response between particles resulting in perfect absorption. As the cavity increases the response blue shifts... 78

Figure 4.24 Interaction effects with increasing separation distance between unit cell. The dielectric thickness is held constant at $12 \mathrm{~nm}$ of Design B as shown

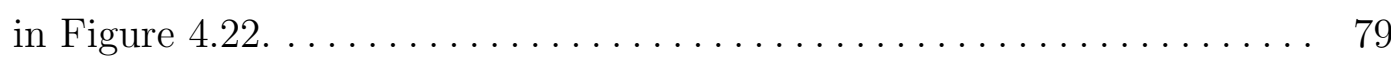

Figure 4.25 Interaction effects with increasing separation distance between unit cell. The dielectric thickness is held constant at 56nm of Design B as shown

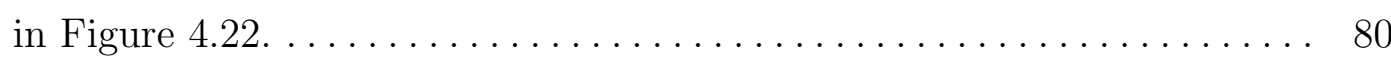

Figure 4.26 Interaction effects with increasing separation distance between unit cell. The dielectric thickness is held constant at 100 of Design B as shown in

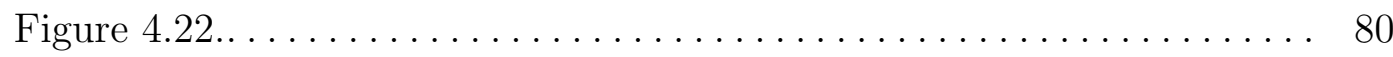

Figure 4.27 Results of Design $\mathrm{C}$ as an effective medium material where the volume fraction of the silver and air impedance match with the surrounding air. . 82

Figure 4.28 Results of Design D of a grating coupler where the peak resonance is apparent when the spacing between the particles is twice the particle

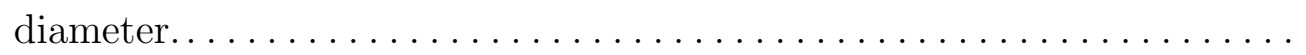

Figure 4.29 Sweep in the electric field direction (L1) to study the effect of electric resonance a) and sweep in the magnetic field direction (W1) to study the effect of the magnetic resonance b).................

Figure 4.30 Combined absorption response of the electric and magnetic resonances. Figure 4.29 a) presents the absorption response by inducing a changing electric field and b) presents the absorption response by inducing a

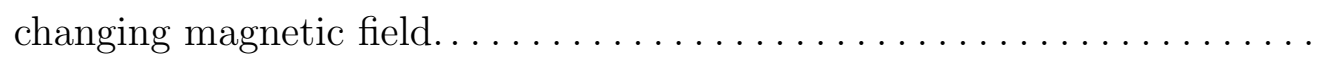

Figure 4.31 Contour representation of the combined absorption response of the electric and magnetic resonances for a particle width $W_{p}=50 \mathrm{~nm}$. . . . . 
Figure 4.32 Contour representation of the combined absorption response of the electric and magnetic resonances for a particle width of $W_{p}=50 \mathrm{~nm}$

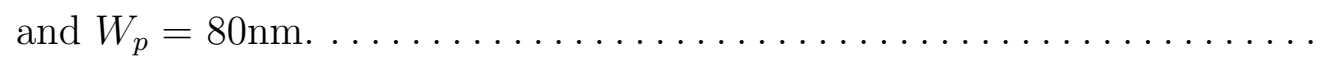

Figure 4.33 Electric and magnetic field distribution for the W1 sweep for resonant frequencies at $\omega_{1^{s t}}=482.5 \mathrm{THz}, \omega_{2^{n d}}=559 \mathrm{THz}, \omega_{3^{\text {rd }}}=658.2 \mathrm{THz}$,

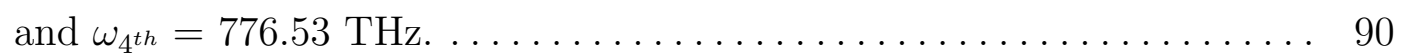

Figure 4.34 Proposed optimized thermal absorber for the unit-celled silver

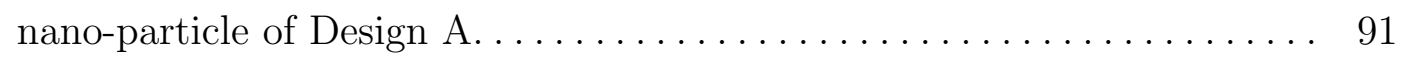

Figure 4.35 Perspective and front view of the optimized second order absorber. . . . 92

Figure 4.36 Contour representation of the absorption response for the second order

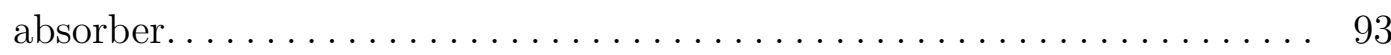

Figure 4.37 Singular resonant peak for the first order absorber. . . . . . . . . 93

Figure 4.38 Multi-resonant peaks for the second order absorber $\ldots \ldots \ldots \ldots \ldots$

Figure 4.39 Perspective view of a third order absorber as an evolution from the first

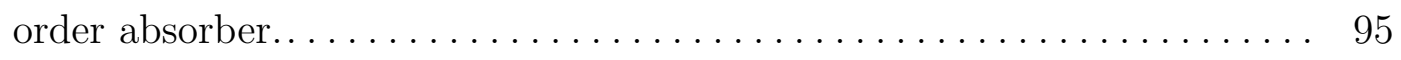

Figure 4.40 A comparison between the absorptivity response of the first order, second order and third order absorber designs. . . . . . . . . . . 96

Figure 5.1 Proposed plasmonic metamaterial thermal absorber construction for

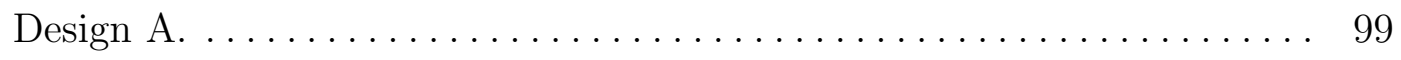




\section{LIST OF TABLES}

Table 2.1 Comparison of material properties of some common noble metals used in the construction of MMs. Information obtained from CST Mircrowave Studio 2012 material's library. . . . . . . . . . . . . . . . . .

Table 3.1 Critical parameters used in the construction of a nano-particle sized unit celled antenna for the First Order Absorber design. . . . . . . . . . . 48

Table 4.1 Critical parameters used in the construction of a nano-particle sized unit celled antenna to study the interaction effects arising as a result of repeating boundary conditions...................... 62

Table 4.2 Dispersion parameters used in the Drude model for the nanoparticle (silver) and the dielectric (alumina).

Table 4.3 Critical parameters used in the construction of a nano-particle sized unit celled antenna to study the interaction effects arising as a result of

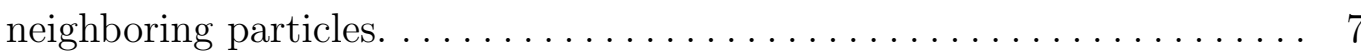

Table 4.4 A comparison of the bandwidth at each resonant modes for successive

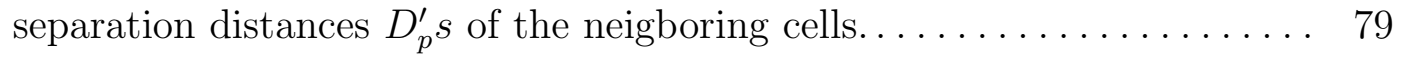

Table 4.5 Critical parameters used for the (L1) sweep to study the effect of the electric resonance for a particle width of $50 \mathrm{~nm}(80 \mathrm{~nm}) \ldots \ldots \ldots \ldots \ldots$

Table 4.6 Critical parameters used for the (W1) sweep to study the effect of the magnetic resonance for a particle width of 50nm $(80 \mathrm{~nm}) \ldots \ldots \ldots \ldots . \ldots 6$ 


\section{LIST OF SYMBOLS}

$l$ Length $(m)$

$\lambda$ Wavelength $(m)$

$E \quad \operatorname{Energy}(J)$

$\nu$ Frequency $\left(\frac{1}{s}\right)$

$h$ Plank's constant $\left(6.626 \times 10^{-34}(J . s)\right)$

$k_{B}$ Boltzmann's constant $\left(1.381 \times 10^{-23}\left(\frac{m^{2} k g}{s K}\right)\right)$

$c$ Speed of light $\left(\frac{m}{s}\right)$

$K$ Temperature (kelvins)

$F \quad$ Spectral irradiance $\left(\frac{w}{m^{3}}\right)$

$H \quad$ Power density $\left(\frac{w}{m^{2}}\right)$

$\phi \quad$ Photon flux $\left(\frac{\text { no.ofphotons }}{\text { seconds } \times m^{2}}\right)$

$\delta$ Declination angle (degrees)

$\alpha \quad$ Elevation angle (degrees)

$\varphi \quad$ Latitude (degrees)

$R$ Radius (degrees) 


\section{CHAPTER 1}

\section{INTRODUCTION}

\section{$1.1 \quad$ Background}

To be able to realize a material with extraordinary exotic properties such as negative index refraction, chirality etc. that surpasses the properties of any natural material, we need more than just a traditional material, we need a Metamaterial or an Electromagnetic Metamterial. Also known as a left-handed material, metamaterials (MMs) can be characterized by two important material parameters that lays the groundwork for its modifications, maneuvering and individual tunability to achieve its numerous applications. As an effective medium [15], these parameters are; a complex electric permitivity, represented by Equation 1.1, and a complex magnetic permeability, represented by Equation 1.2.

$$
\begin{gathered}
\tilde{\epsilon}(\omega)=\epsilon_{1}+i \epsilon_{2} \\
\tilde{\mu}(\omega)=\mu_{1}+i \mu_{2}
\end{gathered}
$$

By a careful and thorough design of the unit cell structure of the macroscopic metamaterial composite, $\epsilon$ and/or $\mu$, can be tailored to exhibit the desired exotic electromagnetic features as mentioned above. Metamaterials make use of surface plasmon polaritons (SPPs) either localized or propagating at the metal-dielectric interface naturally occurring at the interface as a result of metal-dielectric interaction.

These SPPs, with a natural frequency, $\omega_{S P P s}$, are already in oscillatory motion. Upon excitation by an external source such as a light with a driving frequency, $\omega_{d f}$, they increase in vibration. The incoming photons induce a temporary dipole in the oscillating SSPs. Once 
the condition $\omega_{S P P}=\omega_{d f}$ is achieved, resonance/spectral peaks are observed which is where the maximum absorption of heat occurs. These SPPs relax by emitting heat which can be scattered in the external surrounding or absorbed in the constituents of the macroscopic metamaterial composite.

Metamaterials have been shown to work in all the wavelengths of the electromagnetic radiation and has garnered a lot of interest especially in the visible regime for its applicability as a heat generating device.

\subsection{Motivation}

One of the major problems encountered by MMs that has seriously undermined the rapid growth and development of realistic plasmonic metamaterial devices is the optical loss occurring in the traditional metallic constituents, that is, silver, gold etc. of the metamaterial composite. Hence, to develop devices targeted towards heat generating purposes, it is of utmost importance to design, characterize and fabricate new metamaterial devices with high loss in the visible regime. In our research, we are going to focus heavily on creating such a low-loss heat generating device such that the heat absorbed by the plasmonic resonance can easily be confined, controlled, harvested and used for energy generating purposes. In essence, our intention is to create a high-loss nanometer-sized heat source which is able to control optical heating in the visible region. Ultimately we wish to utilize the same unit cell nanometer-sized optical heat controlling device and optimize it in terms of geometry, orientation and topology to create a broadband solar antenna that can be placed on top of a thermoelectric system for solar to electricity direct energy conversion.

\subsection{Problem Statement}

Metamaterials have successfully employed the phenomenon of magnetic resonance uncoupling in the recent years to produce almost perfect absorbers $[6,13,16,17]$. Almost all 
of these designs utilize magnetic excitation of the polariton and perfect impedance matching. While perfect absorbers may posses many alluring applications from super lens to the treatment of cancerous cells, these devices are designed to work at a specific frequency or the resonant frequency of the device. This also implies that the resonant frequency also has a very narrow bandwidth. This prohibits these devices to be utilized for any other purposes except for the one for which it is designed, thus, limiting its practical applications. One of the other disadvantages of these types of resonant plasmonic perfect absorbers is that they are highly sensitive to their design parameters and environment and slight variance in configuration and size can shift the resonance peak making it virtually useless and impractical even though it might be a unity perfect absorber. This limitation can be easily overcome if the the structure is designed with multiple coupled resonators, that is, to exhibit numerous, discrete resonant frequencies at all wavelengths. The hybridization effects of several localized surface plasmons (See Section 2.5.2.2) will then result in an extended continuous frequency band, "multiband" or "broadband" device. The absorbing properties of this device would then be tuned to achieve an absorption of $100 \%$ within a very wide range. As a result, in comparison to the previous perfect metamaterial absorbers achieved from uncoupling magnetic resonance, ours seek to achieve tunable broadband by several magnetically coupled resonator systems. Another dominant problem that has hampered the construction and practical applications of resonant perfect absorbers as well broadband absorbers is the fact the loss occurs in the transmitting medium as well as from the surface of the composite. Yet another problem, opposing the rapid development of perfect broadband absorbers is that some designs are heavily polarization-dependent whereby a slight shift in the angle of incidence renders these designs virtually useless. Moreover, traditional materials such as carbon and its derivatives such as carbon black and graphite have been long considered as good absorbers. The absorptivity of such conventional black absorbers are in the range of 0.8 - 0.85 as shown by Mizuno et al. [18]. The perfect absorbers realized from electromagnetic metamaterials have the capability to achieve absorptivity in excess of 0.95 as will be seen in 
the following chapters.

\subsection{Research Objective}

The objective of our research is two fold. Firstly, to create a broadband absorber by designing a magnetically coupled, resonator system with a response with a continuous frequency band. This would be achieved by benefiting from the hybridization effect of the localized surface plasmons. Moreover, this system will be tuned to attain an abosrption close to $100 \%$ in terms of the geometry, orientation, size of the metallic nanostructure and the dielectric environment. Also to design this broadband absorbers whose components does not rely on frequency, that is, frequency independent, and would be able to absorb radiation over a wide range of bandwidth. The following are the major research objectives:

- To be able to successfully create an ideal but a realizable broadband perfect metamaterial absorber capable of absorbing energy at all wavelengths in the visible regime, that is, from approximately 300nm - 800nm. This broadband perfect metamaterial absorber would then be implemented as a super-solar antenna to achieve absorbance $A(\omega)$ close to $100 \%$.

- To perform computer simulations using finite-difference time domain (FDTD) method using the commercial CST solver to identify and explain the resonant peaks of a first order, unit celled, metamaterial absorber using the appropriate boundary conditions. To use the CST and constitutive relation of the single unit celled metamaterial composite parameters, realize a perfect metamaterial absorber.

- Employing the information gathered from the simulation results of the perfect single unit celled solar absorber and utilizing the geometric scalability of the electromagnetic MMs, progress from the first order absorber to a higher order absorber using fractal design such that the metallic nano structures are able to represent all possible sub-wavelengths in the visible regime. The resonance and polarization of plasmonic 
nanostructures, which offers enhanced light-matter interaction, is often governed by the configuration and proportion of the nanostructure. This is why we propose fractal plasmonic metamaterial on our switch from a first order unit celled perfect absorber to a higher order broadband absorber. The introduction of fractal plasmonic metamaterials has the added advantage in its ability to increase the degree of freedom of the material parameters and provide a solid platform to support multiple resonances, hence, a much more versatile metamaterial. Hence, advance towards the creation of a perfect broadband metamaterial absorber.

- To ensure that our perfect broadband metamterial absorber is polarization-independent so that it could have the same advanced performance at all angles of incoming incidence. This would be particularly useful in the placement of solar cells on top of thermalelectric systems.

- Finally, conduct a study on other possible geometrical unit celled perfect absorbers which can be extended to higher orders to create an ideal broadband perfect metamaterial absorbers while keeping in mind the realizability of such designs considering the fabrication and characterization techniques available at hand.

\subsection{Research Scope}

The scope of this research is to optimize a nano-meter sized particle to, first, create a perfect absorber and secondly, study the interaction effects of "neighboring particles" which arise as a result of repeating boundary conditions. To use the knowledge form the perfect absorber demonstration and interaction effects to create higher order broadband absorbers through the application of fractal design. 


\subsection{Contribution}

In this research, I will be conducting an investigation of higher order absorbers through the utilization of a specific fractal design. This construction of a higher order absorber and it's application as a broadband perfect absorber has not be studied till to date. This fractal design is an evolution from a single square shaped nano-antenna situated on the center of the domain which has been translated diagonally in the four quadrants by an optimal translation vector. This diagonal translation can be carried out to infinity in progression to higher order absorbers. In addition, I would be seeking to achieve a broadband response from this specific fractal design by tuning the geometry of the structure in terms of combination of various parameters. However, before the higher order absorber is given inception, I will look at the behavior of complex interations that arise as a result of neighboring particles. This

will also assist in understanding interactions of higher order absorbers and how it influences absorptivity response in terms of geometry and design, thus, ulitmately influencing our heat generative device. 


\section{CHAPTER 2}

\section{LITERATURE REVIEW}

\subsection{Maximum amount of energy absorbed from the sun}

The light that we see everyday is barely a fraction of the light emitted by the sun incident on the earth's surface. Sunlight is a form of electromagnetic radiation and visible light is a tiny subset of this electromagnetic radiation as illustrated in Figure 2.1.

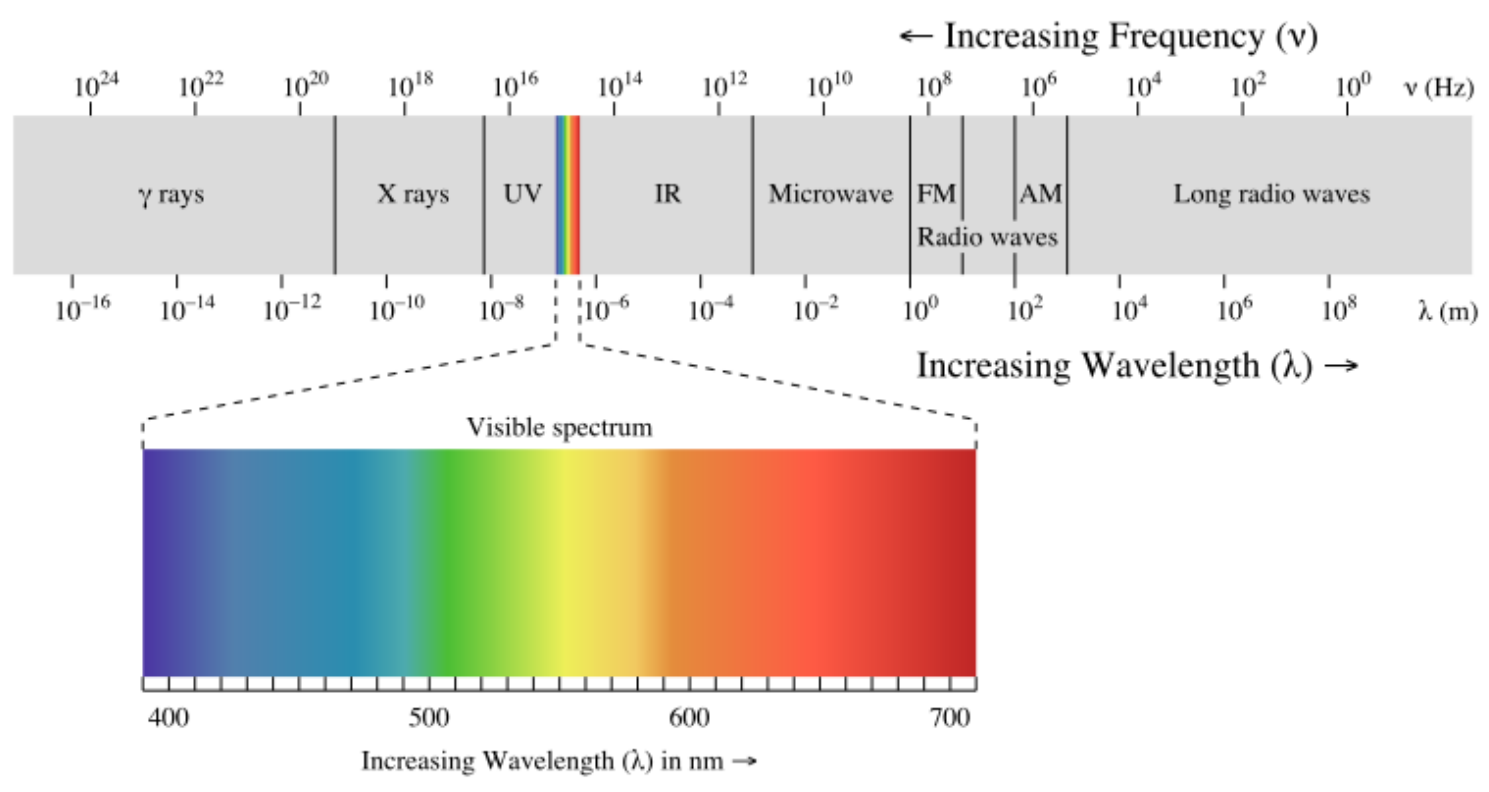

Figure 2.1 A visualization of the visible spectrum as a subset of the electromagnetic radiation [1].

Radiation is one of the fundamental modes of heat transfer. It is distinguished from convection and conduction modes because of its ability to transfer heat energy in the absence or presence of a conducting medium. Radiation take the form of either electromagnetic waves or photons, "a packet of energized particles". Even though radiation can traverse vacuum, it originates from matter [2] undergoing mechanisms such as molecular vibration in gases, 
and electron and lattice vibrations in solids. Thermal radiation is a radiation type in which the temperature of the body or surface directly influences emission, for example, the sun. Electromagnetic radiation (EM radiation or EMR) is a form of energy emitted and absorbed by charged particles which exhibits wave-like behavior as it travels through space. EMR has both electric and magnetic field components, which stand in a fixed ratio of intensity to each other, and which oscillate in phase perpendicular to each other and perpendicular to the direction of energy and wave propagation.

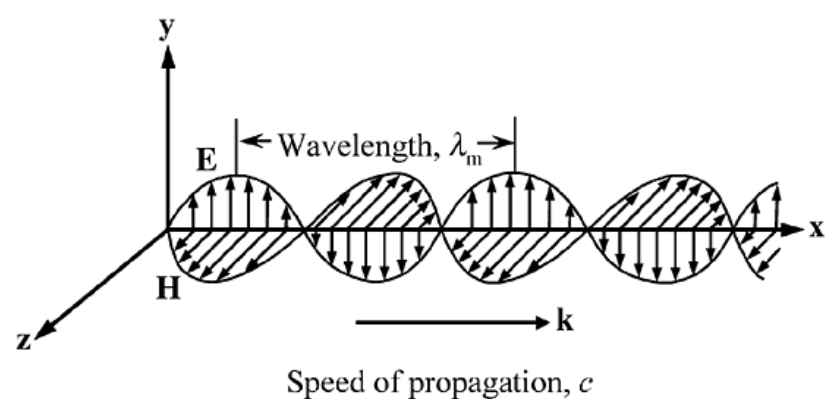

Figure 2.2 The $\bar{E}$ and $\bar{H}$ vectors for a plane electromagnetic wave propagating in the positive direction along the x-axis. The direction of propagation is a result of the cross between the $\bar{E} \times \bar{H}$ vectors. The two vectors are in phase, but perpendicular to each other $[2$, $1]$.

The emergence of quantum-mechanics has been able to explain, the previously barely understood, the dual wave-particle phenomena of the light. Since, visible light, a component of electromagnetic radiation is composed of indistinguishable tiny elements of energy or "quanta of energy", it is of great interest to deduce the amount of energy received on the earth's surface as a result of incident sun's rays. In other words, the solar irradiance or the power density on the earth's surface. The following equations below governs the maximum amount of energy that can be attained on the earth under optimal conditions; 


\subsubsection{Energy of photon}

A photon can be characterized using a wavelength, $\lambda$ or a quantity of energy, $E_{P}$. Since $\nu=\frac{c}{\lambda}, E_{P}$ can be written as,

$$
E_{P}=\frac{h c}{\lambda}=h \nu
$$

where, $h$ is the Plank's constant at $6.626 \times 10^{-34} J . s, c$ is the speed of light at $2.298 \times 10^{8} \frac{\mathrm{m}}{\mathrm{s}}$, $\lambda$ is the particular wavelength in $m$ and $\nu$ is the frequency of the particular wavelength in $s^{-1}$.

\subsubsection{Photon Flux}

The photon flux, $\phi$, is defined as the number of electrons per second per unit area.

$$
\phi=\frac{\text { no.ofphotons }}{\sec \times m^{2}}
$$

\subsubsection{Power Density}

The photon flux is important as it assists in calculating the power density, $H$ by multiplying the energy of a single photon.

$$
H\left(\frac{w}{m^{2}}\right)=\phi \times \frac{h c}{\lambda}=\phi E_{P}
$$

\subsubsection{Spectral Irradiance}

The spectral irradiance, $F$, is a useful way of characterizing any given light source as it provides the power density at any given wavelength,

$$
F(\lambda)=\phi E \frac{1}{\lambda}
$$


where, $F(\lambda)$ is in $\frac{w}{m^{3}}$ and $\phi$ is in $\frac{\text { no.ofphotons }}{\text { sec } \times m^{2}}$.

\subsubsection{Radiant Power Density}

The total power density emitted from a light source can be calculated by integrating the spectral irradiance over all wavelengths $[2,4]$.

$$
H=\int_{0}^{\infty} F(\lambda) d \lambda=\int_{0}^{\infty} \frac{\phi E_{P}}{\lambda} d \lambda=\sum_{i=0}^{N} F_{i} \triangle \lambda_{i}
$$

At this juncture, it can be surmised that to calculate the total amount of power absorbed by the earth's surface, it is important to calculate the radiant power density of the sun. In order to perform this calculation, first the spectral irradiance of the sun has to be calculated. It is a well known fact that the sun behaves like a "blackbody" emitter, hence, blackbody conditions will be applied to calculate its spectral irradiance as shown in the succeeding equations.

\subsubsection{Sun's Spectral Irradiance modeled as a Blackbody Radiator}

According to Bose-Einstein [2], the total number of photons need not be conserved as it depends on temperature, therefore,

$$
\frac{d N}{V}=\frac{d g}{V} \frac{1}{e^{\frac{h \nu}{k_{B} T}}-1} .
$$

In the phase space, the quantum states consist of a volume $V$ and in the momentum space, a spherical shell [2] which is given by,

$$
d g=\frac{8 \pi V \nu^{2} d \nu}{c^{3}}
$$

Given the speed of light $c$ in the medium and the density of states as $D(\nu)=\frac{d g}{V}[2]$, the number of photons per unit volume per unit frequency interval can be written as, 


$$
f(\nu)=\frac{1}{V} \frac{d N}{d \nu}=f_{B E}(\nu) D(\nu)=\frac{8 \pi \nu^{2}}{c^{3}\left(e^{\frac{h \nu}{k_{B} T}}-1\right)} .
$$

Each photon has an energy $E_{P}$ as given by Equation 2.1, therefore the spectral energy density can be written as [2],

$$
u_{\nu}=f(\nu) E(\nu)=\frac{8 \pi h \nu^{3}}{c^{3}\left(e^{\frac{h \nu}{k^{T}}}-1\right)} .
$$

For a given surface area, the relation between the radiant energy flux to the energy density and the speed of light is given by [2], where $\frac{c}{4}$ is a geometrical factor.

$$
F_{r a d, \nu}=\frac{u_{\nu} c}{4}
$$

After substituting Eguation 2.9 into Equation 2.10 we obtain,

$$
F(\nu, T)=\frac{2 \pi h \nu^{3}}{c^{2}\left(e^{\frac{h \nu}{k_{B} T}}-1\right)} .
$$

After substituting the expression $\nu=\frac{c}{\lambda}$ into Equation 2.11, Equation 2.12 is obtained which is the spectral irradiance of a blackbody or Plank's Distribution of a blackbody, hence, the spectral irradiance of the sun,

$$
F(\lambda, T)=\frac{2 \pi h c^{2}}{\lambda^{5}\left(e^{\frac{h c}{k_{B} \lambda T}}-1\right)},
$$

where, $T$ is the temperature of the blackbody in kelvins, $K$, and $k_{B}$ is the Boltzmann constant at $1.381 \times 10^{-23} \frac{\mathrm{m}^{2} \mathrm{~kg}}{\mathrm{sK}}$.

Substituting all the values of the constants, and the temperature of sun taken to be $6000 K$, the sun's spectral irradiance is obtained. Figure 2.3 illustrates the sun's spectral irradiance distribution as well as Planck's blackbody irradiance [3]. 


$$
F_{\text {sun }}(\lambda)=\frac{3.744 \times 10^{-16}}{\lambda^{5}\left(e^{\frac{2.402 \times 10^{-6}}{\lambda}}-1\right)}
$$

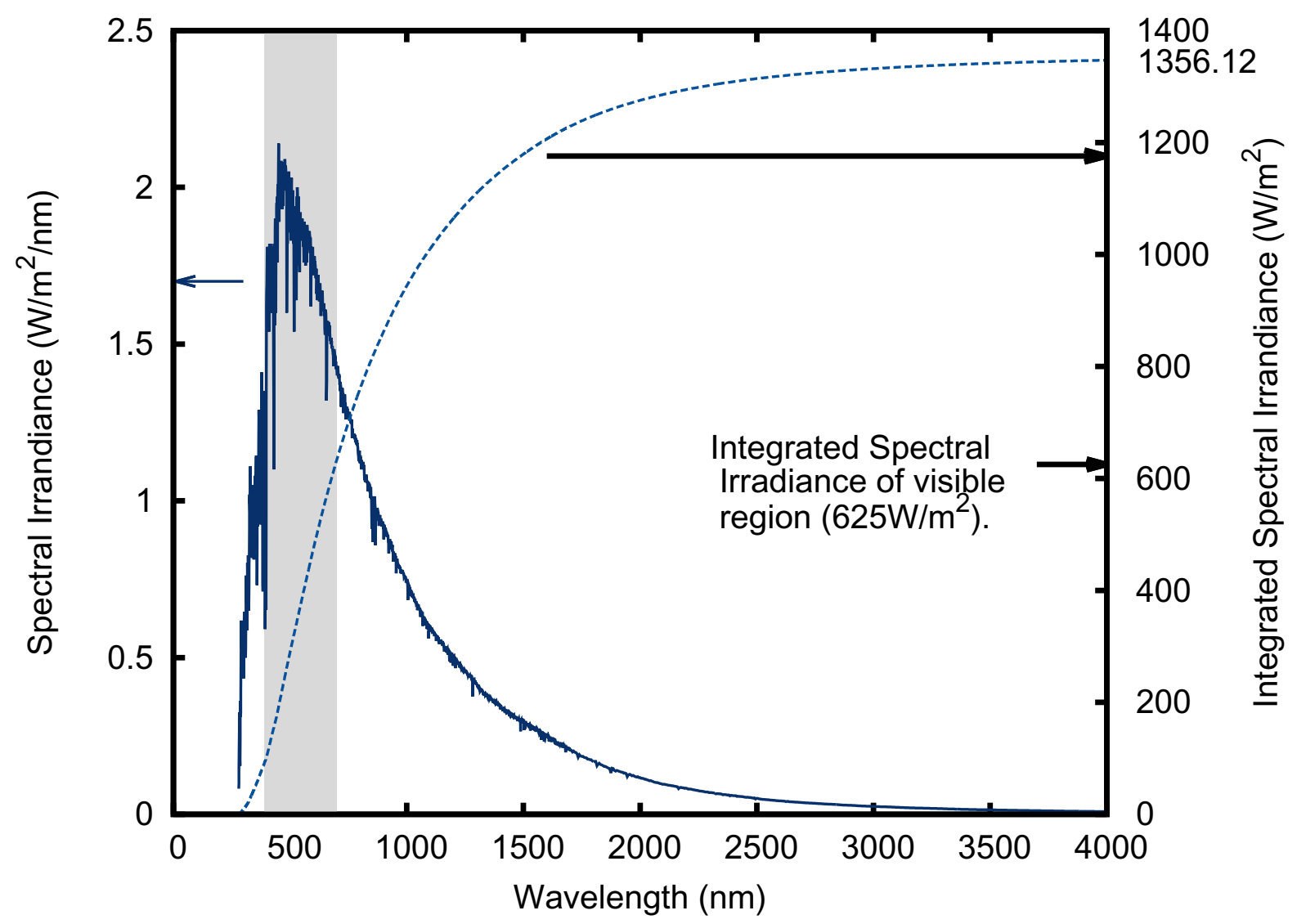

Figure 2.3 Illustration of solar spectral irradiance as a function of wavelength regenerated from data obtained from National Institute of Standards and Technology [3].

Utilizing Figure 2.3, the total amount of solar power reaching the earth's surface was calculated. This was achieved by integrating the total area under the spectral irradiance versus wavelength curve for a value of $1356.12 \frac{\mathrm{W}}{\mathrm{m}^{2}}$. Afterwards, the total area under the curve for the visible region (shaded region) was calculated for a value of $625 \frac{\mathrm{W}}{\mathrm{m}^{2}}$. Comparing these two values it can be seen that the contribution of solar power by the visible region to 
the total solar power reaching the earth's surface is approximately 50\%. Hence, our intense interest to create a perfect broadband absorber in the visible regime.

\subsubsection{Total Solar Radiant Power Density}

Using Equation 2.5, an exact value for solar radiant power density is obtained which is approximate to the value in literature [4].

$$
\begin{aligned}
H_{\text {sun }} & =\int_{0}^{\infty} F_{\text {sun }}(\lambda) d \lambda \\
& =\int_{0}^{\infty} \frac{3.744 \times 10^{-16}}{\lambda^{5}\left(e^{\frac{2.402 \times 10^{-6}}{\lambda}}-1\right)} d \lambda \\
& =62.5 \frac{M w}{m^{2}}
\end{aligned}
$$

\subsubsection{Solar Radiation in Space}

As mentioned in Section 2.1, an object in outer space is recipient of a fraction of the total power emitted from the sun. $H_{s}$ is the power density incident on an object due to illumination from the sun. At a distance $D$ from the sun, the $H_{s}$ can be calculated as follows,

$$
H_{s}=\frac{R_{\text {sun }}^{2}}{D^{2}} \times H_{\text {sun }}
$$

Given that the outer atmosphere of the earth is at a distance of $1.500 \times 10^{11} \mathrm{~m}$ as shown in Figure 2.4, the maximum amount of energy that can be absorbed by the earth's atmosphere from the sun or the solar irradiance on earth's atmosphere $H_{\text {earth }}$ would be [4], 


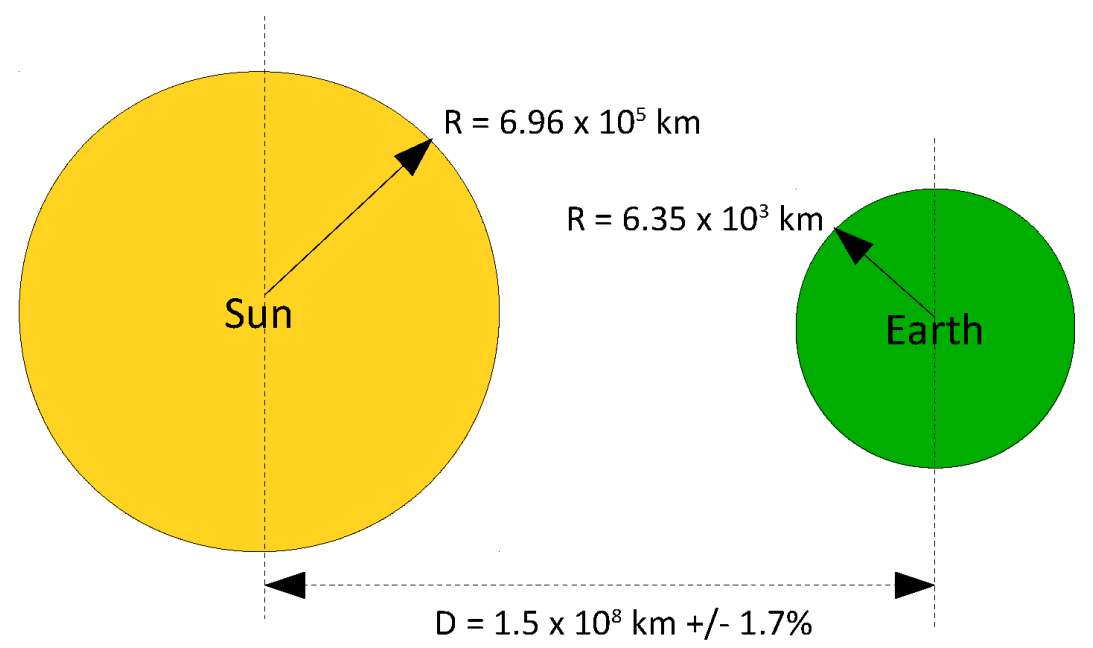

Figure 2.4 Relative position of the earth from the sun and their specific dimensions [4].

$$
\begin{aligned}
H_{\text {earth }} & =\frac{R_{\text {sun }}^{2}}{D_{\text {earth }}^{2}} \times H_{\text {sun }} \\
& =\frac{\left(6.955 \times 10^{8}\right)^{2}}{\left(1.496 \times 10^{11}\right)^{2}} \times 62499432.6 \\
& =1345.49 \frac{\mathrm{w}}{\mathrm{m}^{2}}
\end{aligned}
$$

At this point in time, the value calculated above will suffice. This is because, as will be seen in the subsequent chapters, our plasmonic fractal metamaterial models or plasmonic devices (PDs) closely resemble the sun-earth perpendicular relation in vacuum. That is, the energy from our theoretical EMR source is reaching the PDs at an angle of incidence of 90 degrees simulated in vacuum.

The solar irradiance outside the earth's atmosphere is fairly constant throughout the year. However, the actual solar irradiance that reaches the earth's surface is undesirably less. One of the principal reasons for this reduction is the atmospheric effect which includes absorption, scattering and reflection in the atmosphere, water vapor, clouds, pollution etc. On the other hand, it is well known that the earth revolves around the sun as well as on its own axis which is titled at $23.45^{\circ}$. Therefore, the other factors which prompt this reduction are the fixed location on the earth's surface which is given by the longitude and the latitude, the time of day and the day of the year. As can be visualized in Figure 2.4, given minimal 
atmospheric effects, the maximum amount of solar irradiance that can reach the earths surface is when the sun is perpendicular to the earth. Hence, during the design of a solar array, the second set of factors can be considered such that the solar array will always be tilted perpendicular to the sun. This requires a very precise tracking of the sun's position and can achieved by considering the declination angle, the elevation angle, the zenith angle and the azimuth of the sun position relative to a fixed position on earth as described in detail by researchers [19], [20], [21] and [22].

\subsubsection{Tracking the Sun's Position}

\subsubsection{Declination Angle}

The declination of the sun is the angle between the equator and a line drawn from the centre of the Earth to the centre of the sun [23] The declination angle $\delta$, is a result of earth's tilted axis of $23.45^{\circ}$ which other wise would always be $0^{\circ}$. The equation below shows how the declination angle can be calculated.

$$
\delta=23.45^{\circ} \sin \left[\frac{360}{365}(d-81)\right]
$$

Where, $\mathrm{d}$ is the day of the year with Jan 1 as $d=1$.

\subsubsection{Elevation Angle}

The elevation angle is the angular height of the sun in the sky measured from the horizontal. The elevation is $0^{\circ}$ at sunrise and $90^{\circ}$ when the sun is directly overhead.

An important parameter in the design of photovoltaic systems is the maximum elevation angle [23], that is, the maximum height of the sun in the sky at a particular time of year. The maximum elevation angle is at solar noon and varies with latitude and declination angle. The equation below shows how the elevation angle can be calculated. 


$$
\alpha=90-\varphi+\delta
$$

Where, $\varphi$ is the latitude of interest and $\delta$ is the declination angle calculated by Equation 2.17.

\subsubsection{Azimuth Angle}

The azimuth angle is the compass direction from which the sunlight is coming from. At solar noon, the sun is always directly south in the northern hemisphere and directly north in the southern hemisphere. At the equinoxes, the sun rises directly east and sets directly west regardless of the latitude, thus making the azimuth angles $90^{\circ}$ at sunrise and $270^{\circ}$ at

sunset. Equation 2.19 shows how the azimuth of the sun can be calculated relating the declination and the elevation angle [20], [19] and [22].

$$
\text { Azimuth }=\cos ^{-1}\left[\frac{\sin \delta \cos \phi-\cos \delta \sin \phi \cos (h r a)}{\cos \alpha}\right]
$$

Where, hra is defined as follows:

$$
\begin{aligned}
h r a & =15^{\circ}(\text { lst }-12), \\
l s t & =l t+\frac{t c}{60}, \\
t c & =4(\text { Longitude }- \text { lstm })+e o t, \\
l s t m & =15^{\circ} . \triangle T_{\text {gmt }}, \\
e o t & =9.87 \sin (2 B)-7.53 \cos (B)-1.5 \sin (2 B), \\
B & =\frac{360}{365}(d-81) .
\end{aligned}
$$

and $l t$ is the local time, lst is the local solar time, lstm is the local standard time meridian and $\triangle T_{g m t}$ is the difference between the local time and the Greenwich Mean Time in hours. 


\subsection{Optical Properties of Metal Nanostructures}

- The interaction of light with metallic nanostrucutures, though previously not as fully understood, was already in use centuries ago [24]. The optical properties of metallic nanostructures were extensively used by artists before we finally begun to fully understand, analyze, appreciate and technologically explore the interaction of light in metallic nanostructures [5]. These properties were employed by them to generate and combine brilliant colors in artefacts and artworks whereby tiny gold particles of varying size and dimensions were imbedded in glass to generate a wide array of alluring colors. Some of the most notable examples are the Lycurgus cup of the Roman Empire and windows of St. Stephans Church in Mainz as show in Figure 2.6. Figure 2.5 is another portrayal of enticing effects achieved by artists by employing the phenomenon of light and metallic nanostructures.

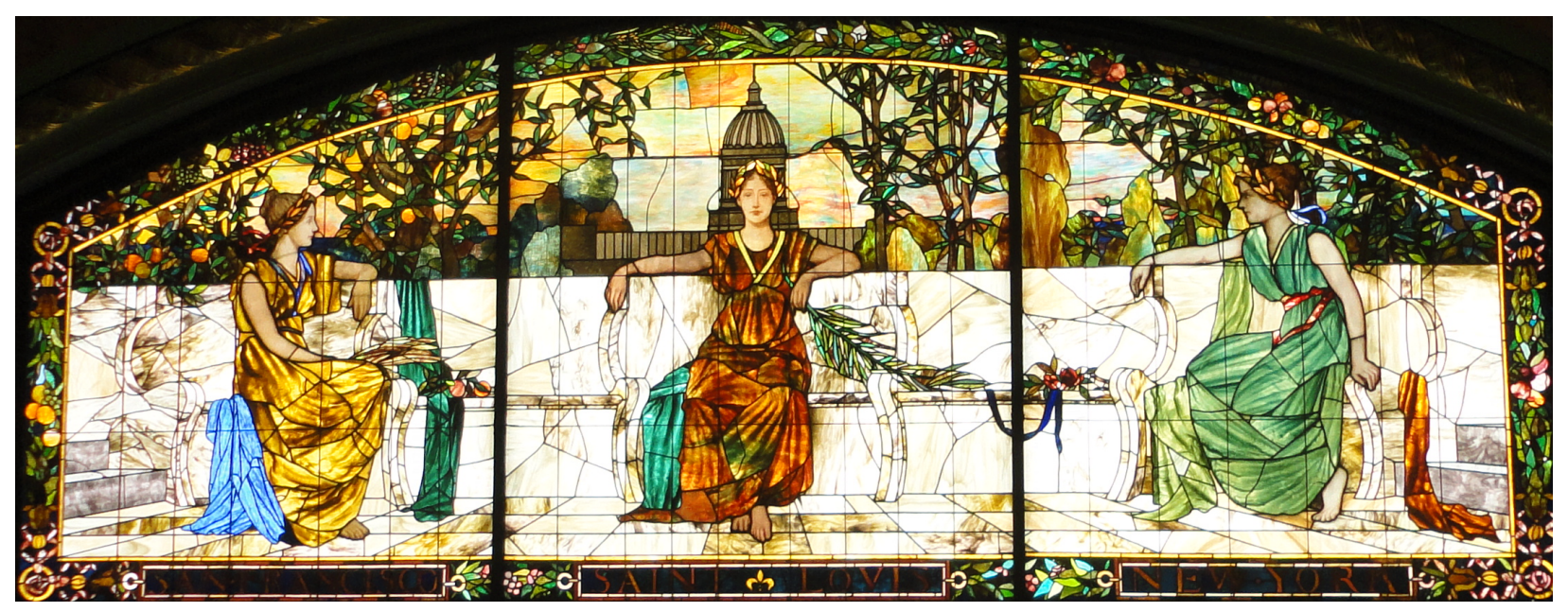

Figure 2.5 Illustration of light and nano-metallic structures interaction taken at the train station mall in Saint Loius Missouri, 2011. 

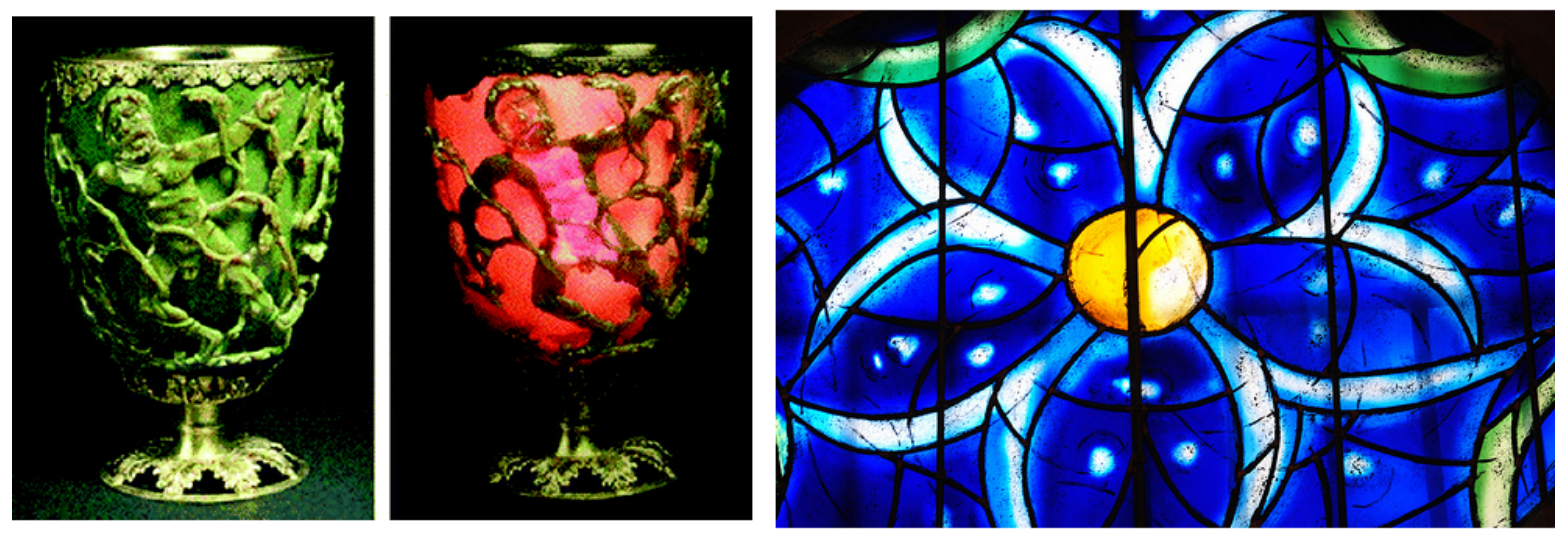

Figure 2.6 Historical illustrations of light-metal interactions: Lycurgus cup shown as green while reflecting light and green while transmitting light and the church window glasses in Mainz [5].

\subsection{Emergence of Metamaterials (MMs)}

The nascent concept of metamaterials, originally known as left-handed material (LHM) or negative refractive index material (NIM), was first put into motion by Veselago in 1968 [6, $25,26]$ because of its ability to possess both negative electric permittivity and negative magnetic permeability at the same time. Due to lack of experimental verification, the concept of metamaterials lay dormant until it was further revolutionized by Sir Pendry [9] upon his discovery of a wire medium which was characterized by negative permittivity and permeability.

A metamaterial is an artificially tailored macroscopic composite composed of either periodic, as shown in Figure $2.7 \mathrm{~A}$ ) or non-periodic structure as shown in Figure $2.7 \mathrm{~B}$ ). Their peculiar but exotic and extraordinary properties stem from both the unit or cellular structure and the chemical composition rather than from the characteristics of the individual constituents.

MMs are classified into two groups: the resonant absorbers and the non-resonant type. The biggest advantage of the resonant MMs is that it has a broader change rate for the electric permittivity, $\epsilon$, and magnetic permeability, $\mu$, as presented in Figure 2.8 with a 
small change in the resonant frequency [6]. Figure 2.8 shows the classification of all materials that can be accounted in nature. The x-axis represents, $\epsilon$, which is the absolute value of the real and imaginary part of the electric permittivity as shown in Equation 2.21. The y-axis represents, $\mu$, which is the absolute value of the real and imaginary part of the magnetic permeability as shown in Equation 2.23. The first quadrant represents all the materials that can be found in nature such as wood and carbon. The free space of air is the location on this axes where $\epsilon=\mu=1$. The intersection of the two axis represents non-existence whereby no materials can be found in the physical environment, that is, $\epsilon=\mu=0$. The second and the fourth quadrant represents materials that cannot be physical created in a laboratory setting. The third quadrant is the position where the metamaterials lie, hence the name, left-handed material (LHM) or negative index of refraction (NIR). From Figure 2.8, it can be noticed that it is possible to modify $\epsilon$ by modifying the geometry of the metamaterial composite at sub-wavelength scales provided that $\mu$ is known and kept constant.

$$
\begin{gathered}
\epsilon=\epsilon_{1}+i \epsilon_{2} \\
|\epsilon|=\sqrt{\epsilon_{1}^{2}+\epsilon_{2}^{2}} \\
\mu=\mu_{1}+i \mu_{2} \\
|\mu|=\sqrt{\mu_{1}^{2}+\mu_{2}^{2}}
\end{gathered}
$$

Hence, a wide variety of material parameters can be achieved by controlling the particle's resonant frequency and vice versa. Landy et al. [13] demonstrated an almost perfect resonant absorber MMs by using primarily metallic structures and likewise Tuong et al. [27] was able to achieve an almost perfect resonant absorber using a symmetrical, four 
petaled, flower shaped structure.

Both these examples serve to show the versatility and the flexibility of MMs to manipulate resonance peak by changing material parameters and vice. While non-resonant MMs also have resonant frequencies, they being extremely large make them less desirable.
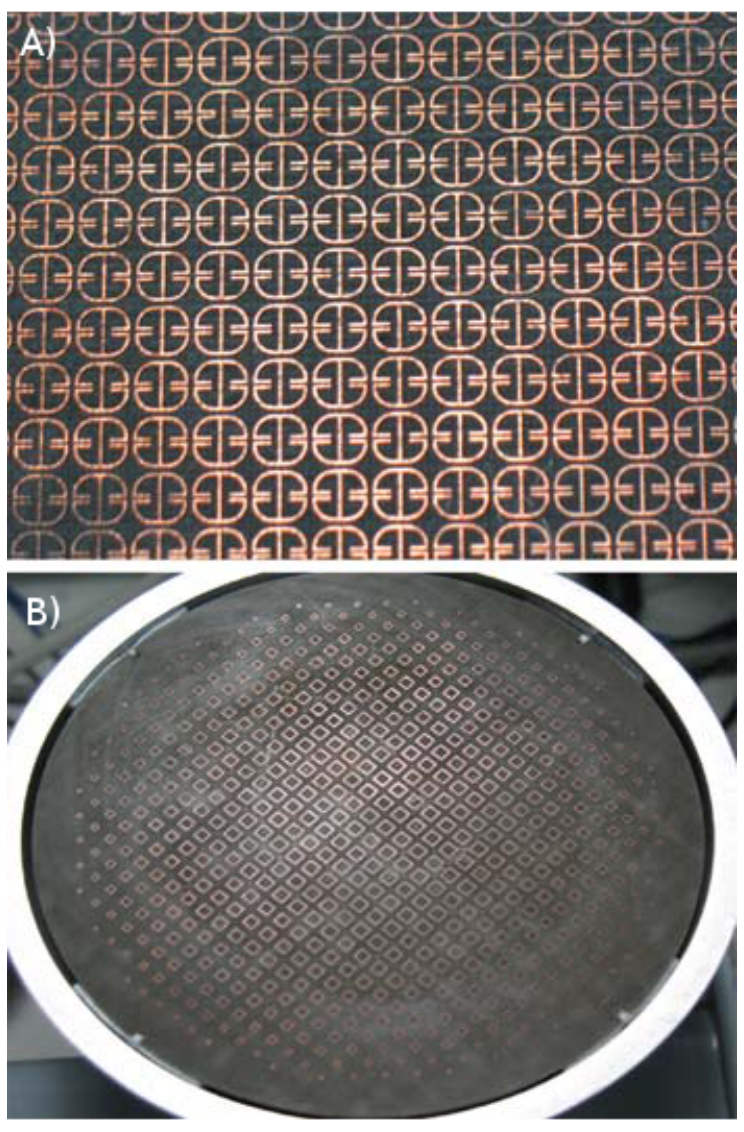

Figure 2.7 A) Depicts a periodic arrangement of a metamaterial composite in comparison to Figure $2.7 \mathrm{~B}$ ) which depicts a non-periodic arrangement metamaterial composite [6]. 


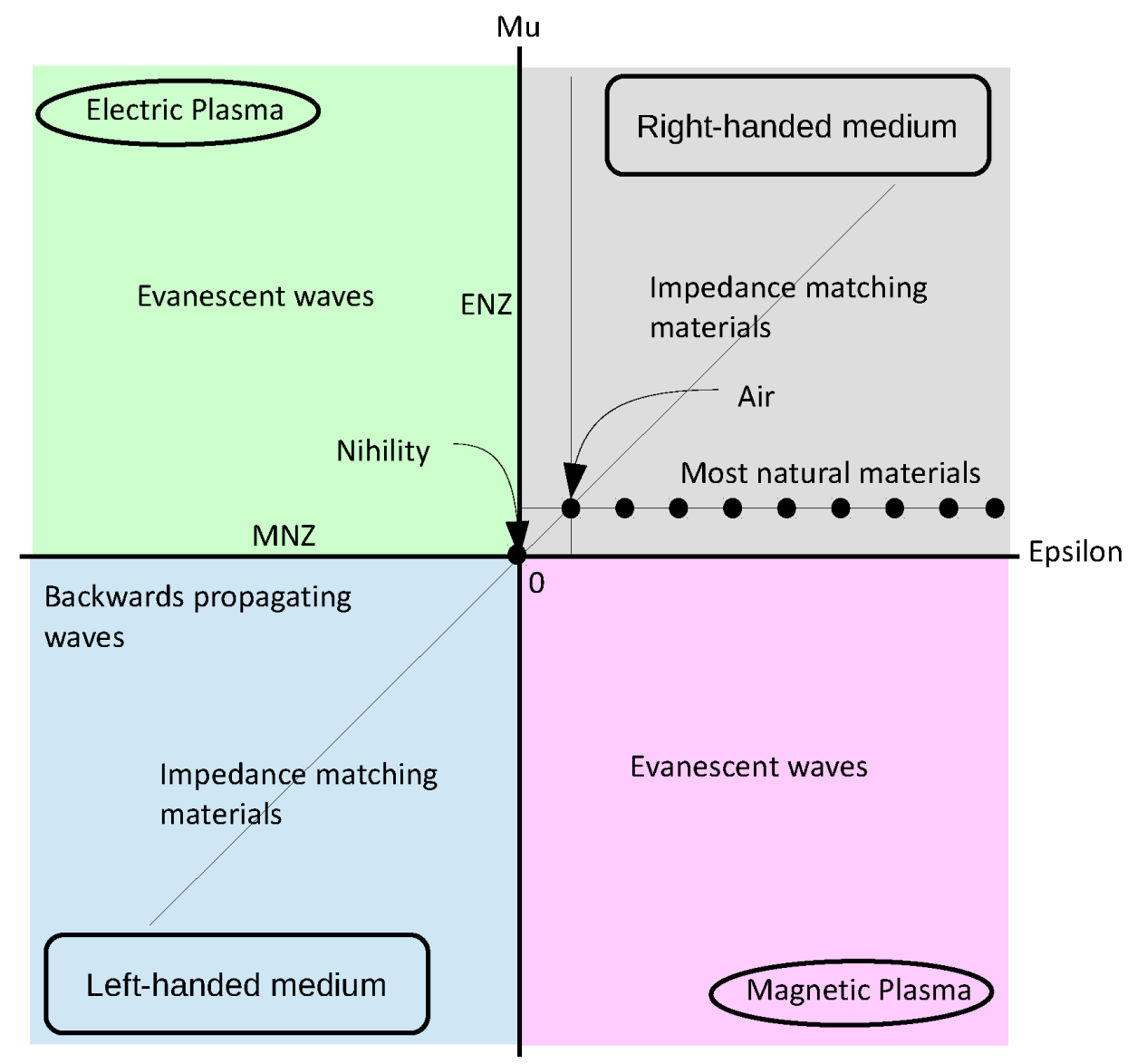

Figure 2.8 A comparison of typical materials found in nature in relation to Left-handed materials [6].

\subsection{Applications of Metamaterials}

\subsubsection{Superlens}

One of the most attractive applications that has resulted through the emergence of metamaterials or left-handed materials are superlens as shown in the Figure 2.9. The arrangement of this superlens is periodic as discussed in Section 2.3. A superlens is a metamaterial whose constituents can both attain its electric perimitvity, $\epsilon$, and magnetic permeability, $\mu$, as -1 . As a result of its extremely super high resolution [28], it is gaining rapid popularity in medical imaging, optical imaging and non-destructive detections. Cui et al. [6] suggests that the first superlens was optimized to work in the visible and infrared 
regime and was later improved to provide resolution three times greater than the traditional setback involving diffraction limit. Superlens operate by exciting the magnetic and the electric fields and coupling the surface modes to the near fields of a target on one side and bringing the image to a focus on the opposite side [7]. This is of great assistance as electricity and magnetism are usually nearly decoupled in the near field. The most recent development has been achieved by researcher Guy [7] who through the utilization of a square slab, has been able to demonstrate a superlens which utilizes long range wireless power transfer, thus, minimizing, the health hazards by high frequency magnetic fields.

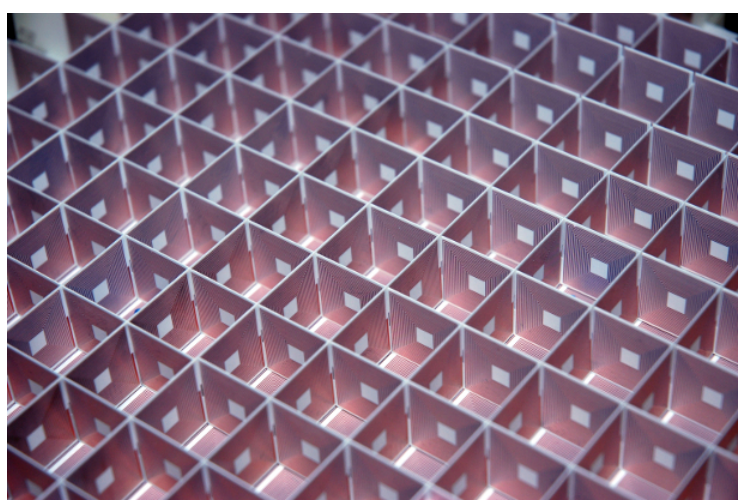

Figure 2.9 A cuboidal shaped super high resolution superlens operating using wireless power transfer [7] and hence, detect cancerous cells.

\subsubsection{Biosensor and Cellulor Detector}

Metamaterials can be used in treating cancerous cells through the design of a "parasitic cell" composed of a dielectric core, a metallic outer shell and a penetration depth equivalent to the skin thickness of a human cell. By taking advantage of the fact that the refractive index of MMs are very sensitive to changes in their dielectric environment and tunability of resonant frequency through periodic metallic nano-structures scalability, these parasitic cells can bound to cancerous cells selectively by shifting their resonance frequencies relative to the dielectric medium [8]. This would be the result of changing dielectric medium 
which in this case would be blood. Once these cells have attached themselves to the undesired human cells, they can be excited at their particular resonant plasmon frequency for the specific wavelength, for example, through a laser beam, and eradicating the cancerous cells by generating heat in the cell. Shiraga et al. [8] utilized once of the basic metamaterial design, a split ring resonator (SRR), upon which live cancerous cells were cultured in a medium comparable to the human blood. She studied the changes in the reflection of the SRR relative to the changing dielectric medium. As a result, she was able to design a highly sensitive, whole cell probation enabled, novel cellular sensor. A similar research has been conducted by Willets et al. [12] whereby she utilized shift in resonance frequency of the localized surface plasmon as a spectroscopic and sensing device. She implemented this by employing the plasmon's spectral location and its sensitivity to the local environment to detect which the diagnosis of "any disease with an associated biomarker and antibody pair, for example, ovarian cancer.

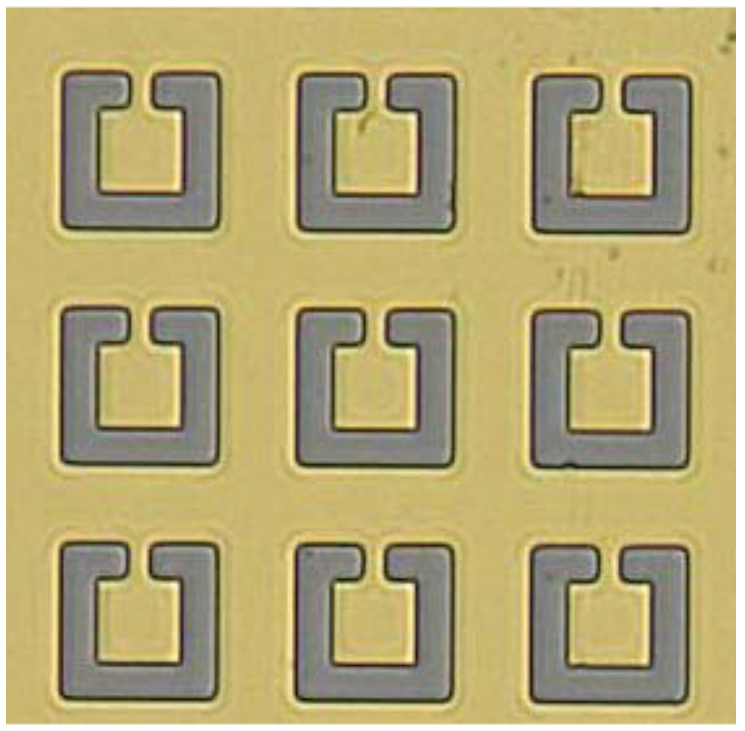

Figure 2.10 A split ring resonator (SRR) biosensor optimized using FDTD to detect changes in dielectric environment [8]. 


\subsubsection{Invisible Cloaks}

Pendry et al. [9] was amongst the first to introduce the concept of an invisibility cloak as depicted in the Figure 2.11. Henceforth, it has been studied in detail by many researchers $[24,15,29]$ in various ways with peculiar geometery such as polygonal cloak, elliptical cloak, cuboidal cloak [30] and so forth. The invisibility cloak has been designed by tempering the traditional conservative quantities of electromagnetism, that is, electric

displacement field, $\vec{D}$, the magnetic field intensity, $\vec{B}$, and the Poynting vector, $\vec{S}$ such that these fields can be made to avoid an object and flow around them unhindered and returning to the original path. This design was further optimized by researcher Shengying et al. [31] who studied the dispersion effect of the invisibility cloak using the the simplified Drude dispersion model which until then was not studied.
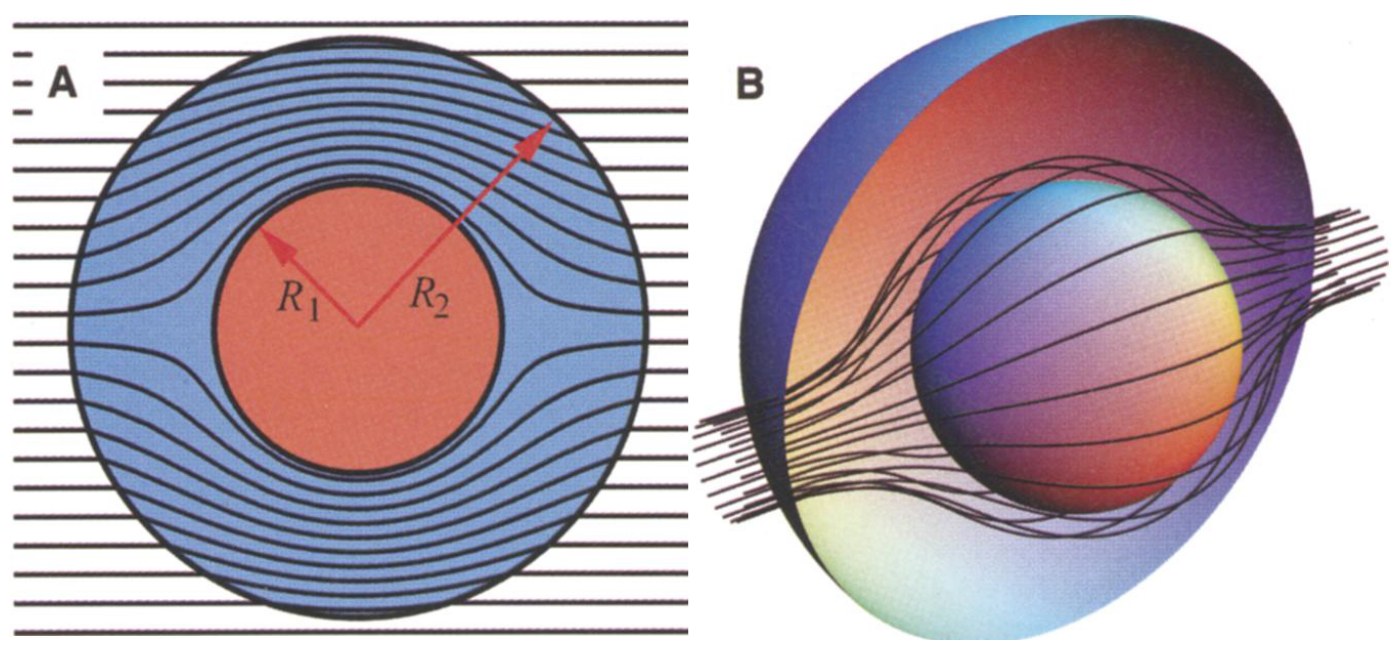

Figure 2.11 Illustration of the invisibility cloak at the molecular level [9]. The incoming incident rays, as a result of the material properties, "bend" around the molecules, making the cloak appear seemingly invisible. 


\subsection{Construction of Electromagnetic Metamaterials}

\subsubsection{Electromagnetic Wave Absorption Theory}

Upon incident electromagnetic radiation on a given material's surface [32, 33, 34], the various ways in which these waves intersect with medium is through the following; reflectance, R $(\omega)$, transmittance, T $(\omega)$, absorbance, A $(\omega)$, scattering or excitation of the surface electromagnetic waves induced by the surface plasmon polaritons (see Section 2.5.2). All electromagnetic metamaterials designs rely on the above mentioned properties which grants easy access for electromagnetic radiation to propagate through the boundary. Since noble metals such as lossy silver, gold, chromium etc. and dielectrics are such they are being extensively used in the construction. However, in the real design, the actual choice of the materials depend on the particular function that the MM is proposed to serve. For example, resonant MMs is required in the design of a cloak [6] whereas in the design of an invisible cloak, non-resonant MMs are deployed.

\subsubsection{Surface Plasmons}

Surface plasmons are electromagnetic excitations that are present between two adjacent layers of a metal-dielectric composite in the presence of an incoming electromagnetic radiation. These excitations create polarized oscillations at the metal-dielectric interface as illustrated in Figure 2.13. These oscillations have an associated electromagnetic field as shown in Figure 2.12 [10]. As a result, a complete metal-dielectric composite system is obtained in which the metallic constituent has an oscillating electron cloud and the dielectric medium experiencing an electromagnetic wave. These effects fades away exponentially in the respective medium. Hence, a more accurate term for the surface plasmons is coined as surface plasmon polaritons (SPPs). In the dielectric environment, the penetration depth is

approximately $\frac{\lambda}{2}$ of the incident wavelength whereas in the metallic environment it is determined by skin depth $[11,12]$. As a result SPPs can be visualized as an unique case of surface 
waves. The term surface plasmon can be simultaneously used as polarized oscillations of metallic nanostructures alongside electromagnetic excitations [5]. SPPs can either be classified as propagating surface plasmons (PSPs) or localized surface plasmons (LSPs). The term plasmonics is synthesised to explain the relation between metals, their electromagnetic response to light and optical phenomena.

\subsubsection{Propagating Surface Plasmons}

If the SPPs propagate along the metal and dielectric interface $[11,12]$, as the name suggests, they are classified as propagating surface plasmons as presented in Figure 2.12 and Figure 2.13 These waves decay exponentially away from the interface into both the metal and the dielectric constituents as illustrated in Figure 2.13 B). Since the decay length of the dielectric component $\delta_{d}$ is different from the decay length of the metal $\delta_{m}$, as expected, the behavior of the propagating surface plasmons is sensitive to changes in the dielectric environment.

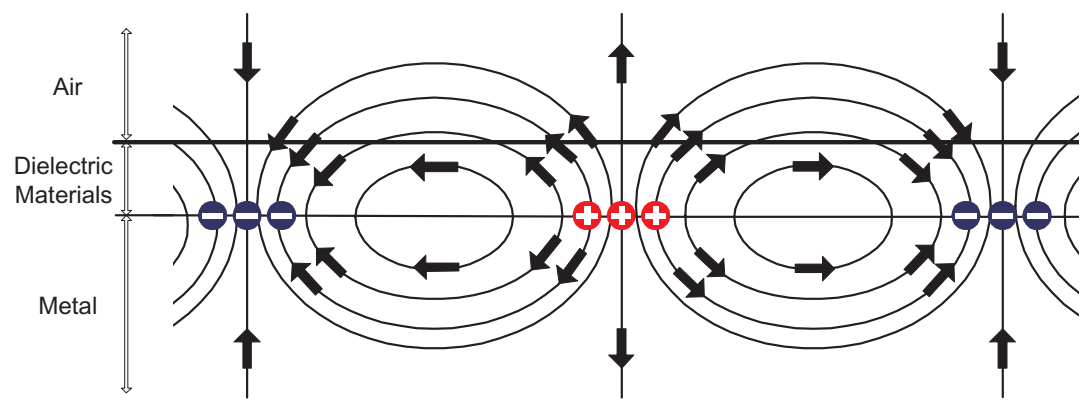

Figure 2.12 Representation of an electromagnetic field generated by the localized surface plasmons generated at the metal-dielectric interface [10]. 
a)

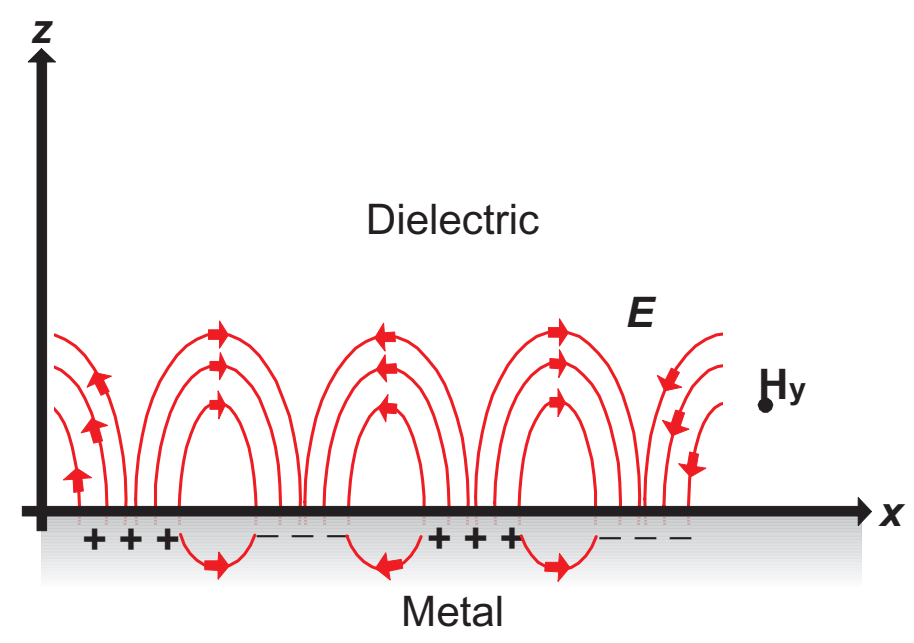

b)

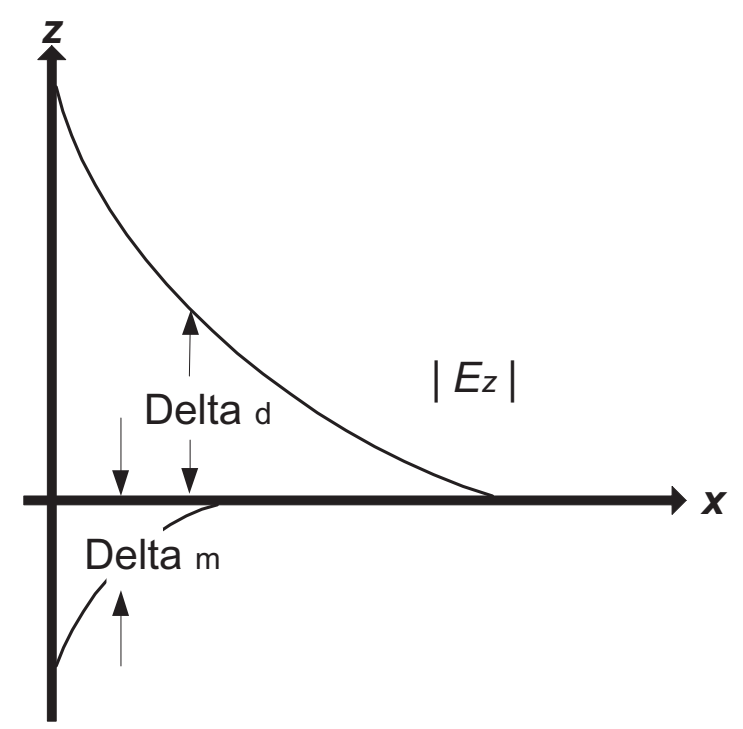

Figure 2.13 A) Illustrates the electromagnetic excitations or collective polarized oscillations of SPPs propagating along the $\mathrm{x}$ direction. $H_{y}$ is the magnetic field that is perpendicular to the propagating waves (shown as coming out of the page). Figure $2.13 \mathrm{~B}$ ) illustrates the electric field's exponential decay in the metal and the dielectric medium represented respectively by $\delta_{m}$ and $\delta_{d}[11]$.

\subsubsection{Localized Surface Plasmons}

Localized surface plasmons (LSPs) are non-propagating electromagnetic excitations that surrounds the metallic nanostructures. For this case, the incident light interact with nano-metallic structures that are much smaller than the incident wavelength. This results in polarizated oscillations that only occurs around the particular nanoparticle. This phenomenon is shown in Figure 2.14 whereby the the blue spheres represent the nanoparticles. In addition, LSPs are sensitive to the changes in the dielectric medium. 


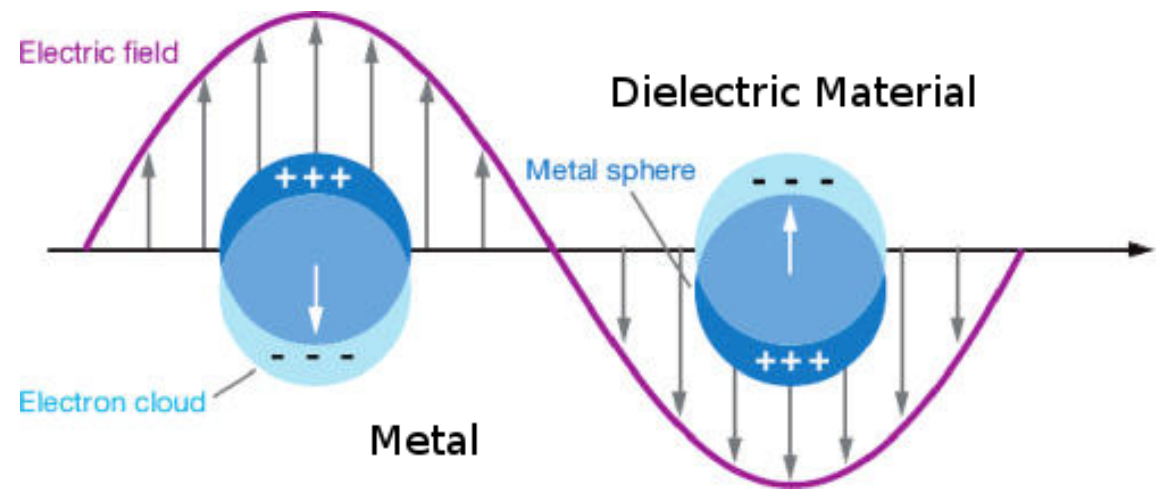

Figure 2.14 Non-propagating electromagnetic excitations or polarized oscillation surrounding only the isolated metallic nanoparticles [12].

\subsubsection{Noble Metals}

The optical properties of noble metals have been discussed by many authors [35, 36, $5,6]$ but for our purposed, two fundamental properties of metals especially noble metals are mentioned;

- The free electrons are able to pass through the bulk material without any restraint in any given direction since noble metals are isotropic homogenous materials

- If the energy of a particular photon is greater than the energy of the band gap for the given metal, interband excitations will occur.

This means that in the presence of an electromagnetic field as shown in Figure 2.12 [37], the electrons are displaced which results in a temporary dipole moment whose relation is represented by Equation 2.24, where $g$ is the unit charge and $\vec{r}$ is the displacement vector for the unit charge. The total dipole moments of all the electrons in the bulk material can result in macroscopic polarization.

$$
\triangle \vec{p}=q \triangle \vec{r}
$$




\subsubsection{Drude Model}

One of the most common models that is used to describe "free electrons" behavior of common realistic materials such as metals and dielectic medium is the Drude model [38]. Almost all metamaterials are composed of some sort of resonant particles such as free electrons which are displaced as a result of incoming radiation. Since, in the construction of our metamaterial devices, gold, silver, alumina $\left(\mathrm{Al}_{2} \mathrm{O}_{3}\right)$ etc. materials are employed, whose interactions with the electromagnetic radiation is determined by the free electrons, a thorough study of the Drude-Model [35] is in order to understand the oscillating particles. The simple Drude model states that free electrons oscillate $180^{\circ}$ out of phase relative to the driving electric field which is why most metals posses a negative dielectric constant[36]. Considering only effects of the free electrons, the relation is shown in the Equation $2.25[36,39,16]$

$$
m_{e} \frac{\partial^{2} \vec{r}}{\partial t^{2}}+m_{e} \gamma_{e} \frac{\partial \vec{r}}{\partial t}=e E_{0} e^{-i \omega t}
$$

whereby $e$ is the charge, $m_{e}$ effective mass of the free electrons, $\vec{r}$ is the displacement vector of the free electrons $E_{0}$ is the electric field's amplitude and $\omega$ is the working frequency and $\gamma$ is the damping constant of the electrons. Solving the Equation 2.25 using $\vec{r}(t)=r_{0} e^{-i \omega t}$ and the relation, $\epsilon=1+\frac{|\vec{P}|}{\epsilon_{0}|\vec{E}|}$ The simple drude model can be written as follows;

$$
\begin{aligned}
\epsilon_{\text {Drude }}(\omega) & =1-\frac{\omega_{p}^{2}}{\omega^{2}+i \gamma \omega} \\
& =1-\frac{\omega_{p}^{2}}{\omega^{2}+\gamma^{2}}+i \frac{\gamma \omega_{p}^{2}}{\omega\left(\omega^{2}+\gamma^{2}\right)}
\end{aligned}
$$

where $\sqrt{\frac{n e^{2}}{m_{e} \epsilon_{0}}}$ the volume plasma frequency and $n$ is the total number of individual dipole moments. Table 2.1 shows the properties of some noble metals used in the construction of MMs in the visible regime using data gathered from the CST microwave 2012. 


\begin{tabular}{|c|c|c|c|}
\hline \hline Noble Metals & $\omega_{\infty}\left(s^{-1}\right)$ & $\omega_{p}\left(s^{-1}\right)$ & $\gamma\left(s^{-1}\right)$ \\
\hline \hline $\mathrm{Ag}$ & 6.0 & $1.5 \times 10^{16}$ & $7.73 \times 10^{13}$ \\
\hline $\mathrm{Au}$ & 10 & $1.37 \times 10^{16}$ & $1.1 \times 10^{14}$ \\
\hline $\mathrm{Cr}$ & 3.2 & $3.5 \times 10^{15}$ & $6.05 \times 10^{14}$ \\
\hline $\mathrm{Al}$ & 1 & $2.4 \times 10^{16}$ & $1.1 \times 10^{15}$ \\
\hline
\end{tabular}

Table 2.1 Comparison of material properties of some common noble metals used in the construction of MMs. Information obtained from CST Mircrowave Studio 2012 material's library.

\subsubsection{Effective Medium Theory}

An electromagnetic metamaterial is usually composed of metallic nanostructures that are some fraction of the incident wavelength, sub-wavelength tructures. Despite being minute in nature when compared to the actual wavelength, they are still able to posses their own dielectric identity. The composite composed of metallic nanostructures, destroys the isotropic homogeneity of the macroscopic material and provides uneven terrain creating overlapping or screening charges. This makes the calculation of individual polarization (see Equation 2.24) of a given dipole an impossible task. The local fields, changed by the uneven charges, exhibit irregular fields. Under certain condition [40, 41, 37] a second average can be calculated which represents the composite as a dielectric function $\varepsilon$. The result of this second average is known as the Effective Medium Theory (EMT). Therefore, EMT, is a more convenient way to characterize the polarization of the macroscopic composite. One of the common procedures is to obtain this average by applying an external electric field, $\vec{E}$, and then solve the Laplace's equation for the microscopic field as a function of geometry and the individual dielectric constituents. The average field is typically applied by capacitor plates so that the following $\vec{E}\left(\vec{r}^{\prime}\right)$ along with its corresponding displacement field, $\vec{D}\left(\vec{r}^{\prime}\right)$ is achieved and then $\varepsilon$ obtained from the relation $\vec{D}=\varepsilon \vec{E}$. However, this second average for the EMT is not without limitations which has been discussed in length by researcher Aspnes [37]. In a detailed study by Koschny et al. [40], the EMT was successfully utilized to study metamaterial structures such as cut wires and split ring resonators of periodic arrangement to study transmission 
and reflectance to determine the effective $\epsilon$ and $\mu$.

\subsubsection{Alternatives to Noble Metals}

One of the major setbacks of traditional building blocks of metamaterials such as gold and silver is that the construction of such sub-wavelength metallic nanostructures, for the Effective Medium Theory to be placed effectively placed in action, undermines the manufacturing and fabrication process. In addition, the optical properties of noble metals like $\mathrm{Au}$ and $\mathrm{Ag}$ are not tunable [42] and cannot be scaled to fit the simulated metamaterial design. Furthermore, noble metals also refuse to be easily embedded in the dielectric media and a secondary compatible media is needed such as chromium as shown in Figure 2.15.

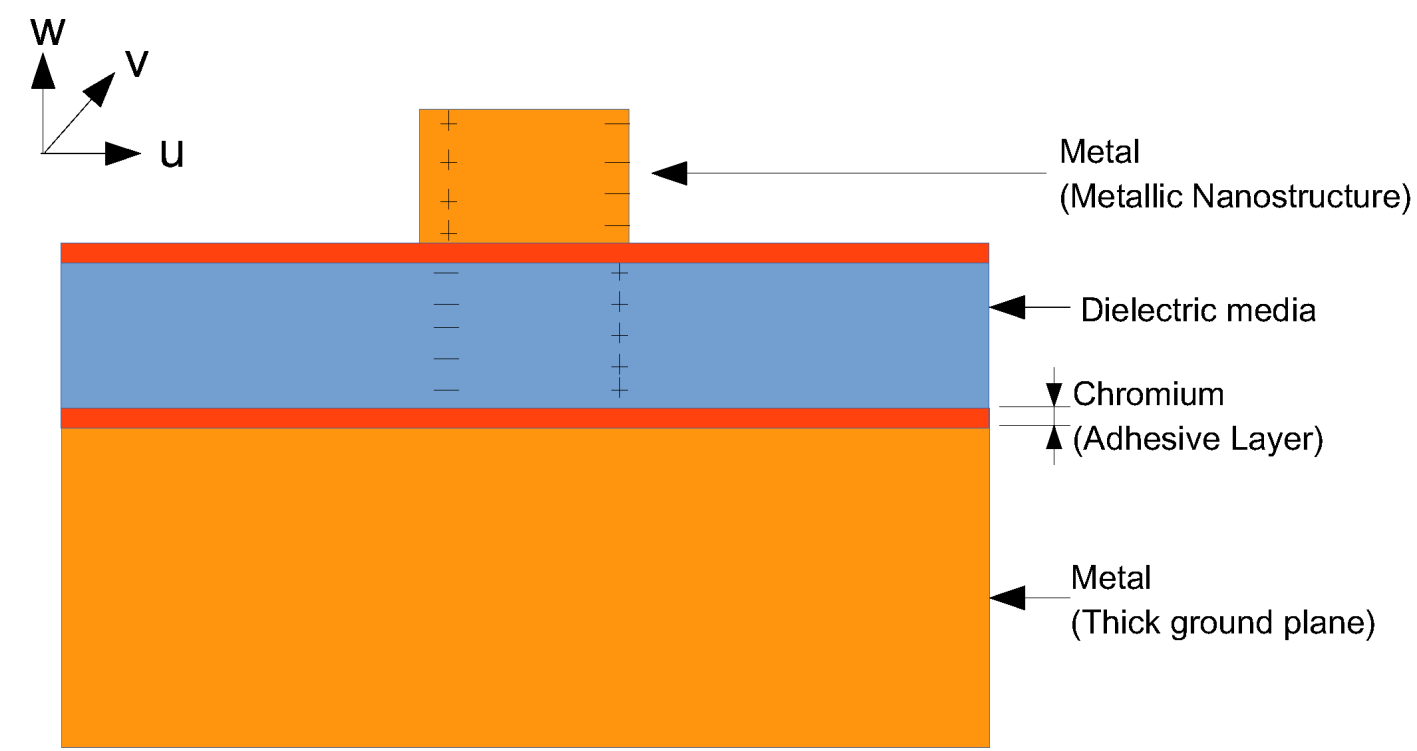

Figure 2.15 Illustration of an adhesive inter layer to override the compatibility of some noble metals to the dielectric layer

As a result, researchers have started to focus on alternatives to noble metals. Researchers Naik et al. [42] have proposed $A l: Z n O$ as a replacement for the metallic constituent for a plasmonic metamaterial construction. They replaced gold with a heavily doped 
$A l: Z n O$ and were able to characterize a nonresonant MMs with a very low absorbance. The MM created with this substitute showed all the typical responses and tunability of a traditional metamaterial absorber while boasting the ease in fabrication.

\subsubsection{Influence of dielectric environment}

The other component of an electromagnetic metamaterial design is the dielectric media, therefore, it is important to understand the effect of this component on the desired responses. Usually MMs are constructed with a three layer design of metal-dielectric-metal sandwich model as shown in the Figure 2.16. From Figure 2.13, it can be seen that the electric field's exponential decay, $\delta_{d}$, depends on the dielectric microscopic material properties. In addition, the $\delta_{d}$ is also affected by the macroscopic property, that is, the dielectric thickness. Hence, it is established that in the design and optimization of plasmonic broadband absorbers, the dielectric media is a crucial parameter. In a study to investigate the effect of the dielectric spacer on the absorptive responses, Li et al. [15] created a dual-band metamaterial absorber consisting of two different elements in the the same unit cell structure. He discovered that the dielectric spacer thickness, when varied from $0.3 \mathrm{~mm}-0.8 \mathrm{~mm}$, exhibited very tiny effects as compared to the effects created by the two metallic elements. However, there was a very tiny blueshift the absorption peaks as a well tiny improvemen. In another study conducted by Drachev et al. [43], the surface roughness of the dielectric media was analyzed by fabricating different grating samples with various degrees of roughness. His results, as expected, showed that the roughness increased losses at the plasmonic resonances which was induced by the increased in the frequency of the electrons collision rate as a result of uneven terrain. Surprisingly, the roughness did not affect the permittivity of the dielectric media. 


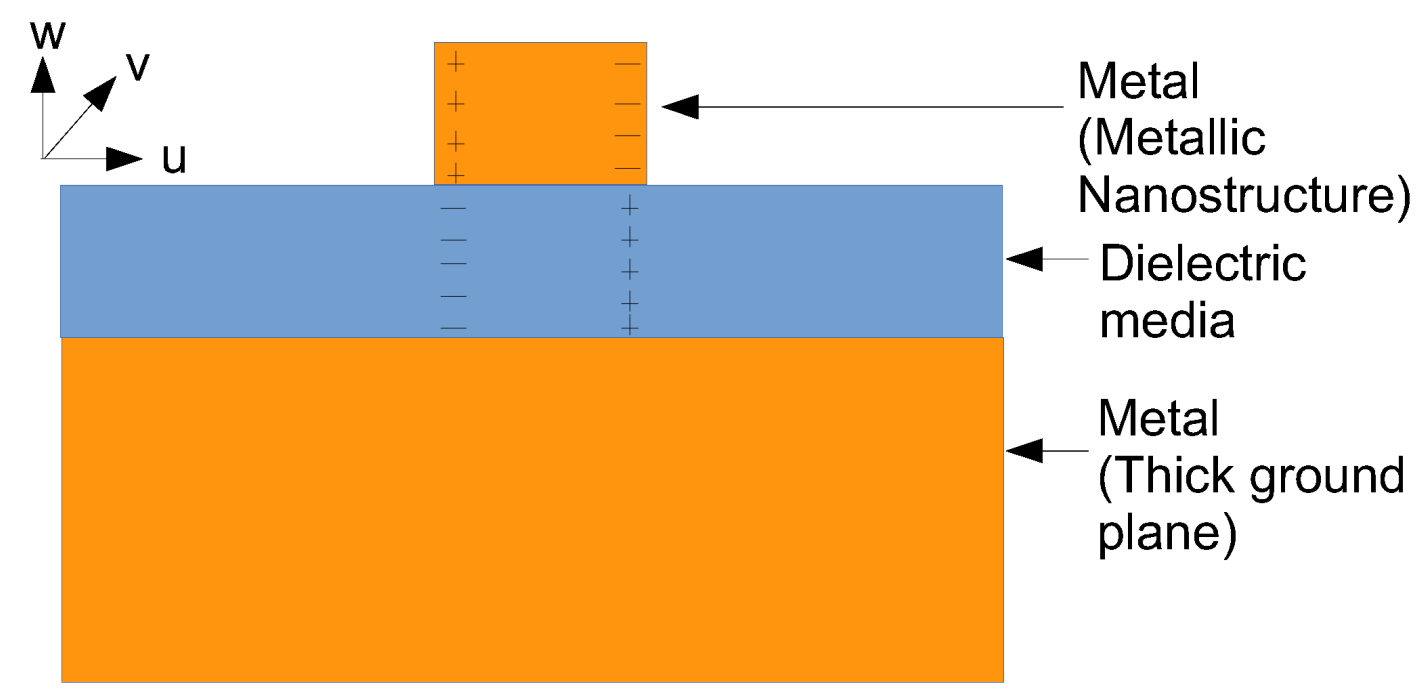

Figure 2.16 Representation of a single unit celled electromagnetic metamaterial constructed with a metal-dielectric-metal or "sandwich" scheme.

\subsection{Absorptive and dispersive Materials}

As shown in Figure 2.12, the existence of an electromagnetic field at the metaldielectric interface implies that there also exists an electromagnetic energy density in the medium which is both electrically and magnetically dispersive and absorptive. Since our study is concerned with the confinement and harvesting of energy from such a medium [44], that is, gold and dielectric, it is of much interest to study their dispersive and absorptive behavior From a classic point of view, in the presence of an oscillating electric field, the particles in the system will act as harmonic oscillators. More accurately, the particles in the system can be treated as damped harmonic oscillators. Assuming the material is isotropic, therefore the restoring force is the same in all direction, a simple relationship for the induced dipole moment, $P$, can be written as follows,

$$
\frac{\partial^{2} \vec{P}}{\partial t^{2}}+\gamma_{e} \frac{\partial \vec{P}}{\partial t}+\omega_{e o}^{2} \vec{P}=\epsilon_{0} \omega_{e p}^{2} \vec{E}
$$

whereby, $\gamma_{e}$ is the electronic damping constant, $\omega_{e o}$ is the resonance frequency and $\omega_{e p}$ is the electric plasma frequency describing the relation between the oscillators and the electric 
field. $\vec{P}$ is the polarization dipole moment, $\vec{E}$ is the electric field and $\epsilon_{0}$ is the dielectric of the free space. For a time dependence harmonic excitation field, the permittivity can be written as shown in Equation 2.28.

$$
\epsilon(\omega)=1-\frac{\omega_{e p}^{2}}{\omega^{2}-\omega_{e 0}^{2}+i \gamma_{e} \omega}
$$

A similar equation can be written for an oscillating magnetic field the induced magnetic moment, $M$, takes the following form,

$$
\frac{\partial^{2} \vec{M}}{\partial t^{2}}+\gamma_{m} \frac{\partial \vec{M}}{\partial t}+\omega_{m o}^{2} \vec{P}=\mu_{0} \omega_{m p}^{2} \vec{H}
$$

whereby, $\gamma_{m}$ is the magnetic damping constant, $\omega_{m o}$ is the magnetic oscillators' resonance frequency and $\omega_{m p}$ is the magnetic plasma frequency describing the relation between the oscillators and the magnetic field. $\vec{H}$ is the magnetic field and $\mu_{0}$ is the magnetic permeability of the medium. Similar to the permittivity's time dependence harmonic field excitation, the time dependence harmonic excitation field for the permeability can be written as shown in Equation 2.30 .

$$
\mu(\omega)=1-\frac{\omega_{m p}^{2}}{\omega^{2}-\omega_{m 0}^{2}+i \gamma_{m} \omega}
$$

Because this research was interested in the absorptivity of the electromagnetic energy, the construction of a Poynting vector $[44,39]$ provides quantitative measure of the energy of the incident wave. 


\subsubsection{Pyonting Theorem}

The Poynting vector is the conservation of electromagnetic energy over a given surface and can be written as the following,

$$
\vec{S}=\int_{\sigma}(\vec{E} \times \vec{H}) d \vec{\sigma} .
$$

\subsubsection{Conservation of Energy}

Using Equations 2.27 and 2.29, Equation 2.32 can be be rewritten in the form of an energy density,

$$
-\int_{v} \frac{\partial u}{\partial t} d V=\int_{V}\left(\frac{\gamma_{e}}{\epsilon_{0} \omega_{e p}^{2}}\left|\frac{\partial \vec{P}}{\partial t}\right|^{2}+\frac{\mu_{0} \gamma_{m}}{\omega_{m p}^{2}}\left|\frac{\partial \vec{M}}{\partial t}\right|^{2}\right) d V .
$$

The calculation of $u$ will yield the total energy density. The physical meaning of the Equation 2.32 represents the energy flow through the boundary surfaces of the volume $V$ per unit time.

From Equations $2.27-2.30$, the rate of energy loss by the absorptive dissipation in the volume. $[45,44,16]$ is written as follows;

$$
\begin{aligned}
q & =q e+q_{m} \\
& =\left\langle\frac{\gamma_{e}}{\epsilon_{0} \omega_{e p}^{2}}\left|\frac{\partial \vec{P}}{\partial t}\right|^{2}+\frac{\mu_{0} \gamma_{m}}{\omega_{m p}^{2}}\left|\frac{\partial \vec{M}}{\partial t}\right|^{2}\right\rangle \\
& =\frac{1}{2} \epsilon_{0} \omega \operatorname{Im} \epsilon(\omega)|E|^{2}+\frac{1}{2} \mu_{0} \omega \operatorname{Im} \mu(\omega)|H|^{2} .
\end{aligned}
$$

From the above equation it can be observed that the rate of energy that dissipates in the absorptive and dispersive medium takes into account both the real part and imaginary parts of the electric permitivitty and magnetic permeability. Hence, the relation can be seen by Equation 2.34 whereby the amount of heat that is absorbed in the system is converted to heat which is governed by the above equation. 


$$
A=\int_{v} q d V
$$

\subsection{Narrow-Band Perfect Metamaterial Absorber (PAs)}

The concept of creating a PA is embedded in the fact that in order to develop a unity absorber, an absorbance, $A(\omega)$, of $100 \%$ is required. Therefore, one needs to reduce "transmittance", $T(\omega)$, as well as reduce "reflectance", $R(\omega)$, to value of zero. This relation is given in the Equation 2.37. The reflectance can be taken to a value of zero by decoupling $\epsilon$ and $\mu$ and then individually tuning their respective frequencies such that $\epsilon=\mu$. Once this has been achieved, the MM composite can be impedance-matched to free space resulting in zero reflectance. A perfectly impedance mathced MM composite can result in zero reflectance but making the transmittance go to zero is a tricky business which is essential in order to realize an absorbance of zero. The transmittance of the MM composite can then be tailored, for example, through the addition of a dielectric layer so that the it goes to zero or through the addition of multiple layers, hence, achieving an unity absorbance. Of importance is to note using this procedure to achieve unity absorbance is through the manipulation of the real parts of the $\epsilon$ and $\mu$. On the other hand, it is also feasible to obtain a high absorbance by boosting one or both the imaginary parts of the $\epsilon$ and $\mu$. Equations (2.37-2.36) represents the formula to calculate absorbance following the Electromagnetic Wave Absorption Theory (see Section 2.5.1) whereby Equation 2.36 shows the same calculation performed using complex frequency dependent 2-port S-parameters, $S_{11}$ and $S_{21}$.

$$
\begin{aligned}
& A(\omega)=1-T(\omega)-R(\omega) \\
& A(\omega)=1-\left|S_{21}\right|^{2}-\left|S_{11}\right|^{2}
\end{aligned}
$$




\subsubsection{Perfect Impedance Matching}

Impedance matching or more accurately impedance matching to free space is a widely used tool employed in the metamaterials research community. It is employed to create perfect or broadband absorbers by bringing the reflection from the metal-dielectric interface to zero. The perfect impedance matching (PIM) $[13,46,16]$ theory states that in order to achieve a condition of zero reflection, the effective impedance has to be equal to the impedance of the free space, that is, $Z=Z_{0}$. The PIMs theory can be used to explain the perfect absorbance characteristic or MMs as shown by the equation below given that the transmittance has been eliminated through the use of a thick ground plane.

$$
\begin{aligned}
A(\omega) & =1-T(\omega)-R(\omega) \\
& =1-R(\omega)
\end{aligned}
$$

Equation 2.38 represents effective impedance and Equation 2.39 represents the impedance of the free space.

$$
\begin{aligned}
Z & =\sqrt{\frac{\mu}{\epsilon}} \\
Z_{0} & =\sqrt{\frac{\mu_{0}}{\epsilon_{0}}}
\end{aligned}
$$

Given that reflection can be represented by Equation [16],

$$
R(\omega)=\left|\frac{Z-Z_{0}}{Z+Z_{0}}\right|^{2}
$$

The perfect absorbance can be evaluated as shown below, 


$$
\begin{aligned}
A(\omega) & =1-R(\omega) \\
& =1-\left|\frac{Z-Z_{0}}{Z+Z_{0}}\right|^{2} \\
& =1-\left|Z-Z_{0}\right|^{2} \\
& =1-\left|Z-Z_{0}\right| \\
& =1-\left|\sqrt{\frac{\mu}{\epsilon}}-\sqrt{\frac{\mu_{0}}{\epsilon_{0}}}\right| .
\end{aligned}
$$

The above expression is of immense importance as it has been fundamentally employed to achieve perfect or broadband absorbance. The parameters in the above expression can be calculated using S-parameters through tailoring one or a combination of them and then perfect impedance matching to free space can be attained via electrical, $\epsilon$, or magnetic, $\mu$ coupling or decoupling.

\subsubsection{Resonant Absorbers}

\subsubsection{Previous Modeling Designs}

\subsubsection{Split Ring Resonators}

One of the most widely used metamaterial device is a electric ring resonator(ERR) as illustrated in the Figure 2.9. Landy et al. [13] fashioned a narrow band perfect absorber using a dual-paired electric ring resonator connected using using a cut wire (CW) as can be observed in Figure 2.17 a). In his research, by changing the geometry of the cut wire and the separation distance of ERR and CW, he was effectively able to tune the magnetic response without changing the ERR and eventually decouple $\epsilon$ and $\mu$ and tune each the resonances individually. He then applied a similar procedure outlined in Section 2.7 to achieve a perfect metamaterial absorber. The simulations verified by experimental results displayed a sharp, narrow peak in the $\mathrm{GHz}$ regime at $11.2 \mathrm{GHz}$. The set back of Landy's PA was that it was 
polarization sensitive which had been improved upon by researchers Wakatsuchi et al. [47] and Tuong et al. [14]

\subsubsection{Parallel Cut Wires}

In a similar study to the one conducted by Landy, Tuong et al. [14] conducted an investigation on the relationship between the absorbance and the reflectance of an I-shaped MM composite, designed as a repeating pattern of metal (ERR)-dielectric-metal (CW) scheme. The dielectric component, FR-4, was sandwiched between two lossy copper components. It was a modified structure from the Landy's dual-shaped ERR with unique difference in design being that the $\mathrm{C}$ corners of the dual-electric ring resonators were removed to create the $\mathrm{I}$ structure as represented in the Figure 2.17.
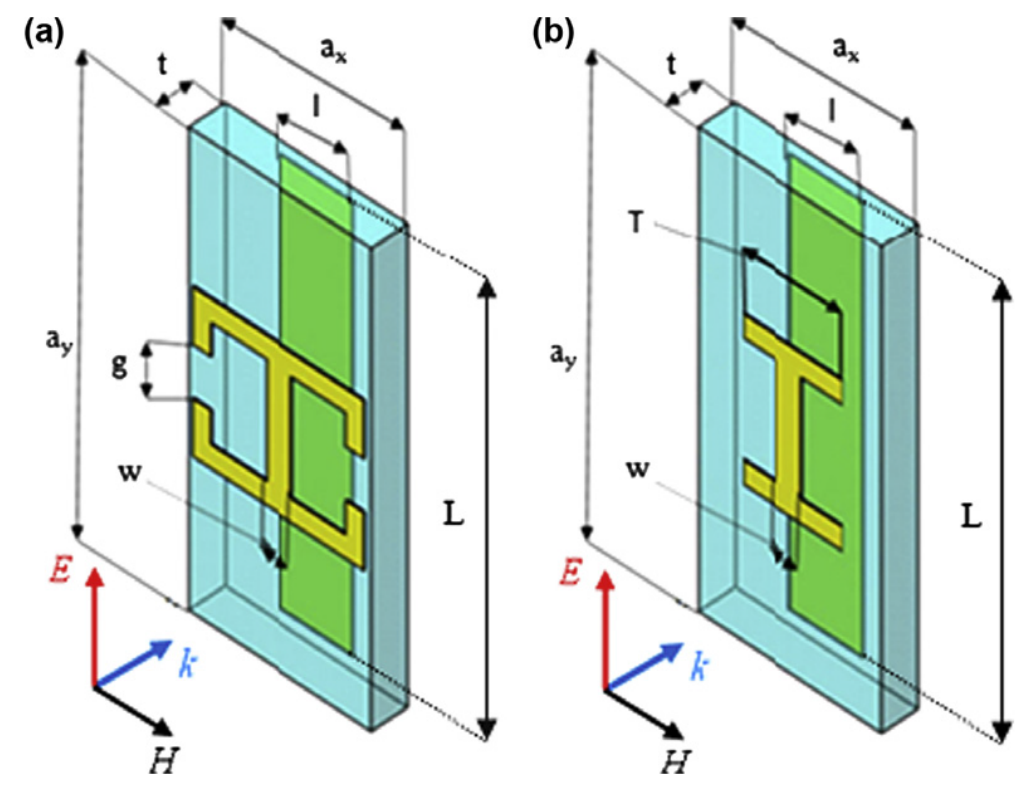

Figure 2.17 Schematic representation of Landy et al. [13] origianl structure (a) and the Tuong et al. [14] modified structure (b).

Toung discovered a strong correlation between reflection, due to the "g" in the "C" 
corners and absorbance since transmittance was almost totally extinct in the dielectric medium. she was able to demonstrate a much simpler structure as compared to Landy's which was still able to achieve a sharp, resonant peak in the GHz region just by changing the geometry of ERR without changing the separation distance or the center. The simplified I-shaped structure is yet another testimony of the flexibility and scalability of a MM composite and its strong dependence and on the configuration of its building blocks.

\subsubsection{Even Leaf Shapes in Nature}

The spectral resonant peaks of perfect absorbers as shown by many researchers [39, 48, 49] has been unsymmetrical. In order to improve upon the symmetry of PAs, researchers $[50,51,52]$ have focused considerable attention in the symmetrical nature of their design which becomes yet another salient feature of PAs. This is because in addition to the near unity peaks, it also caters for polarization insensitivity of the incoming illuminance. In such a research, Tuong et al. [27] was able to demonstrate a perfectly polarization-independent structure through the utilization of a four petaled flower-shaped structure (FSS). The FSS, constructed of an intersection of two overlapping circles, boasted an extremely easy to customise frequency peak by varying the radius of the circles, thus, adding an additional advantage to it.

Employing a similar three layer design of metal-dielectric-metal sandwich model as mentioned above [14, 27], she was able to demonstrate an even sharper and more symmetrical peak as compared to earlier works $[13,14,47]$. Such a sharp, well defined, perfect absorber would be a huge contribution in applications like bolometers.

\subsection{Broadband Metamaterial Absorber}

\subsubsection{Multiple sub-cells within the unit cell}

In all the above cases, of great interest has been to achieve a sharp resonant peak almost close to unity arising from the plasmon resonance of the controlled structured nano- 
particle arrangement of the electromagnetic metamaterial and controlling the position of the peak. However, the concept of broadband or multi-band has begun to surface when the shape of these resonance peaks were given greater emphasis [53]. This has led to increase efforts in the creation of broadband perfect absorbers. Berkovitch et al. [53] has been able to demonstrate a broadband absorber in the Near Infrared Regime (NIR) which employs two resonant plasmonic peaks conceived by constructing a unit cell with two nanoantennas (transverse and longitudinal arrangement) of typical sizes greater than 50nm while at the same time trying to reduce coupling between them. He was able to achieve this by demonstrating that plasmonic behavior of nanoparticles is "wide" which is a result of Ohmic losses by the imaginary part of the materials permiitivity and scattering of light by the particles via radiation losses. He used a energy splitting scheme for the different nanoantennas to suppress coupling. In a similar research, which is also an extension of Landy's cut-wires, Wakatsuchi et al. [47] was able to characterize a broadband absorber by careful, periodic arrangement of several cut-wires within the same unit cell. He deduced from his investigations that the CW metamaterials had two types of resonances: electric and magnetic and by tailoring them individually and then combining them a broadband spectrum in the $\mathrm{GHz}$ regime can be attained. The idea of introducing multiple CWs was to obtain many absorption peaks which through interaction with other polarization can result in a broadband. Furthermore, he showed the customisable absorptance characteristics of each of this CWs were dependent on the "lossy and the resistive" nature of the CWs when placed on perfect electric conductors (PEC). Wakatsuchi's research was further verified numerically by Wang et al. [25] who used multiple SRR as part of his unit celled metamaterial to show the correlation between the absorption peaks and the electric and magnetic resonances.

\subsubsection{Fractal plasmonic metamaterial broadband aborber}

In addition to the above mentioned practice of achieving a broadband spectrum through multiple sub-cells combined within a unit cell, fractal designs also provide a very 
useful platform to design and characterize perfect broadband absorbers. This is because fractals provide self-similar, space filling and sub-wavelength perforations on the manufactured MM surface which can represent every single wavelength of the visible regime. Maragechi et al. [48] utilized the widely studied Sierpinski's gasket fractal design to show the increase in absorptance power and enhanced $\mathrm{THz}$ radiation emission with increasing orders of fractal design. He attributed this increase to the increased broadband at various frequencies as a result of multi-resonances achieved by the fractal configuration which in turn induced coupling between the sub-wavelength structures. 


\section{CHAPTER 3}

\section{FINITE-DIFFERENCE TIME-DOMAIN (FDTD) SIMULATION}

In order to understand the plasmonic resonance effects and its relation with the electromagnetic radiation propagating through the macroscopic metamaterial composite, it is essential to study Maxwell's Equations separately for the noble metals and the dielectric media as within their framework the behavior of EM waves in metals and dielectrics can be completely characterized. The polarization of matter as discussed in Section 1.1

\subsection{Maxwell's Equations}

Maxwell's equations not only satisfy electromagnetic radiation of the short wavelength but also of longer wavelengths as demonstrated by Hertz in any medium. The general macroscopic Maxwell's equations which also accounts for the properties of the medium [2, $1,54,55]$, can be written in the differential form as follows;

$$
\begin{gathered}
\nabla \times \vec{E}=-\frac{\partial \vec{B}}{\partial t}, \\
\nabla \times \vec{H}=\vec{J}+\frac{\partial \vec{D}}{\partial t}, \\
\nabla \bullet \vec{D}=\rho_{c}, \\
\nabla \bullet \vec{B}=0 .
\end{gathered}
$$


Where, $\vec{E}$ is the electric field in $\frac{V}{m}, \vec{H}$ is the magnetic field in $\frac{A}{m}, \vec{J}$ is the electric current density in $\frac{A}{m^{2}}, \vec{D}$ is the electric displacement in $\frac{C}{m^{2}}, \vec{B}$ is the magnetic flux density in $\frac{W b}{m^{2}}$ and $\rho$ is the charge density in $\frac{C}{m^{3}}$.

\subsubsection{Maxwell's Equations in Noble Metals}

Additional considerations to relate $\vec{D}$ and $\vec{H}$ to other fields are implemented to give $\vec{D}=\epsilon_{0} \vec{E}$ and $\vec{B}=\mu_{0} \vec{H}$ and since silver is a conducting medium, Ohm's law for a conducting medium is taken into account, $\vec{J}=\sigma \vec{E}$. Whereby, $\epsilon_{0}$ is the permittivity, $\mu_{0}$ is the permeability and $\sigma$ is the electrical conductivity. Hence, the Maxwell's equations for the noble metals can be written as follows;

$$
\begin{gathered}
\nabla \times \vec{E}=-\frac{\partial \vec{B}}{\partial t}=-\mu_{0} \frac{\partial \vec{H}}{\partial t}, \\
\nabla \times \vec{H}=\vec{J}+\frac{\partial \vec{D}}{\partial t}=\sigma \vec{E}+\epsilon_{0} \frac{\partial \vec{E}}{\partial t}, \\
\nabla \bullet \vec{D}=\rho_{c}, \\
\nabla \bullet \vec{B}=0 .
\end{gathered}
$$

Taking the curl of Equation 3.5 and combing it with Equation 3.6 yields the charge conservation or continuity equation as shown below.

$$
\nabla \times \nabla \times \vec{E}+\mu_{0} \sigma \frac{\partial \vec{E}}{\partial t}+\mu_{0} \epsilon_{0} \frac{\partial^{2} \vec{E}}{\partial t^{2}}=0
$$


By applying the vector identity

$$
\nabla \times \nabla \times(\ldots)=\nabla(\nabla \bullet \ldots)-\nabla^{2}(\ldots)
$$

to Equation 3.9 and assuming no charges are present, the following wave equation for $\vec{E}$ is obtained.

$$
\nabla^{2} \vec{E}-\mu_{0} \sigma \frac{\partial \vec{E}}{\partial t}-\mu_{0} \epsilon_{0} \frac{\partial^{2} \vec{E}}{\partial t^{2}}=0
$$

By taking the curl of Equation 3.6 and combining it with Equation 3.5, the corresponding wave equation for $\vec{H}$ is obtained.

$$
\nabla^{2} \vec{H}-\mu_{0} \sigma \frac{\partial \vec{H}}{\partial t}-\mu_{0} \epsilon_{0} \frac{\partial^{2} \vec{H}}{\partial t^{2}}=0
$$

\subsubsection{Maxwell's Equations in Dielectrics}

Similarly, Maxwell's equations for waves in the dielectric media can be written with the exception that there are no additional free charges than those bound at the atomic level. To write these equations for the dielectric media, additional considerations such as those in Section 3.1.1 are taken into account to relate $\vec{D}$ and $\vec{H}$ to other fields. These relations are $\vec{D}=\epsilon_{0} \vec{E}+\vec{P}$ and $\vec{B}=\mu_{0} \vec{H}+\vec{M}$ whereby $\vec{P}=\chi \epsilon_{0} \vec{E}$ and $\vec{M}=\chi \mu_{0} \vec{H}$ where $\vec{P}$ is the polarization vector and $\chi$ is the displacement. Hence, the Maxwell's equations for the dielectric media can be written as follows:

$$
\begin{gathered}
\nabla \times \vec{E}=-\frac{\partial \vec{B}}{\partial t}, \\
c^{2} \nabla \times \vec{B}=\frac{\partial}{\partial t}\left(\frac{\vec{P}}{\epsilon_{0}}+\vec{E}\right), \\
\nabla \bullet \vec{E}=-\frac{\nabla \bullet \vec{P}}{\epsilon_{0}},
\end{gathered}
$$




$$
\nabla \bullet \vec{B}=0
$$

Following similar procedures as in Equation 3.9 - 3.10, the wave equation in terms of polarization for the dielectric media can be written as shown below;

$$
\nabla^{2} \vec{E}-\frac{1}{c^{2}} \frac{\partial^{2} \vec{E}}{\partial t^{2}}=-\frac{1}{\epsilon_{0}} \nabla(\nabla \bullet \vec{P})+\frac{1}{\epsilon_{0} c^{2}} \frac{\partial^{2} \vec{P}}{\partial t^{2}}
$$

\subsection{Numerical Solutions for Maxwell's equations}

In order to facilitate the easy calculation and simulation of the above mentioned equations, we employ the commercial Computer Simulation Technology (CST) package with various inbuilt solvers. CST Microwave Studio is a 3D high frequency domain solver which was used to construct the model and predict the scattering response. The method that has been used extensively in my research is the Finite-Difference-Time-Domain(FDTD) through the application of the Frequency Domain Solver as well as the transient solver.

\subsection{Model Progression}

The following paragraphs guide us through the creation and simulation of first and higher order absorbers and the process that is adapted in the optimization and tunability of these absorbers.

\subsubsection{Constructing the CAD model}

The absorbers, as presented in Figure 3.1, are created using a bottom up approach. A "metal-dielectric-metal" or "sandwich" model is generally implemented by first specifying a thick metallic ground plane to eliminate any transmission that might occur during the simulation process, followed by the dielectric part, the metallic nano-particle antenna and a 
layer of vacuum (not shown here). More specifically all of our models are constructed using "silver- $\mathrm{Al}_{2} \mathrm{O}_{3}$-silver" sandwich scheme. The purpose of simulating our models in vacuum is to eliminate any scattering or dispersion that would occur in the presence of particulates in the air. This is to ensure that the total normal incident radiation is reaching the top surface of the model in question.
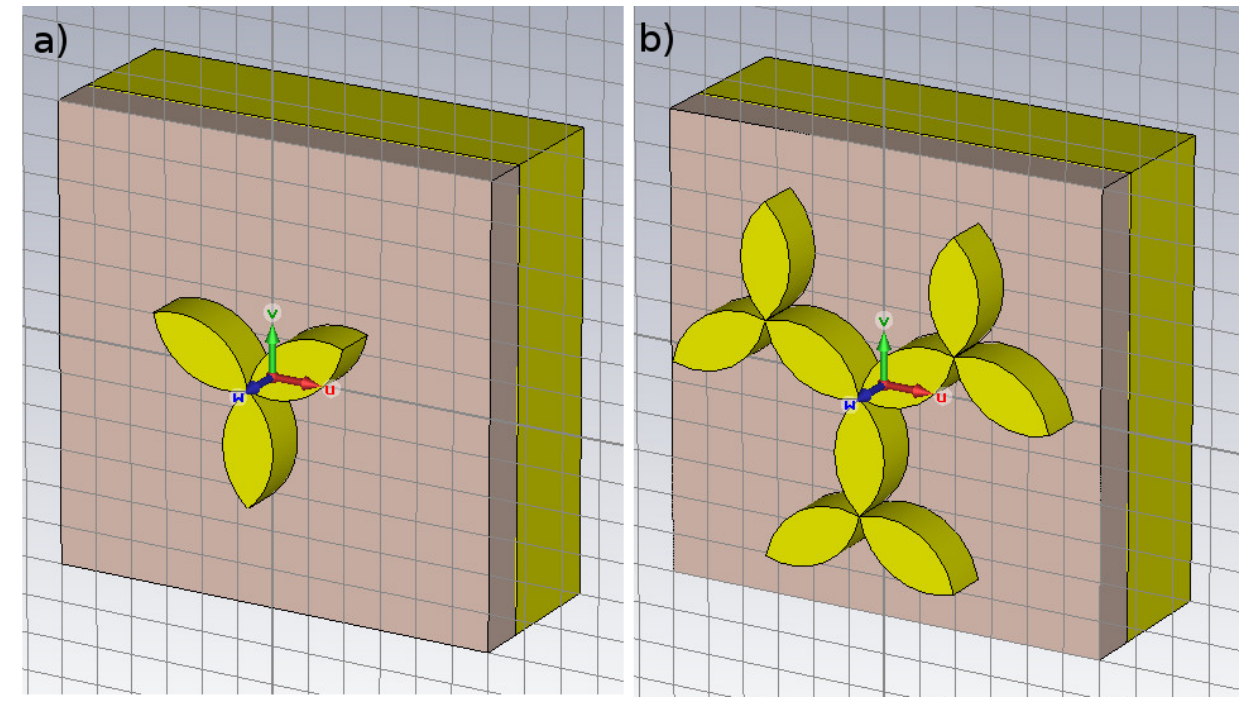

Figure 3.1 a) Schematics of a first order petal shaped fractal nano-antenna. b) Second order petal shaped fractal nano-shaped antenna.

\subsubsection{Determine the Material Properties}

The material properties of the silver, a lossy material, had an electrical conductivity of $6.3012 \times 10^{7} \mathrm{~S} / \mathrm{m}$ and $\mu_{0}$ of 1 . The silver was characterized by the drude model as discussed in Section 2.5.3.1 with plasma frequency of $\omega_{p}=1.37 \times 10^{16} \mathrm{~s}^{-1}$ and the collision frequency $\gamma=8.5 \times 10^{-12}$. For the visible regime, this model was deemed sufficient to describe the motion of free electrons inside the the silver metal.

The alumina $\left(\mathrm{Al}_{2} \mathrm{O}_{3}\right)$, a $96 \%$ lossy material, was characterized by a pole-zero disper- 


\begin{tabular}{|c|c|c|c|}
\hline \hline Constituent & Parameter & Description & Parameter Value $(\mathrm{nm})$ \\
\hline \hline \multirow{3}{*}{ Nanoparticle } & $W_{p}$ & particle width & 50 \\
& $L_{p}$ & particle length & 50 \\
& $T_{p}$ & particle thickness & 30 \\
\hline \multirow{3}{*}{ Dielectric } & $W_{d}$ & dielectric width & 60 \\
& $L_{d}$ & dielectric length & 60 \\
& $T_{d}$ & dielectric thickness & 12 \\
\hline \multirow{3}{*}{ Substrate } & $W_{s}$ & substrate width & 60 \\
& $L_{s}$ & substrate length & 60 \\
& $T_{s}$ & substrate thickness & $\infty$ \\
\hline
\end{tabular}

Table 3.1 Critical parameters used in the construction of a nano-particle sized unit celled antenna for the First Order Absorber design.

sion model with a electric tangent delta of 0.0004 at $1 \mathrm{MHz}$. This tangent dispersion model for the dissipation of electromagnetic energy is equivalent to the first order Debye model. In comparison to a Debye model, the real part of the permittivity, $\epsilon_{0}^{\prime}$, is 9.4 and the imaginary part of the permittivity, $\epsilon_{0}^{\prime \prime}$ varies from $1.0 \times 10^{-12}$ to $2.6 \times 10^{-12}$ with a $\mu_{0}$ of 1 . The differences in these permittivity values, contribute varying electric and magnetic response to time varying electromagnetic radiation at a certain resonance frequency, thus, providing a frequency dependent response of the dielectric material. All materials in the construction of the nano-antenna is considered non-magnetic, that is, $\mu=\mu_{0}$ and homogenous. Table 3.1 describes the commonly used parameters in the construction of a unit-celled nano-antenna.

\subsubsection{Periodic Boundary Conditions}

In order to imitate the macroscopic structure of this simple nano-antenna design, our simple unit-celled cuboidal antenna is simulated using periodic boundary conditions which extends infinitely in the $\hat{x} \hat{y}$-plane as depicted in the Figure 3.2. This makes our simulation time and effort tremendously easier as only the single unit cell has to be studied which can be extrapolated for the macroscopic structure. 


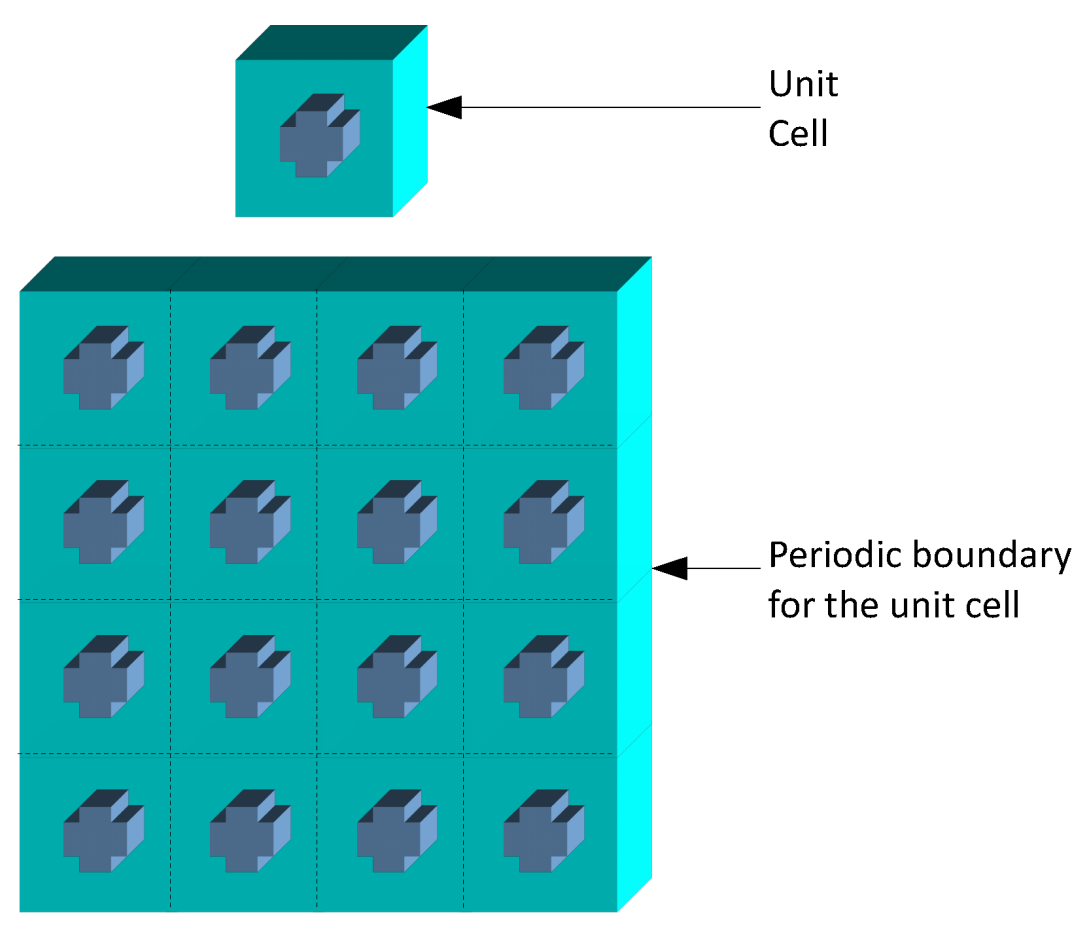

Figure 3.2 Illustration of the periodic boundary condition for the unit-celled structure).

\subsubsection{Plane Wave Excitation and Wave Ports}

A transverse electric (TE) plane wave is specified at the top of domain and propagated in the negative z-direction. Two ports were created in the positive and negative $z$ - direction as illustrated in Figure 3.3. The plane polarized TEM light was simulated in the $-z$ to $+z$ direction, that is, perfectly electric in the $\hat{y} \hat{z}$-plane and perfectly magnetic in the $\hat{x} \hat{z}$-plane. The response of the structure is then monitored for a range of electromagnetic radiation from a frequency of $300 \mathrm{THz}$ to $800 \mathrm{THz}$.

\subsubsection{FDTD simulation}

\subsubsection{Time Domain Solver}

Solves the aforementioned Maxwell's equations for metal and dielectric in time. The purpose of the time domain solver is to retrieve S-parameters for various S-parameter or 


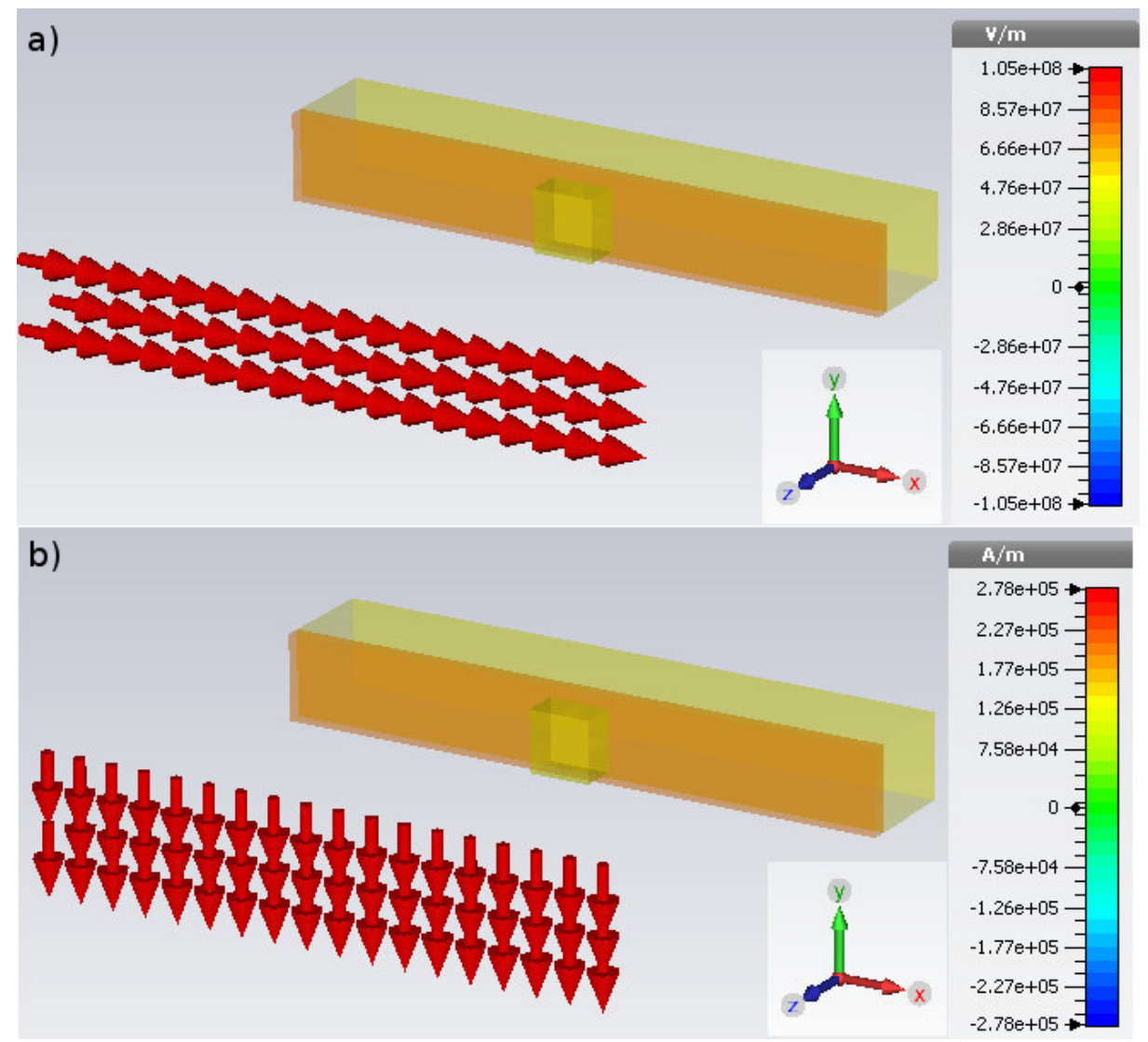

Figure 3.3 The electric and the magnetic components of the waveguide at the inlet port.

antenna problem. By simulating the structure at a prior port definition via a broadband signal, it enables the s-parameters to be obtained for the entire desired frequency regime.

\subsubsection{Frequency Domain Solver}

Solves the aforementioned Maxwell's equations for metal and dielectric in frequency space. Similar to the transient solver, the frequency domain solver obtains the S-parameters. The advantage of the frequency domain solver over the time domain solver lies in the fact that it can easily and quickly calculate S-parameters when the response of a carefully chosen limited number of samples in a preferable frequency domain needs to be performed. This proves more efficient in terms of simulation time and runs especially for a fewer number of mesh cells. 


\subsubsection{Field Monitors}

Field monitors are applied to the simulations to more accurately visualize and provide a greater insight at the behavior of electromagnetic distribution inside the plasmonic metamaterial structures. It can be defined using the frequency or the time. In my research the field monitors were placed at the resonant frequencies of the surface plasmons to study the behavior of the structures. The field monitors that were employed in my cases were the following: 1) The electric field monitor describes the distribution of the electric field at the resonant frequencies. 2) The magnetic field monitor describes the magnetic field distribution at the resonant frequencies. 3) The Surface Current Density Monitor uses the mgnetic fields on the surfaces of lossy materials to calculate the surface currents there. 4) The power flow monitor stores the pyonting vector of the electromagnetic field and 5) The power loss density monitor calculates the specific absorption rates at the specified frequency usually divided over a specified mass.

\subsubsection{Scattering Parameters or S-parameters}

S-parameters has been one of the most versatile tool that has been extensively employed to interpret more clearly our numerical results. All of our modeling designs were simulated using a two-port network system as shown in the Figure 3.4. The scattering parameters are calculated from the normalized intensity at the both of the ports to assist in our understanding of the absorption at the resonant frequencies.

$$
\begin{aligned}
& a_{1}=\frac{E M_{1}+I_{1} \sqrt{Z_{0}}}{2 \sqrt{Z_{0}}} \\
& a_{2}=\frac{E M_{2}+I_{2} \sqrt{Z_{0}}}{2 \sqrt{Z_{0}}}
\end{aligned}
$$




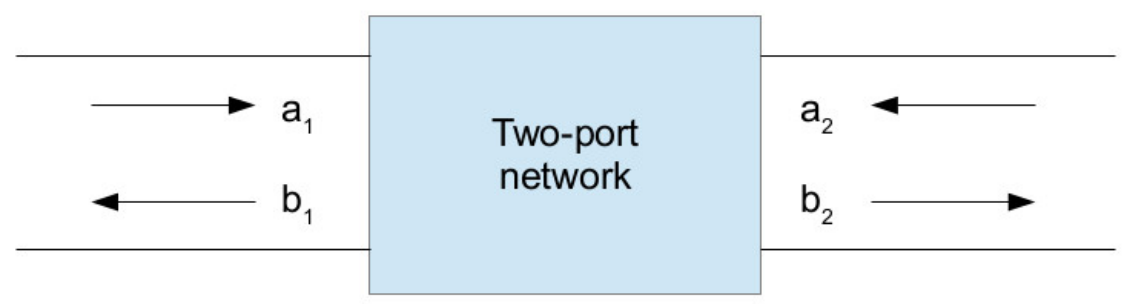

Figure 3.4 Schematics of normalized s-parameters applied in the calculation of absorptance.

$$
\begin{aligned}
& b_{1}=\frac{E M_{1}-I_{1} \sqrt{Z_{0}}}{2 \sqrt{Z_{0}}} \\
& b_{2}=\frac{E M_{2}-I_{2} \sqrt{Z_{0}}}{2 \sqrt{Z_{0}}}
\end{aligned}
$$

Where $a_{1}$ and $b_{1}$ are incident electromagnetic radiation and $Z_{0}$ is the impedance. The relations between the s-parameters and $a_{1}$ and $b_{1}$ are expressed in the Equations 3.22 - 3.24.

$$
\begin{aligned}
& s_{11}=\left|\frac{b_{1}}{a_{1}}\right|_{a_{2}=0}=\frac{E M_{1}-I_{1} \sqrt{Z_{0}}}{E M_{1}+I_{1} \sqrt{Z_{0}}} \\
& s_{21}=\left|\frac{b_{2}}{a_{1}}\right|_{a_{2}=0}=\frac{E M_{2}-I_{2} \sqrt{Z_{0}}}{E M_{1}+I_{1} \sqrt{Z_{0}}} \\
& s_{22}=\left|\frac{b_{2}}{a_{2}}\right|_{a_{2}=0}=\frac{E M_{2}-I_{2} \sqrt{Z_{0}}}{E M_{2}+I_{2} \sqrt{Z_{0}}} \\
& s_{12}=\left|\frac{b_{1}}{a_{2}}\right|_{a_{2}=0}=\frac{E M_{1}-I_{1} \sqrt{Z_{0}}}{E M_{2}+I_{2} \sqrt{Z_{0}}}
\end{aligned}
$$




\subsubsection{Absorption Calculation}

These scattering parameters were then used to define the absorptivity and the reflectivity from the view point of the top most port above the device. Because the silver substrate had a sufficient thickness, the transmissivity from port 1 to port $2\left(\mathrm{~S}_{12}\right)$ was assumed and confirmed by the simulation to be zero. This resulted in simplification of the scattering parameters. Defining a energy balance, $\mathrm{A}(\omega)+\mathrm{R}(\omega)+\mathrm{T}(\omega)=1$, where $\mathrm{A}(\omega)$ is the absorptivity, $R(\omega)$ is the reflectivity and $T(\omega)$ is the transmissivity. The reflectivity can be defined as scattering parameter $R(\omega)=\left|S_{11}\right|^{2}$ and hence the absorptivity can be defined as $\mathrm{A}(\omega)=1-\left|S_{11}\right|^{2}$. Equations 3.26 - 3.27 describes the relationship between the sparameters, absorption and ultimately the heat generation in the plasmonic meta-material nano structures.

$$
\begin{aligned}
& A(\omega)=1-T(\omega)-R(\omega) \\
& A(\omega)=1-\left|S_{21}\right|^{2}-\left|S_{11}\right|^{2}
\end{aligned}
$$

The equations below show the relation between the s-parameters and the impedance matching which if of great interest since impedance matching has been extensively employed to obtain zero reflection.

$$
Z= \pm \sqrt{\frac{\left(1+S_{11}\right)^{2}-S_{21}^{2}}{\left(1-S_{11}\right)^{2}-S_{21}^{2}}}
$$

Equation 3.28 can be reduced to Equation 3.29 because of $S_{21}$ tending to zero as a result of thick ground plane.

$$
Z= \pm \sqrt{\frac{\left(1+\left|S_{11}\right|\right)^{2}}{\left(1-\left|S_{11}\right|\right)^{2}}}= \pm \frac{\left(1+\left|S_{11}\right|\right)}{\left(1-\left|S_{11}\right|\right)}
$$




$$
\begin{gathered}
Z=\sqrt{\frac{\mu}{\epsilon}}=\sqrt{\frac{\mu_{1}+i \mu_{2}}{\epsilon_{1}+i \epsilon_{2}}} \\
Z=\sqrt{\frac{\mu}{\epsilon}}=\sqrt{\frac{\mu_{1}+i \mu_{2}}{\epsilon_{1}+i \epsilon_{2}}}=\sqrt{\frac{\left(1+\left|S_{11}\right|\right)^{2}}{\left(1-\left|S_{11}\right|\right)^{2}}} \\
\frac{\mu}{\epsilon}=\frac{\mu_{1}+i \mu_{2}}{\epsilon_{1}+i \epsilon_{2}}=\frac{\left(1+\left|S_{11}\right|\right)^{2}}{\left(1-\left|S_{11}\right|\right)^{2}} \\
\left|S_{11}\right|=\sqrt{S_{11_{R}}^{2}+S_{11_{I}}^{2}} \\
\frac{\mu}{\epsilon}=\frac{\mu_{1}+i \mu_{2}}{\epsilon_{1}+i \epsilon_{2}}=\frac{\left(1+\left(\sqrt{S_{11_{R}}^{2}+S_{11_{I}}^{2}}\right)\right)^{2}}{\left(1-\left(\sqrt{S_{11_{R}}^{2}+S_{11_{I}}^{2}}\right)\right)^{2}}
\end{gathered}
$$

\subsubsection{Model Modification}

Once the simulation has been conducted and the s-parameters extracted, the field monitors are studied at the desired resonant frequencies to analyze the behavior of the EM radiation at the resonant modes. The parameters exhibiting desirable properties are noted which are used for the creation of higher order absorbers or to allow for the tunability of the spectral peak by individually tuning the magnetic and/or electric resonance or the real or imaginary part of the electric permitivitty or magnetic permeability. Depending on the nature of the need, the steps from subsections 3.3.5 - 3.3.8 are recalculated and similar analysis conducted. 


\section{CHAPTER 4}

\section{COMPUTATIONAL RESULTS}

\subsection{Design Rationale}

Since the entire objective of our studies is to create a perfect broadband absorber in the visible region, this research commenced with a thorough study of perfect peak absorbers (PAs) in the various regimes of the electromagnetic spectrum. The examples below show the characterization of perfect absorbers in the $\mathrm{GHz}$ and $\mathrm{THz}$ region and how they can be expanded to create a broadband absorber.

\subsubsection{Perfect Peak Absorbers}

In order to facilitate our understanding of the behavior of PAs, one of the classic prescription, that is, a split-ring resonator was utilized as shown in Figure 4.1 which is a close adaptation of Landy's double split-ring resonator (SRR) [13]. The plane polarized TEM light was simulated in the $-u$ to $+u$ direction, that is, perfectly electric in the $\hat{v} \hat{w}$-plane and perfectly magnetic in the $\hat{u} \hat{w}$-plane. An absorption peak in access of $95 \%$ was recorded in the $\mathrm{GHz}$ regime at $\omega_{0}=11.2 \mathrm{GHz}$ as illustrated in Figure 4.2. In this design, the perfect absorption resulted from impedance matching to free space by matching $\epsilon$ and $\mu$. The results for the split-ring resonator was also verified as presented in Figure 4.3 to show that the correct modeling including appropriate materials properties were utilized. As depicted in Figure 4.1, electric coupling was achieved by the two electric ring resonators and magnetic coupling was achieved by a cut wire (not shown) in the perpendicular direction to the $\hat{u}$. The purpose of the cut wire was to induce anti-parallel current which gives rise to the magnetic response. Therefore, electric response and magnetic response was tailored individually which is essential to decouple $\epsilon$ and $\mu$. This example served to show that perfect absorbers can 
be constructed by employing impedance matching to free space and inducing anti-parallel currents to tune magnetic resonance.

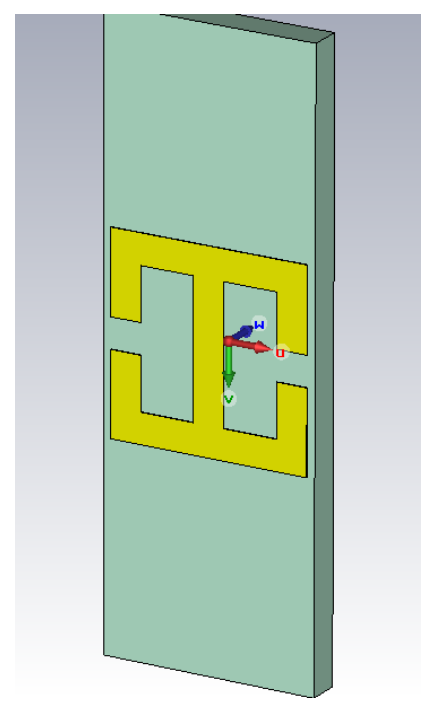

Figure 4.1 Perfect Absorber using a double split-ring resonator and a parallel cut wire at the back to provide individual tuning of electric and magnetic responses.

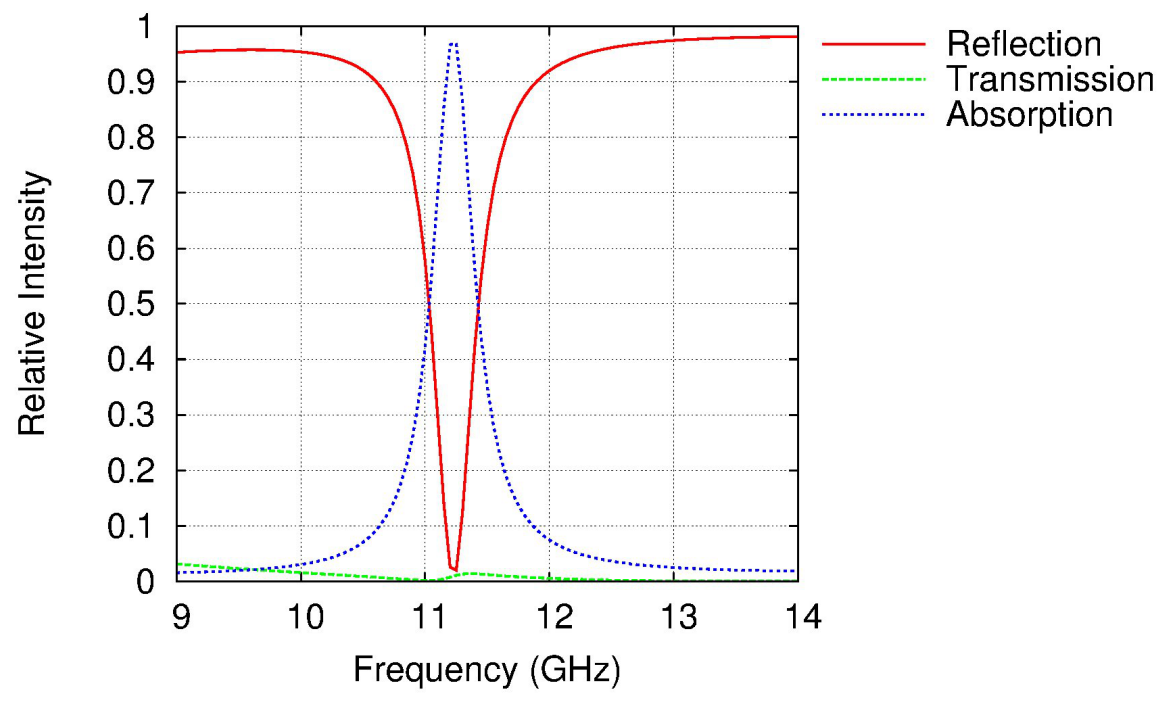

Figure 4.2 Near unity absorption peak in the GHz region $\omega_{0}=11.2 \mathrm{GHz}$. 


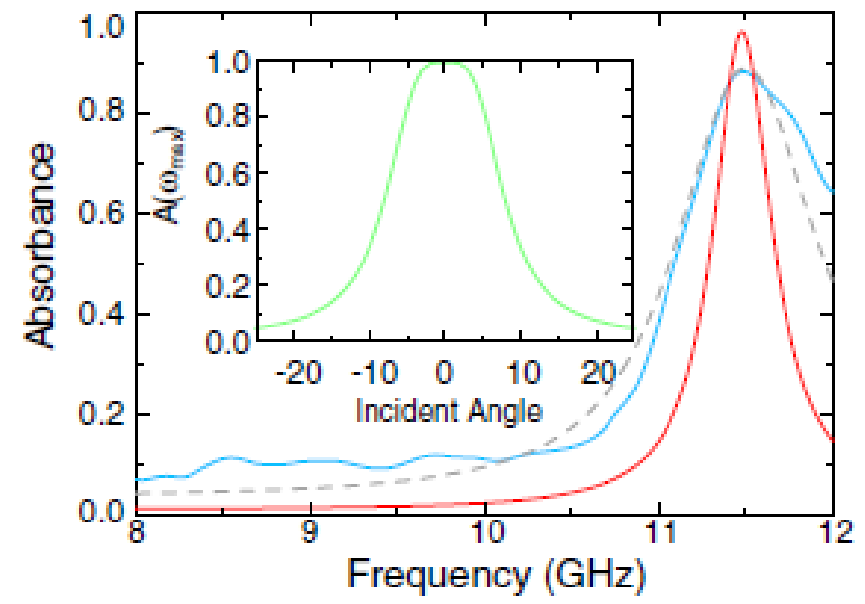

Figure 4.3 Validation of the simulated results of the perfect split ring resonator as shown in Figure 4.2 with experimentation data. The dotted gray lines represents the experimental results as obtained by researcher Landy et. al [13].

\subsubsection{Perfect absorber and its progress to broadband absorber}

Having identified the recipe for creating perfect absorbers, the construction of perfect broadband absorbers is proposed through the utilization of meta-materials composed of fractal design as shown in Figure 4.5. Figure 4.4 shows an absorption peak which was the product of the first order fractal design. In this design the absorption peak was obtained through through the combined effects of the multi-resonance as a result of the fractal geometry.

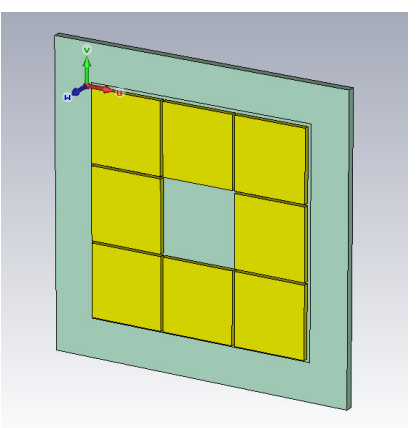

Figure 4.4 First order perfect absorber through the application of fractal design. 


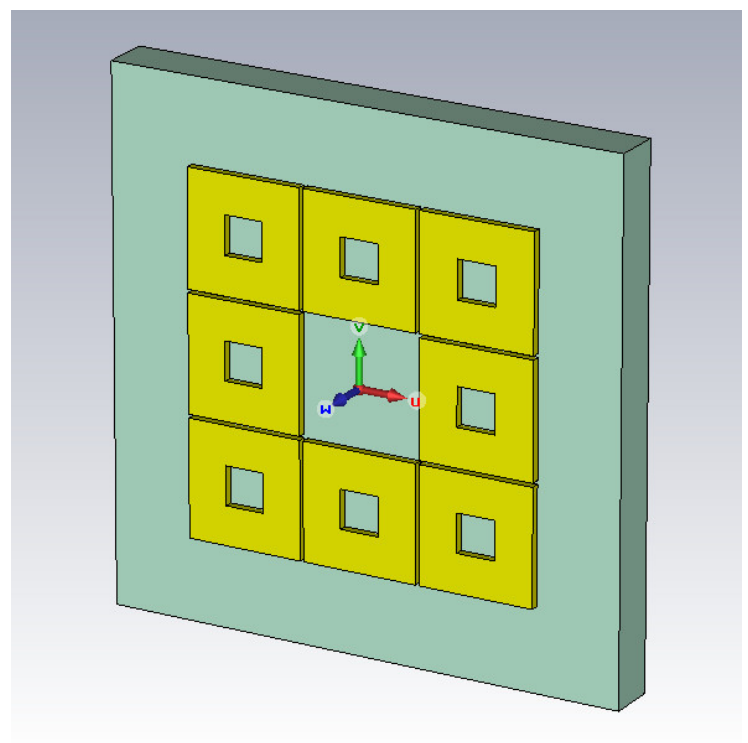

Figure 4.5 Broadband absorber as a result of increasing degree of freedom as a result of engineered fractal design.
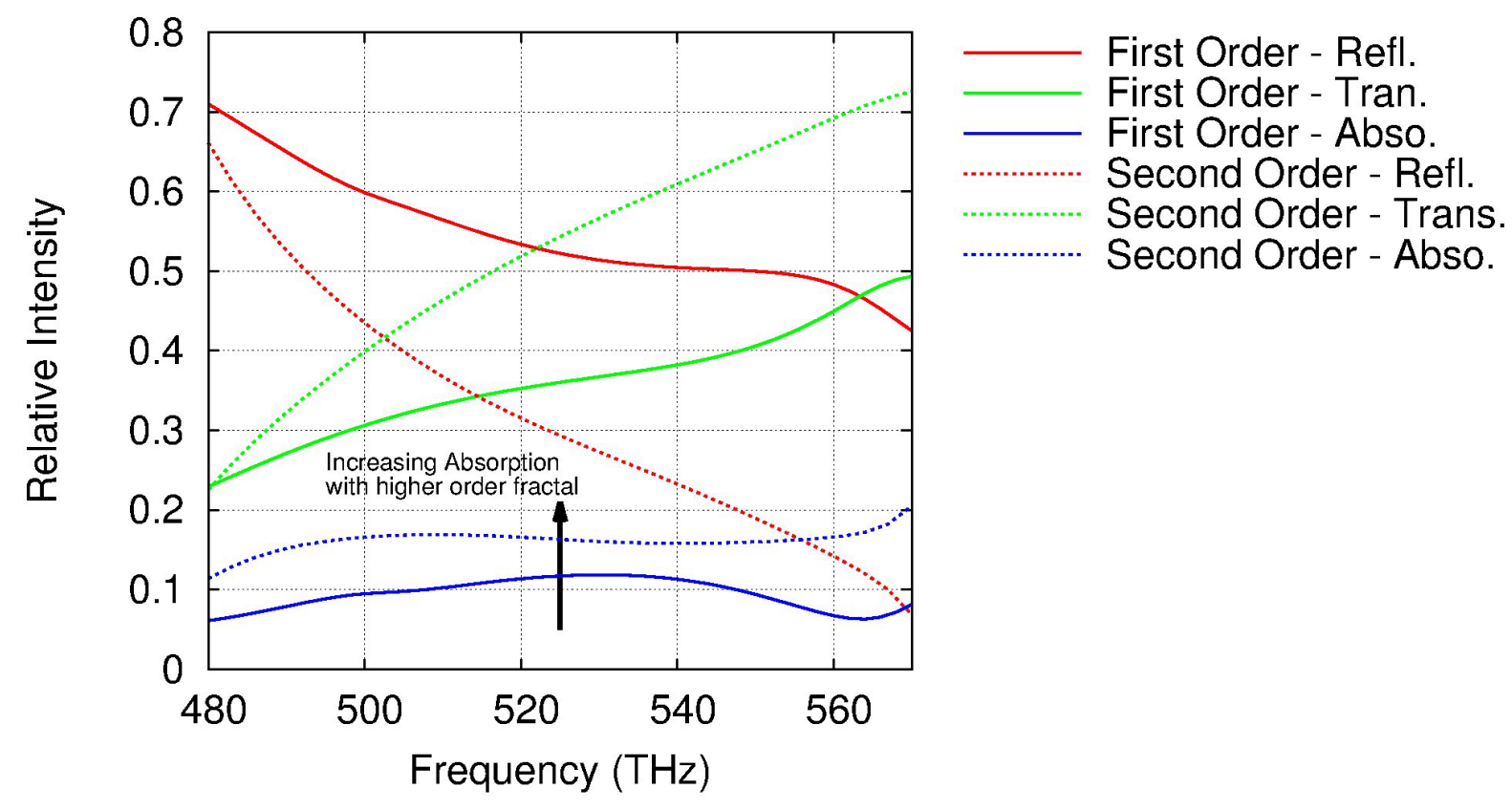

Figure 4.6 A comparison of the bandwidth of the absorption peak in the first order absorber with second order absorber with increasing degree of freedom. 


\subsubsection{Current Fractal Design}

Using the concepts explained in the Sections 4.1.1 and 4.1.2, we strive to characterize a broadband perfect absorber using a simple unit-cell cuboidal shaped nano-antenna as depicted in the Figure 4.7. The numerous peaks that are depicted in Figure 4.8 are a result of multi-resonances from individual squares that we strive to combine to broaden the absorption spectrum and hence improved heat generation over the entire visible regime.
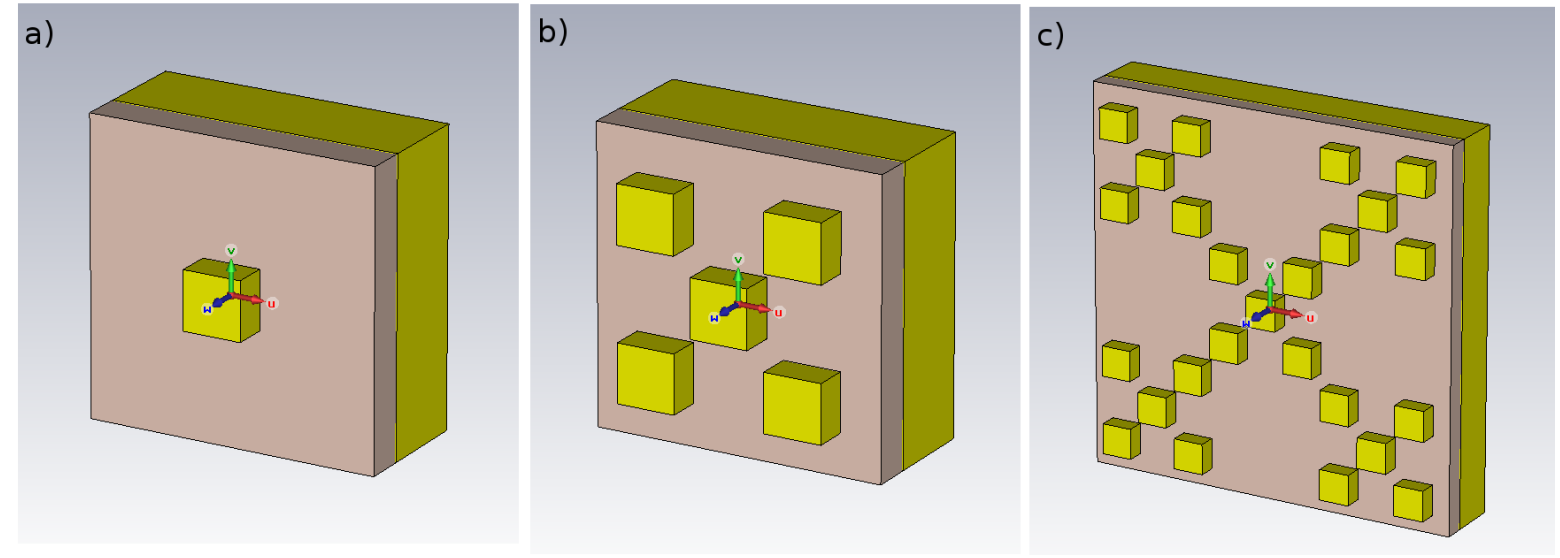

Figure 4.7 a)First order, b) Second order and c) Third Order with increasing order of fractal nano-antennas respectively to create a broadband absorber using the hybridization effect of multi-resonances. 


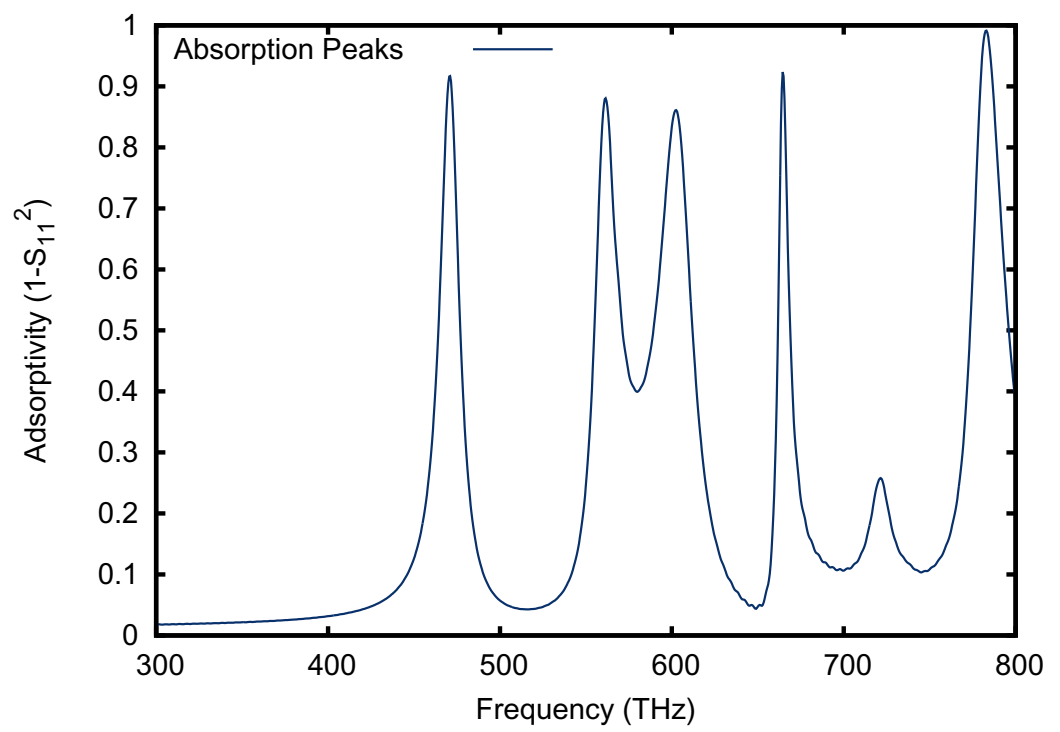

Figure 4.8 Absorptivity response of the second order absorber (see Figure $4.7 \mathrm{~b}$ ), to illustrate the increase in the number of absorption peaks contributing to a broadband absorber.

\subsection{Preliminary Unit Cell Plasmonic Meta-Material Device Design}

\subsubsection{First Order Absorber}

This research commenced with the construction of a unit cell plasmonic device. The geometry of the plasmonic device is illustrated in Figure 4.9. The design is a first order plasmonic structure which utilizes three layers, two metallic layers (silver) and the dielectric layer (alumina) as is depicted in Figure 4.9. A silver layer with dimensions $W_{G} \times L_{G} \times T_{G}$ is used as the substrate (thick ground plane) upon which alumina with dimensions $W_{G} \times$ $W_{G} \times T_{D}$ is deposited. The silver nano-antenna with dimensions $W_{p} \times L_{p} \times T_{p}$ is located in the geometric center on top of the alumina layer. The purpose of this unit cell was to determine the optimal dimensions for the fractal length and the dielectric thickness which would later be used to create higher order fractal plasmonic devices. The plane polarized TEM light was simulated in the $-z$ to $+z$ direction. The incident electromagnetic radiation was perfectly electric in the $\hat{y} \hat{z}$-plane and perfectly magnetic in the $\hat{x} \hat{z}$-plane with periodic boundary condition in the $\hat{x} \hat{y}$-plane as presented in Figure 4.10. Table 4.1 provides the 
description of all the parameters along with the actual values used in the construction of the nano-antenna (Design A). The silver material was assumed lossy and the dispersion of the material was described through a Drude model, see parameters in Table 4.2.1. The dielectric was modeled as alumina $\left(\mathrm{Al}_{2} \mathrm{O}_{3}\right)$ with a lossy Drude model description also found in Table 1. The ground plane had the same material properties as the silver nanoparticle. The air was assumed loss-less with a permitivity of unity

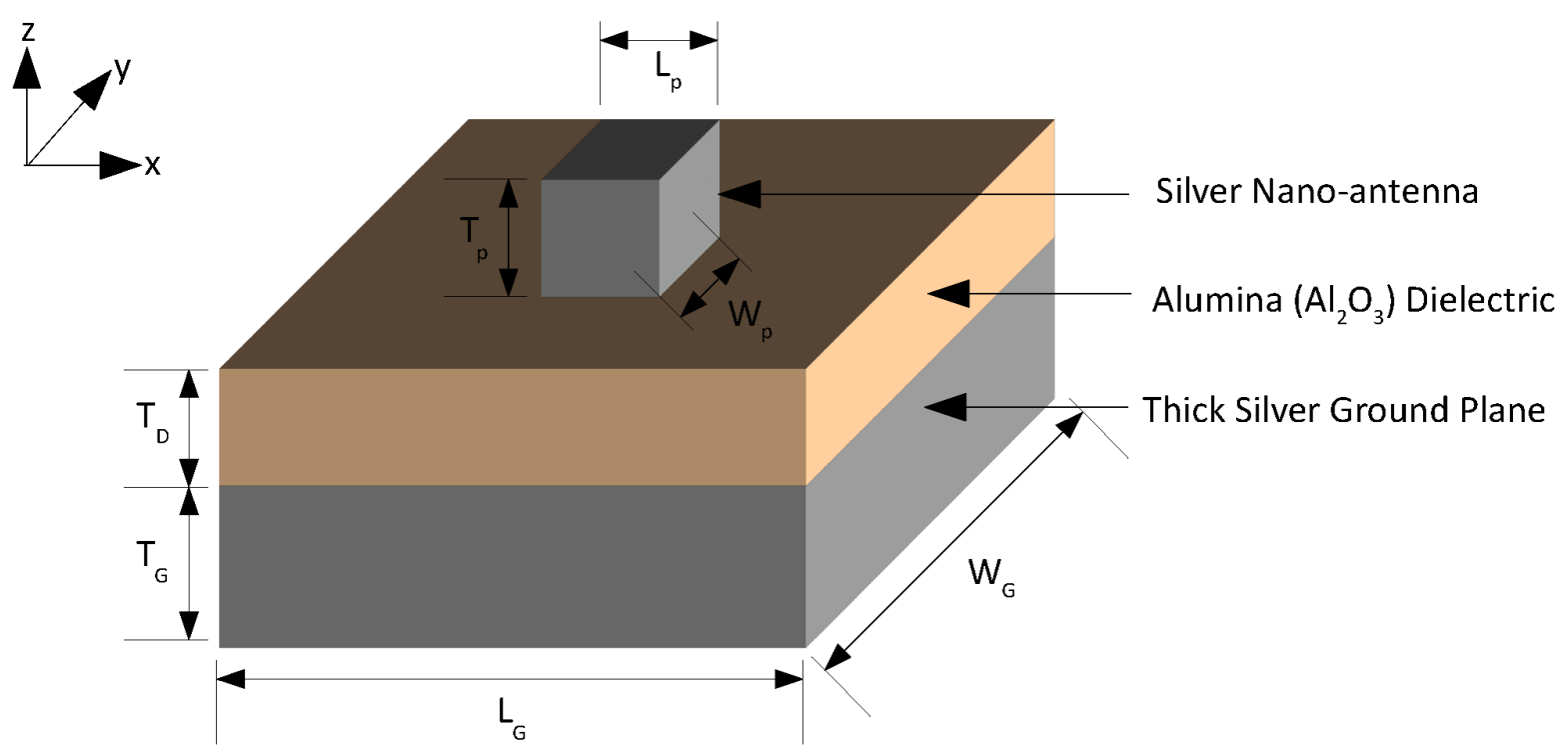

Figure 4.9 Dimensional illustration of the metamaterial nano-antenna constructed from a nanoparticle separated from a ground plane with an alumina dielectric. Critical parameters to this research are the particle width $\left(W_{p}\right)$ and dielectric thickness $\left(T_{D}\right)$. The metamaterial composite is perfectly symmetrical is infinite in the $\hat{x} \hat{y}$-plane.

As can be observed from Figure 4.10, the ground plane of the composite is constructed sufficiently thick enough to hinder any transmission through the cross section of the antenna. From Table 4.1, the metallic ground plane had a thickness of $80 \mathrm{~nm}$ which was considered thick enough to obstruct any transmission. 

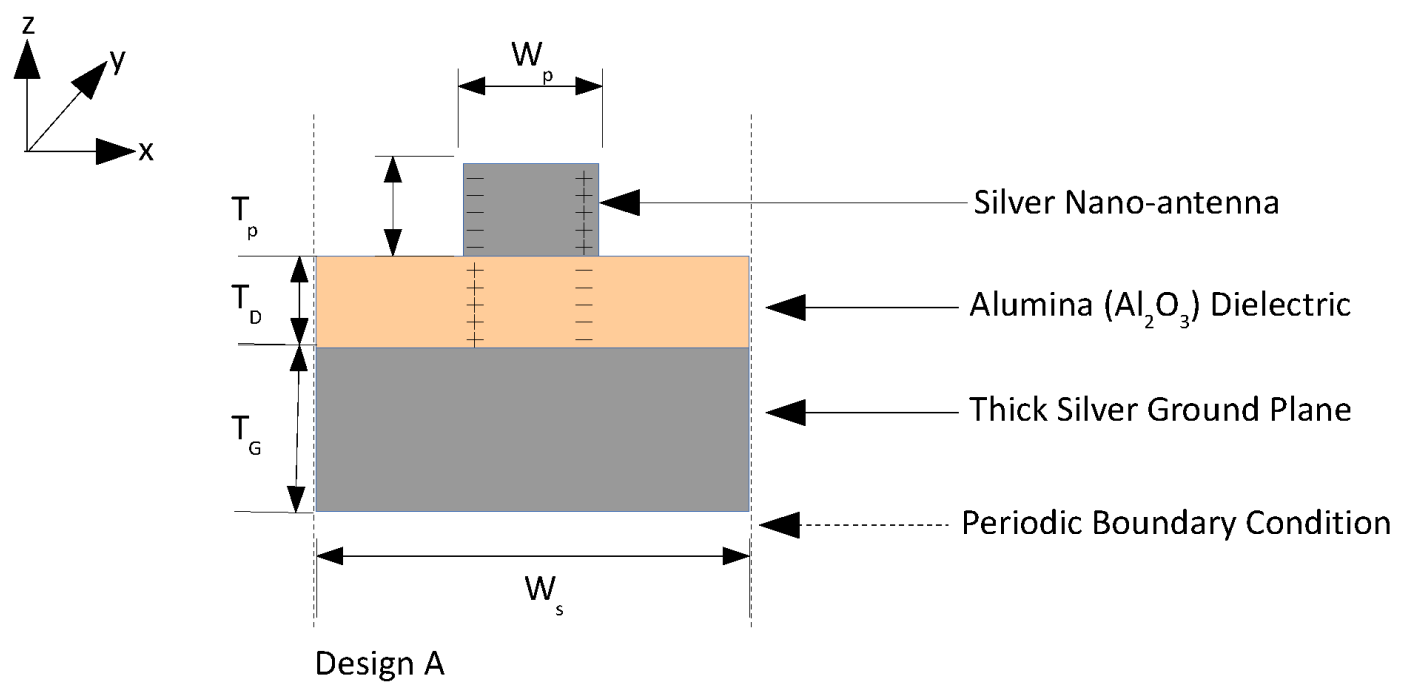

Figure 4.10 Front view illustration of the unit cell nano-antenna utilized as Desgin A containing all the parameters used in the composite construction.

\begin{tabular}{|c|c|c|c|}
\hline \hline Constituent & Parameter & Description & Parameter Value $(\mathrm{nm})$ \\
\hline \hline \multirow{3}{*}{ Nanoparticle } & $W_{p}$ & particle width & 50 \\
& $L_{p}$ & particle length & 50 \\
& $T_{p}$ & particle thickness & 30 \\
\hline \multirow{3}{*}{ Dielectric } & $W_{d}$ & dielectric width & 250 \\
& $L_{d}$ & dielectric length & 250 \\
& $T_{d}$ & dielectric thickness & 12 \\
\hline \multirow{3}{*}{ Substrate } & $W_{G}$ & substrate width & 250 \\
& $L_{G}$ & substrate length & 250 \\
& $T_{G}$ & substrate thickness & 80 \\
\hline
\end{tabular}

Table 4.1 Critical parameters used in the construction of a nano-particle sized unit celled antenna to study the interaction effects arising as a result of repeating boundary conditions.

\subsubsection{Resonance information for the first order absorber}

After the broadband sweep using the frequency domain solver, the simulation spectra showed an almost unity sharp absorption peak at $\omega_{0}=501 \mathrm{THz}$ as shown Figure 4.11. From Figure 4.12 c) it can be inferred that the absorption is largely due to the magnetic influence and therefore, the resonance frequency can be easily controlled without affecting the efficiency of the absorption peak by changing the dimensional parameters. 


\begin{tabular}{|l|c|c|}
\hline \hline Material & Plasma Freq. $\left(\omega_{p}\right)[\mathrm{rad} / \mathrm{s}]$ & Collision Freq $\left(\gamma_{c}\right)[\mathrm{rad} / \mathrm{s}]$ \\
\hline \hline Silver & $1.4 \mathrm{e} 16$ & $2.7 \mathrm{e} 13$ \\
\hline Alumina & $1.4 \mathrm{e} 16$ & $4.1 \mathrm{e} 13$ \\
\hline
\end{tabular}

Table 4.2 Dispersion parameters used in the Drude model for the nanoparticle (silver) and the dielectric (alumina).

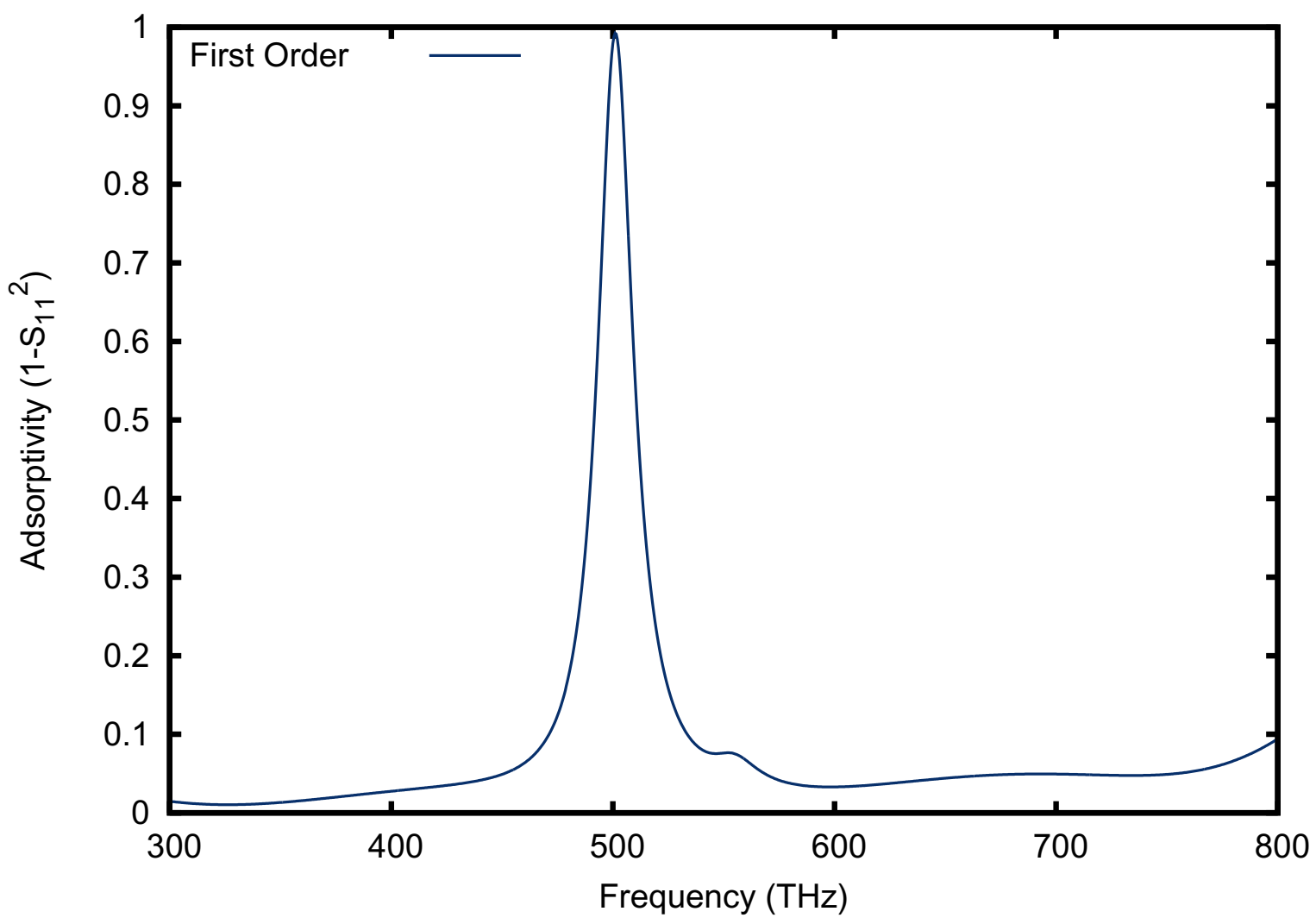

Figure 4.11 Absorptivity response of the silver nano-particle antenna of Design A as shown in Figure 4.10 with dimensions $W_{p} \times L_{p} \times T_{p}=50 \mathrm{~nm} \times 50 \mathrm{~nm} \times 30 \mathrm{~nm}$ and a dielectric thickness of $T_{D}=12 \mathrm{~nm}$ with electromagnetic radiation propagating in the - zdirection.

To interpret more clearly, the excitation mode is analyzed at the resonant peak by looking at the current distribution and electric and magnetic field distribution at that point, shown respectively in Figure 4.12 a), b) and c). Predominance of anti-parallel current as shown in Figure 4.12 a) is deemed responsible for the surface-plasmonic resonance induced 

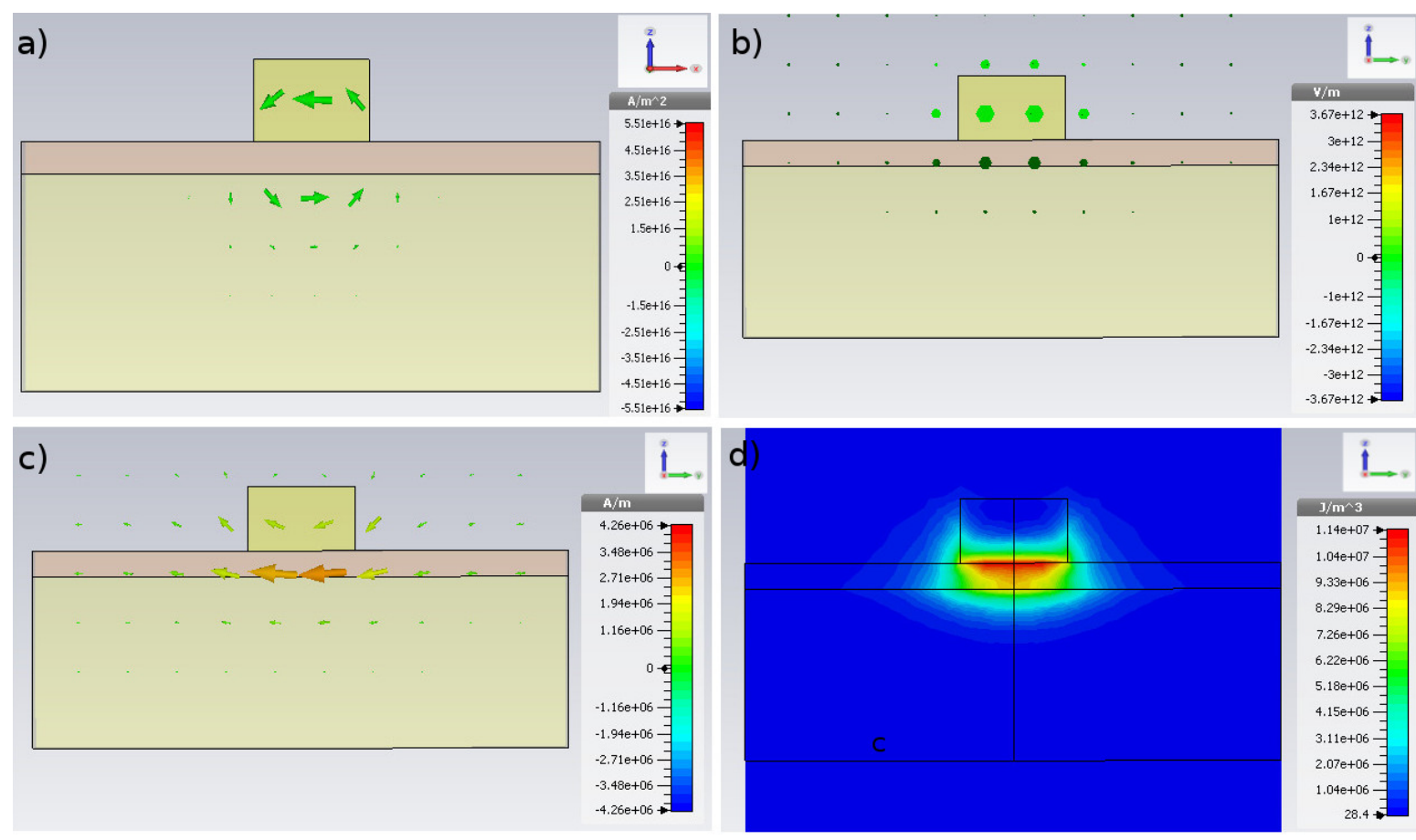

Figure 4.12 At resonant frequency $\omega_{0}=501 \mathrm{THz}$, a) Distribution of the induced (antiparallel) current. b) Electric field distribution. c) Magnetic Field Distribution. d) Distribution of the the power loss density (absorption).

by the external magnetic field. In addition, from Figure $4.12 \mathrm{c}$ ) it can be understood that the major electromagnetic energy is dissipated in the capacitors that would be created by the magnetic dipole mode inside the dielectric part inside the antenna . That is, the dielectric loss is dominant in achieving the sharp spectra of this simple antenna which supports the classic belief that the major power loss occurs in the dielectric parts which are the insulator elements of our metamaterial design. Figure 4.12 d) presents the power loss density confirming that all the electromagnetic radiation was absorbed in the dielectric media. Furthermore, it can be inferred that our single cell square shaped antenna posses the ability to concentrate electromagnetic waves in some specified positions. 


\subsubsection{Adaptive Mesh Refinement}

The simulation of metamaterials are very sensitive to the implementation of the "mesh cells" during the runs. Variations in mesh environment can give rise to slightly varying resonant frequencies which can affect the results. One effective way to overcome this hurdle is to use a technique called "adaptive mesh refinement" in the FDTD. In this technique, the s-parameters are calculated repeatedly with different mesh settings until the difference between the s-parameters is less than the specified value. Finally, it ensures that the mesh size is small enough to obtain stable results consistently. To evaluate the testing of meshing sensitivity on Design A, we ran the simulation with tetrahedral and hexagonal meshing and also using different number of mesh cells at the initiation of the simulation. The results are presented in Figure 4.13. Pass 1 represents the case where the mesh adaption function was not implemented. From Pass 1, it can be observed that even though the resonant frequency is in sync with the other passes, there is a slight discrepancy around $560 \mathrm{THz}$. Pass 4 presents the case where the simulation was conducted using a relatively large number of meshing cells and a more stable result was obtained (see Figure 4.11). From Figure 4.13, it can be observed that the resonant peak for this design is significantly insensitive to the sensitivity of the mesh cells, hence, for a quick design verification, this simulation can be performed without having to worry about the type and the number of meshing cells. 


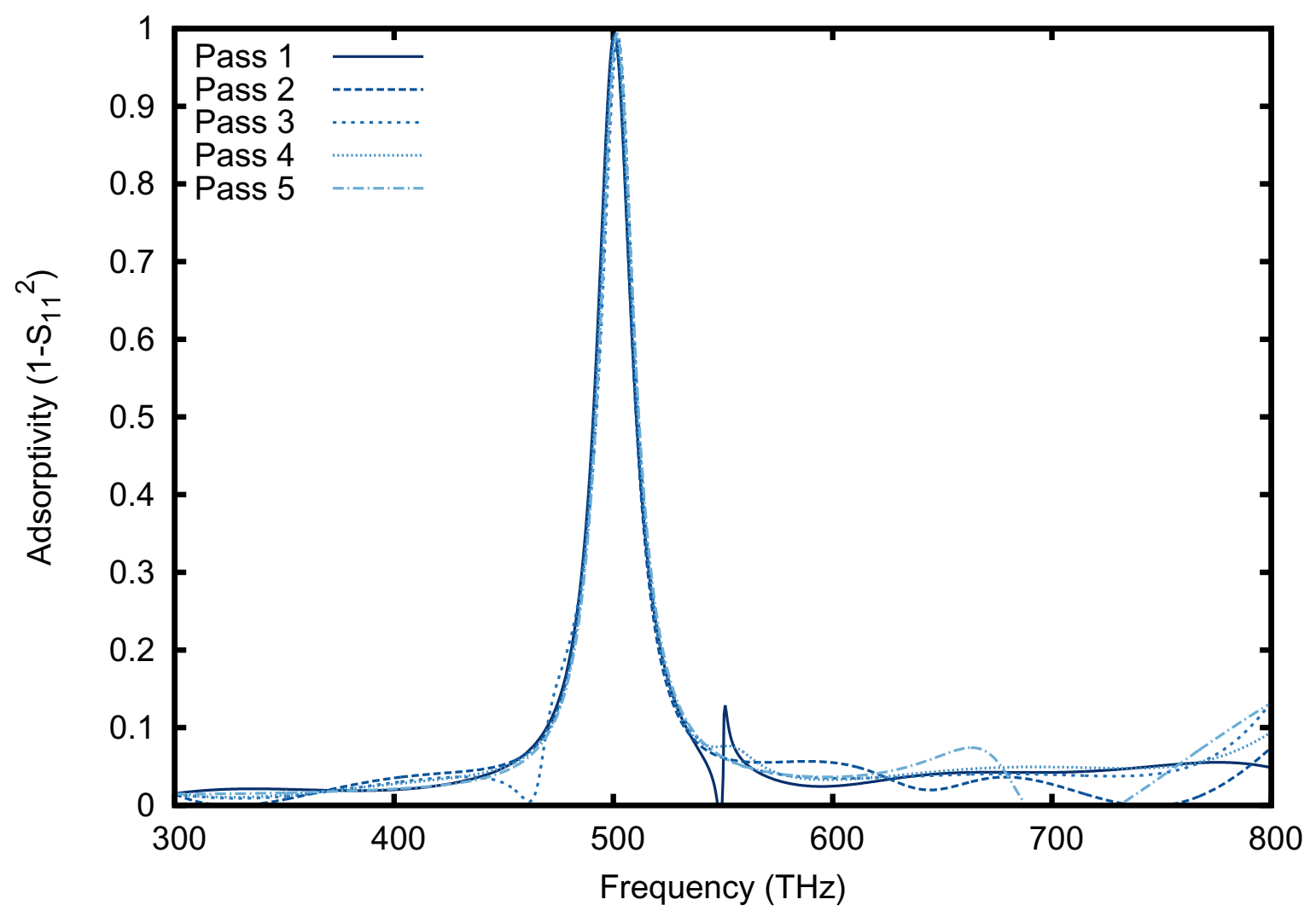

Figure 4.13 A comparison between different passes in the frequency domain solver of Design A as shown in Figure 4.10.

\subsubsection{Polarization Independence of the First Order Absorber}

Due to the perfectly symmetrical nature of our unit-celled design, one would intuitively say that the structure is independent of the polarization of the normal incident light. To confirm this notion, the same structure of Figure 4.9 was simulated for the TM and TE polarizations. The results are shown in Figure 4.14 which indicates that the absorption is independent of polarization. This is because the orientation of the magnetic field of the normal incident radiation does not change. Hence as discussed in Section 4.2.2, the influence of magnetic resonance remains dominant. 


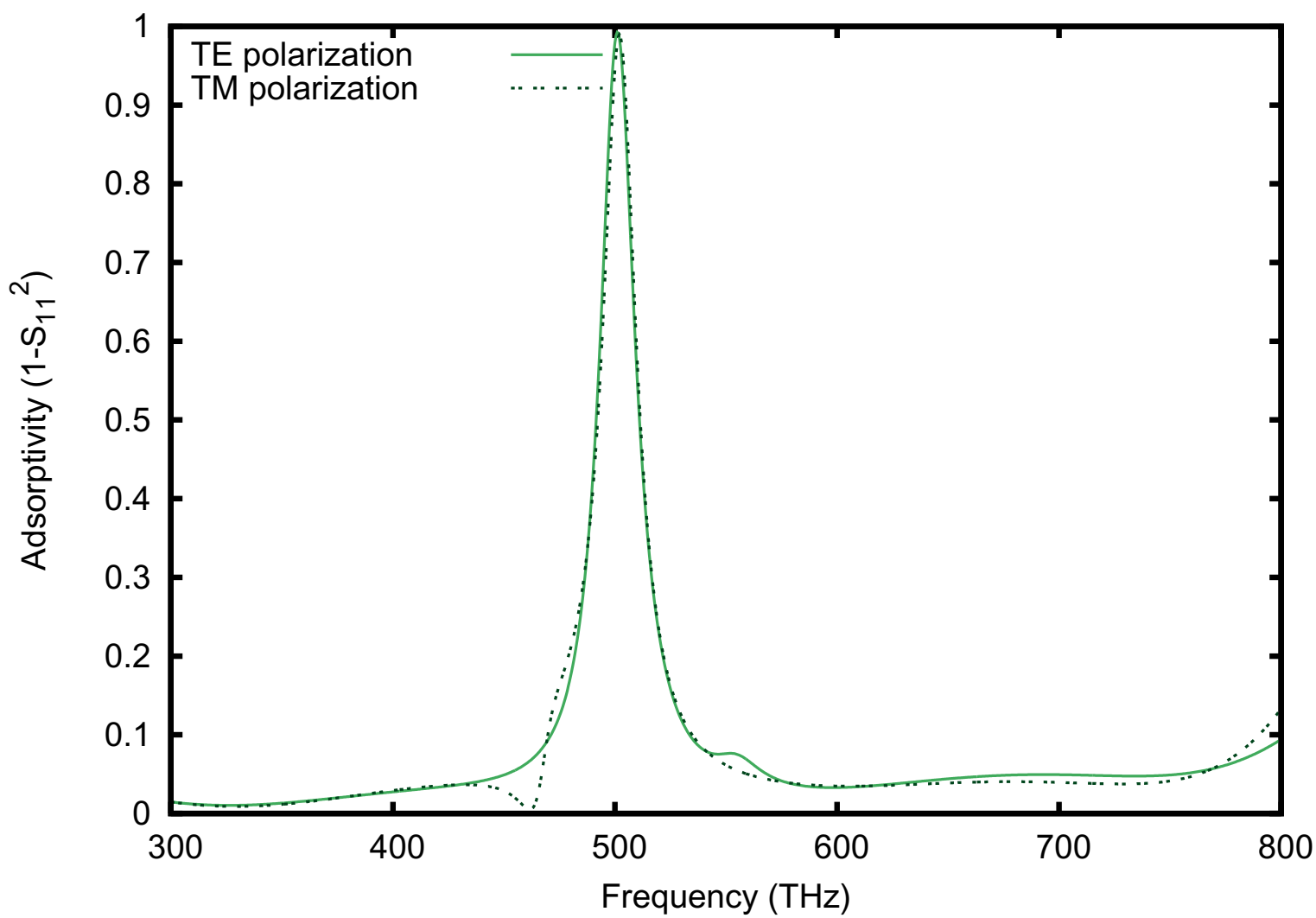

Figure 4.14 A comparison between TE and TM polarization on the absorption effect of the First Order Absorber of Design A as shown in Figure 4.10.

\subsubsection{Comparison of different unit-celled nano-antennas}

In order to further investigate the effect of geometry of the nano-particle antenna on the absortion, three different unit structures were constructed and simulated in the similar way as discussed in Section 4.2.1. Figure 4.15 illustrates the three different designs that were considered as the preliminary unit cell antenna a) square, b) diamond and c) a cylindrical shaped respectively. The results, as presented in Figure 4.16, indicate that for the diamond and the square scenario, the is no visible shift in the fundamental resonant mode. From this, it can be inferred that the orientation of the square nano-antenna does not have any significant effect on the absorptivity. However, of importance to note is the second and third resonant modes at the higher end of the spectrum. This is attributed to the sharp edges of 
the diamond shape which induces electromagnetic waves to be concentrated at that specified location. The cylindrical shaped nano-antenna absorbs much significantly at the lower end and at the far end of the spectrum which is actually outside the region of interest, that is, the visible regime. For this reason, the cylindrical design was abandoned as a potential Design A antenna.
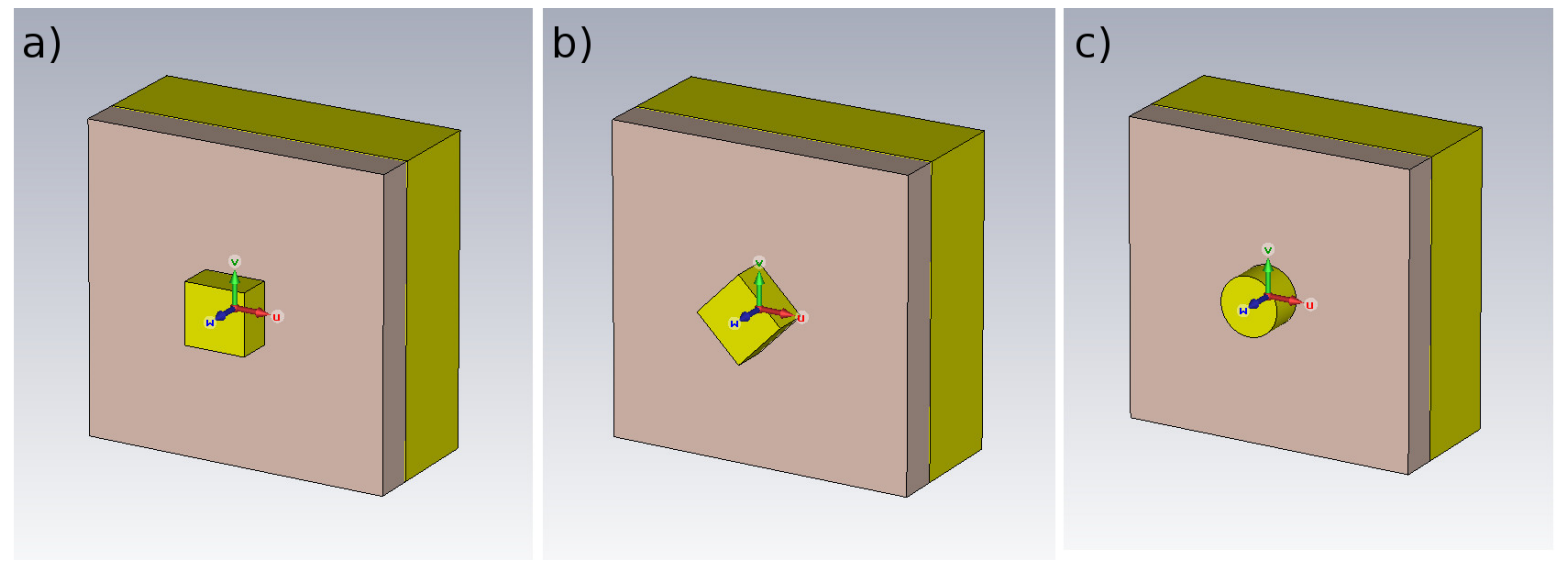

Figure 4.15 Frequency response of three different unit-celled nano-antennas to observe the influence of geometry on the absorption response. 


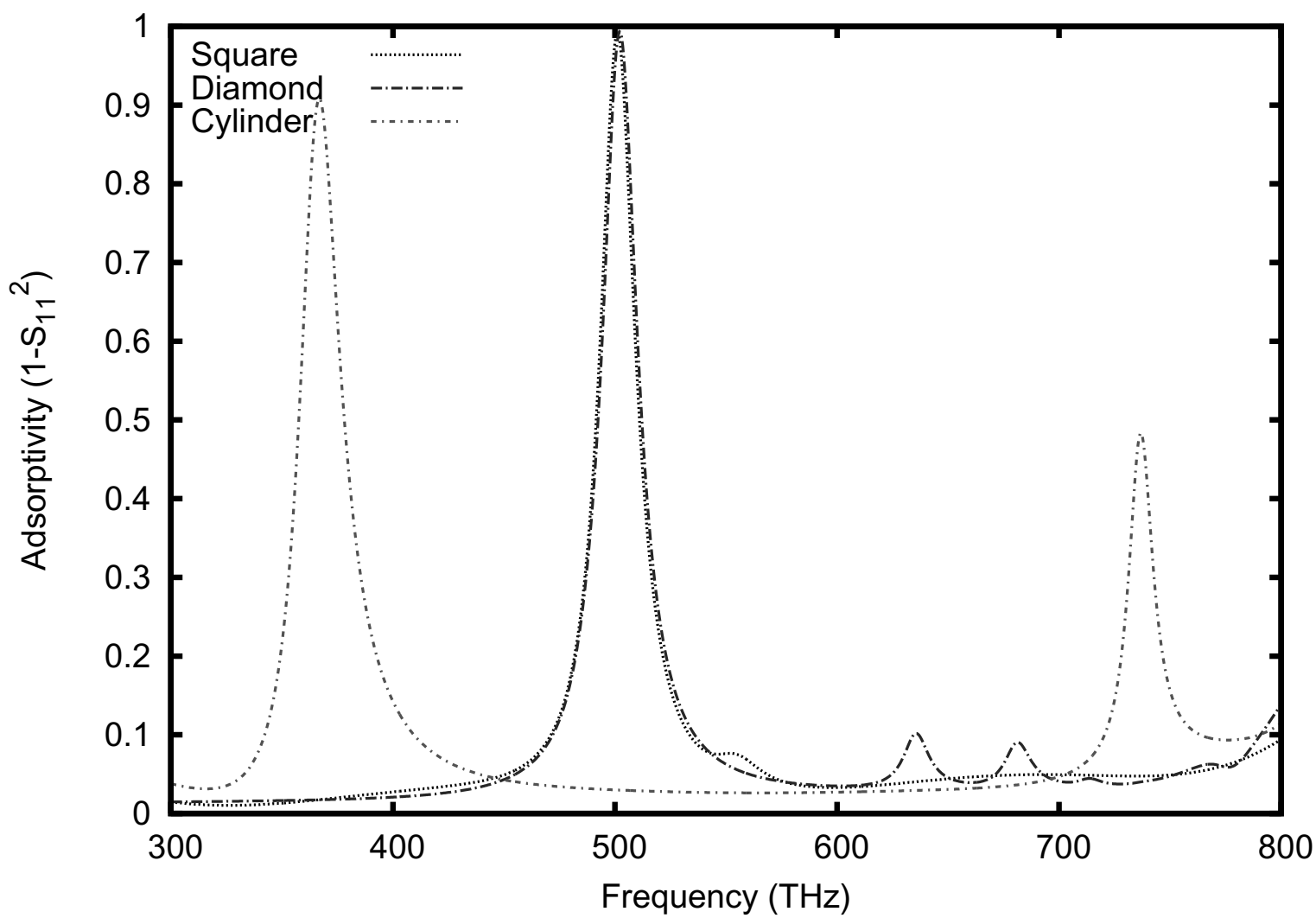

Figure 4.16 The electromagnetic response in terms of absorption for three different unit cells as presented in Figures 4.15 a), b) and c).

\subsection{Influence of nano-particle width and dielectric thickness}

Having understood the effect of magnetic resonance as the major contributor on the absorptivity of our unit-celled solar antenna, the attention shifted to predicting the absorptivity as a function of geometry by investigating a two parameter design space. More specifically, to understand the influence of the dielectric thickness and particle width on the electromagnetic response of our structure. This approach was undertaken to determine accurately the frequency dependence of the dielectric media as well as the geometry of the silver nano-antenna. Emphasis on this simulation was focused on the frequency response as a function of both the particle width and dielectric thickness.

A parameter sweep, of the same design as presented in Figure 4.9, of the particle width 
$\left(W_{p}\right)$ and the dielectric thickness $\left(T_{d}\right)$ was calculated and the absorptivity was calculated at each configuration. The results are shown in Figure 4.17 illustrate the response of the peak absorption as a function of the two geometry parameters.

A intriguing feature of this figure is the bifurcation of the response with increasing particle width as illustrated in Figure 4.17. At a particle width of approximately 40nm, the bifurcation originates resulting in two peak absorption that is dependent on the dielectric thickness.

The two absorption peaks that comprise the bifurcation can be visualized more clearly in Figure 4.18, which is a 2D slice of Figure 4.17 at a fixed particle width of $80 \mathrm{~nm}$. Figure 4.18 (a) and (b) represents the reflectivity and absorptivity respectively.

This response can be reasoned based on Equation 2.27, where the polarizability or the ability to form plasmons dependent on the damping $(\gamma)$ of the materials. This damping term is ultimately related to the complex effective dielectric constant for the dielectric material. As a results, as the dielectric thickness increases, the effective dielectric constant changes. Therefore, the lower peak in Figure 4.18(b) is the first resonance at a dielelectric thickness of $25 \mathrm{~nm}$ and the second peak at a dielectric thickness of $60 \mathrm{~nm}$ is the second resonance. The second resonance is slightly shifted in frequency because the polarizability has an imaginary component.

Referring back Figure 4.17, a 2D slice can be taken where the dielectric is held constant at $50 \mathrm{~nm}$, slicing through one of the bifurcation legs. The results of this slice are shown in Figure 4.19 as (a) the reflectivity and (b) the absorptivity. The defining feature of this figure is the lack of correlation between the particle width and frequency response at large dielectric thicknesses. For a 50nm dielectric thickness, when the particle width is increased, the response is moderately constant with a peak absorption at $525 \mathrm{~nm}$. However, the peak response does not originate until the particle width is about one tenth $(60 \mathrm{~nm})$ the peak absorption wavelength $(600 \mathrm{~nm})$. From Figure 4.17, as the particle width increases the slope of both bifurcated legs approach zero demonstrating the eventual independence of the particle 
width on the response.

\section{Normailzed Absorptivity (a.u.)}

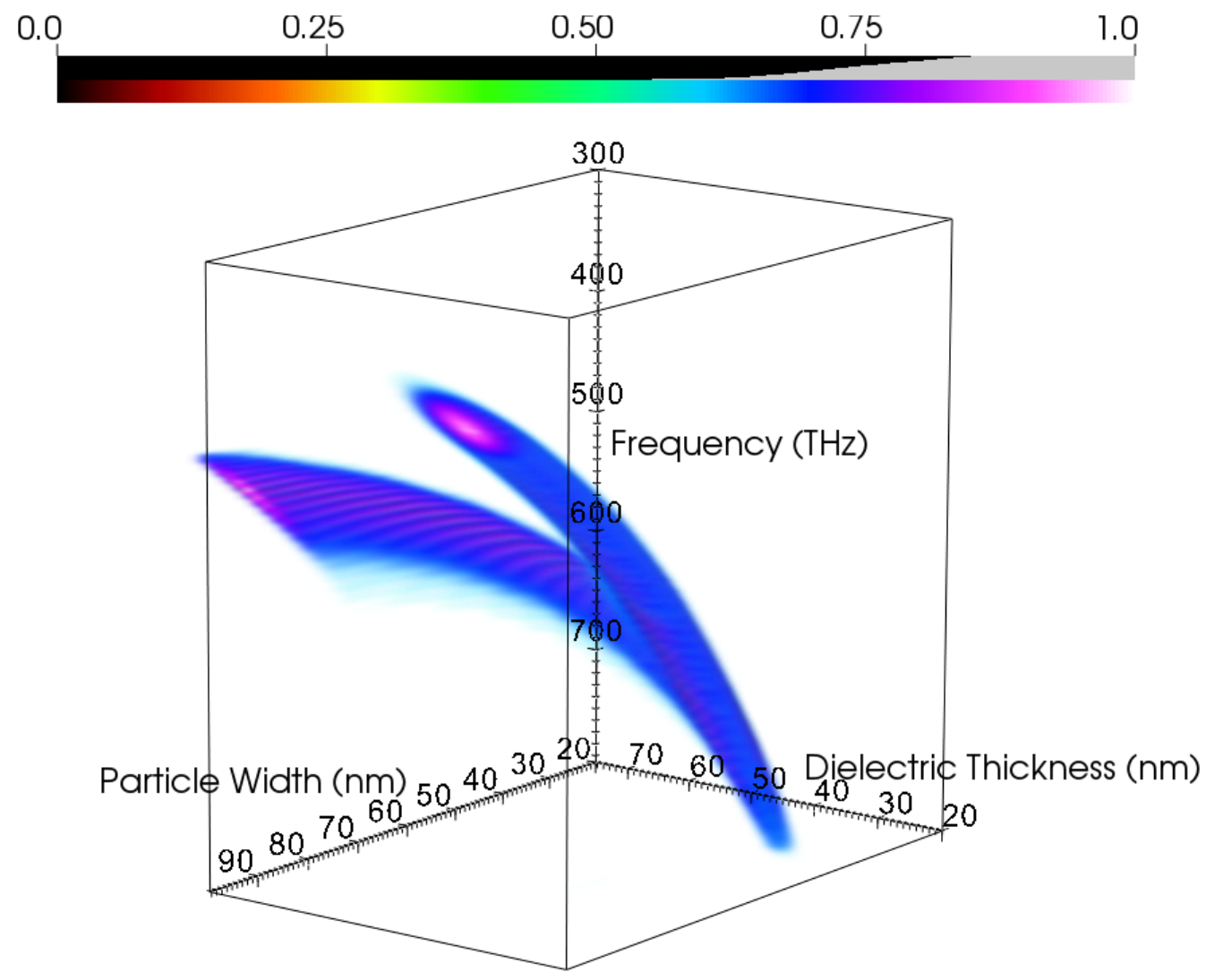

Figure 4.17 Plot of the normalized absorptivity as a function of both the particle width $\left(\mathrm{W}_{p}\right)$ and the dielectric thickness $\left(T_{d}\right)$. The figure illustrates a bifurcation of the peak absorption with a decrease in frequency. This figure can aid in determining geometric aspects of a $\mathrm{Al}_{2} \mathrm{O}_{3}$ dielectric-silver composite metal materials (see Figure 4.9 for construction). 

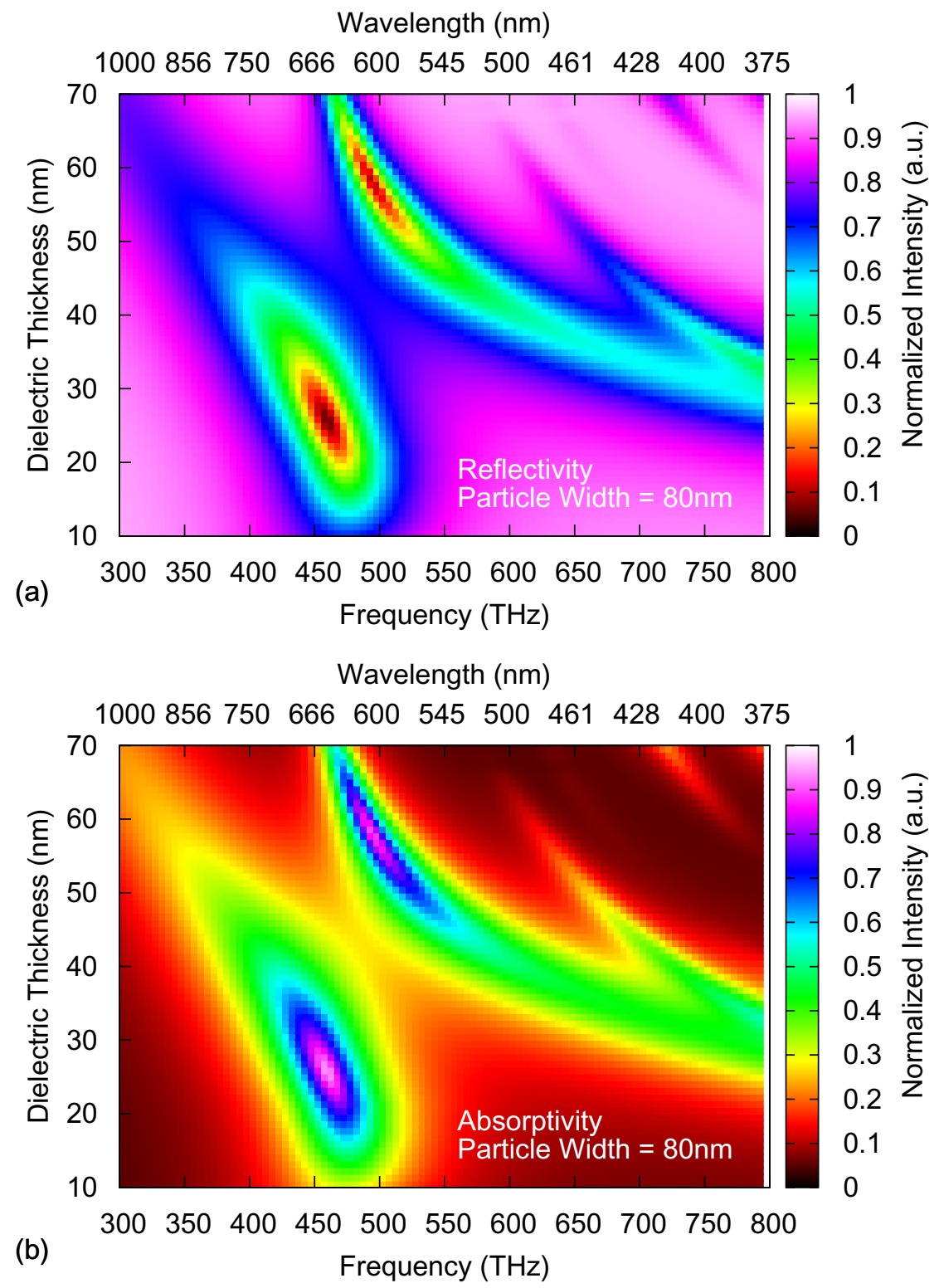

Figure 4.18 Plot of the frequency response versus dielectric thickness for a $\mathrm{W}_{p}=80 \mathrm{~nm}$ particle. Figure (a) is the reflectivity and figure (b) is the absorptivity. There are two peak aborption regions. The first mode is at a dielectric thickness of $25 \mathrm{~nm}$ and the second mode is at a dielectric thickness of $60 \mathrm{~nm}$. 


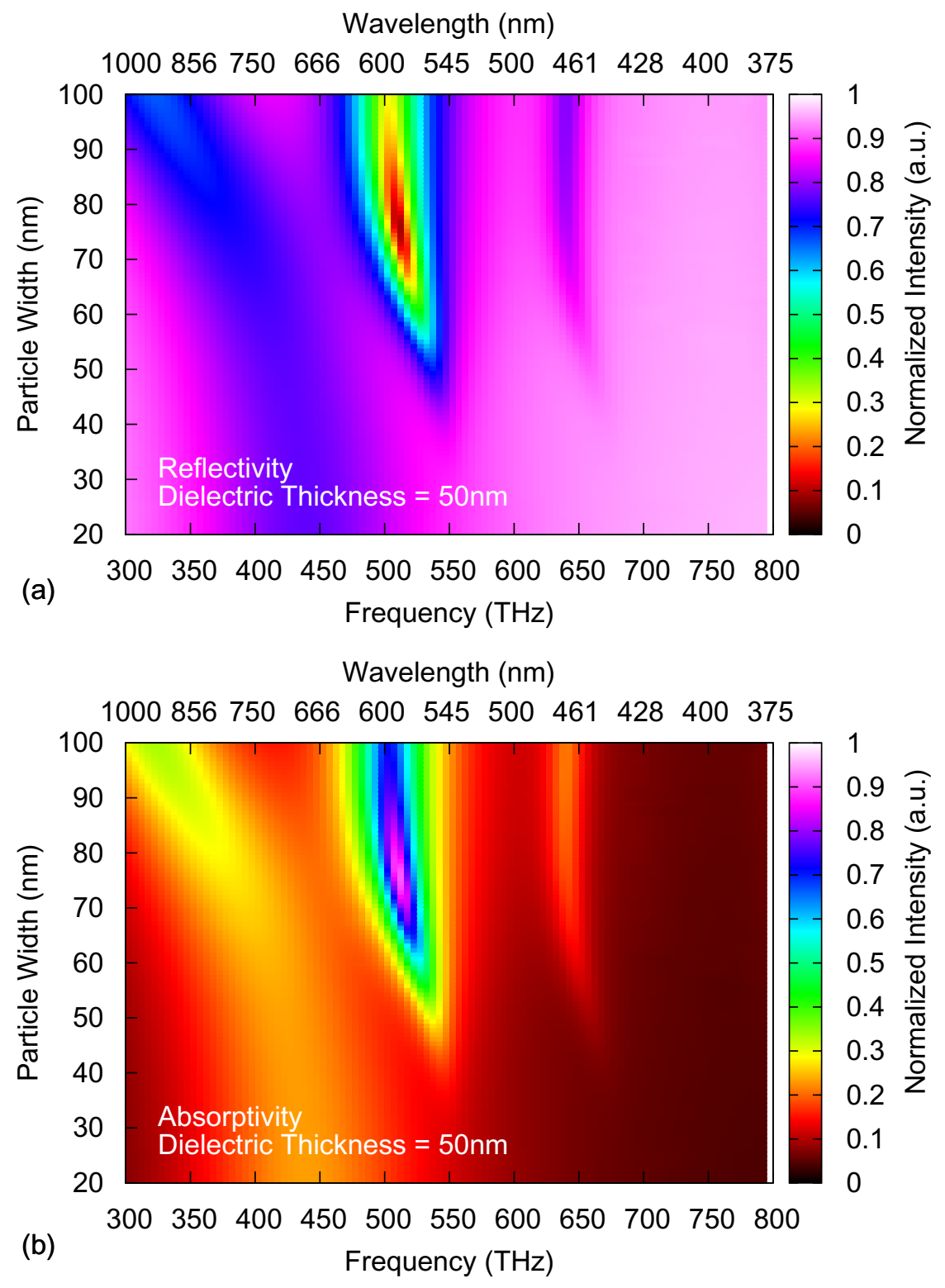

Figure 4.19 Plot of the frequency response versus particle width. The dielectric thickness $\mathrm{T}_{d}=50 \mathrm{~nm}$ and the particle is assumed square. Figure (a) is the reflectivity response and Figure (b) is the absorptivity. Peak absortion is peak at a particle size of $75 \mathrm{~nm}$. 


\subsection{Interaction effects due to neighboring particles}

From the macroscopic point of view, this simple nano-antenna can be visualized as an array of nano-antennas. This effect is a result of neighboring particles as presented in Figure 4.20 and 4.21. From Design A, provided that the geometries are tuned appropriately, the outcome is a singular frequency with perfect absorption. Therefore, if it was possible to take this understanding a step further and include multiple particles and understand the influence of additional particles on the frequency response, the response could potentially be broadened to encompass several frequencies. This research is aimed at determining this complex interaction of neighboring particles. These neighboring particles each posse their own electromagnetic response which interferes with the surrounding responses. In particular, it becomes imperative to understand the influence of the neighboring nano-antennas on the total electromagnetic response of our composite structure.

A parameter sweep, of the same design as presented in Figure 4.9, was conducted for the particle spacing from 75 to $500 \mathrm{~nm}$ and the dielectric thickness from 5 to $33 \mathrm{~nm}$. The absorptivity was calculated at each configuration. Emphasis on this simulation was focused on the frequency response as a function of both the particle spacing and dielectric thickness. The parameters for this investigation are presented in Table 4.3. Figure 4.22 shows three different categories of interactions arising from neighboring particles 1) cavity interaction 2) effective medium interaction and 3) grating coupling design in addition to the anti-parallel current interaction (Design A) as explained in Section 4.2.2. These are all derivations from

the array arrangement from Figure 4.21. The results are presented in Figures 4.23, 4.27 and 4.28. 


\begin{tabular}{|c|c|c|c|}
\hline \hline Constituent & Parameter & Description & Parameter Value $(\mathrm{nm})$ \\
\hline \hline \multirow{3}{*}{ Nanoparticle } & $W_{p}$ & particle width & 75 \\
& $L_{p}$ & particle length & 75 \\
& $T_{p}$ & particle thickness & 30 \\
\hline \multirow{3}{*}{ Dielectric } & $W_{d}$ & dielectric width & 500 \\
& $L_{d}$ & dielectric length & 500 \\
& $T_{d}$ & dielectric thickness & 12 \\
\hline \multirow{3}{*}{ Substrate } & $W_{s}$ & substrate width & 60 \\
& $L_{s}$ & substrate length & 60 \\
& $T_{s}$ & substrate thickness & 150 \\
\hline
\end{tabular}

Table 4.3 Critical parameters used in the construction of a nano-particle sized unit celled antenna to study the interaction effects arising as a result of neighboring particles.

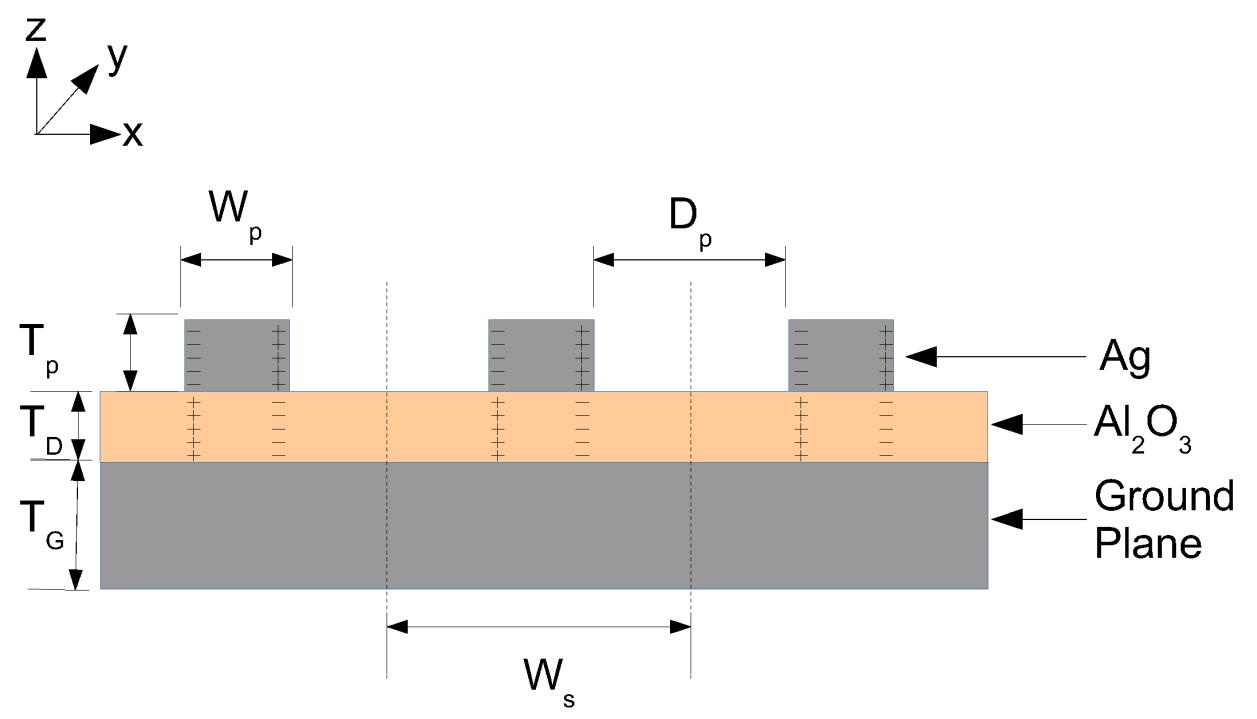

Figure 4.20 A two dimensional illustration of the unit cell computational domain and the critical dimensions. Interaction between neighboring particles was handled through symmetry boundary conditions at $\mathrm{x}$-min and $\mathrm{x}$-max. The actual computational domain used in FDTD calculation was 3D dimension, where the 3rd dimension is equivalent to the $\mathrm{x}$ dimension. 


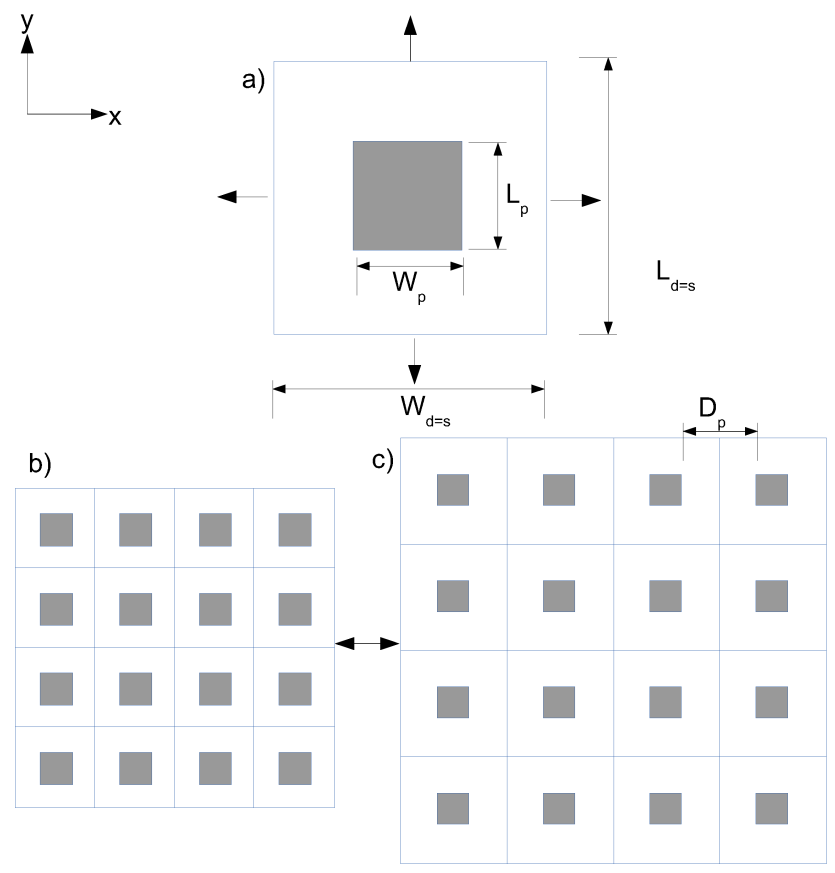

Figure 4.21 Illustration of Figure 4.20 as an array arrangement to study the interaction effects with changing particle spacing.

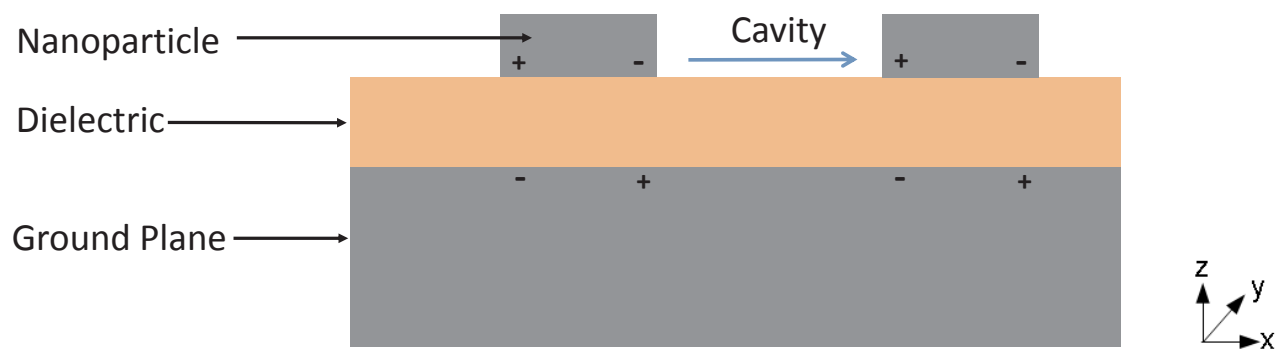

Design B

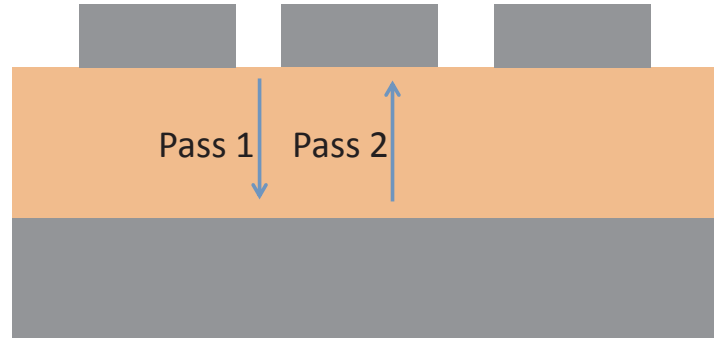

Design C

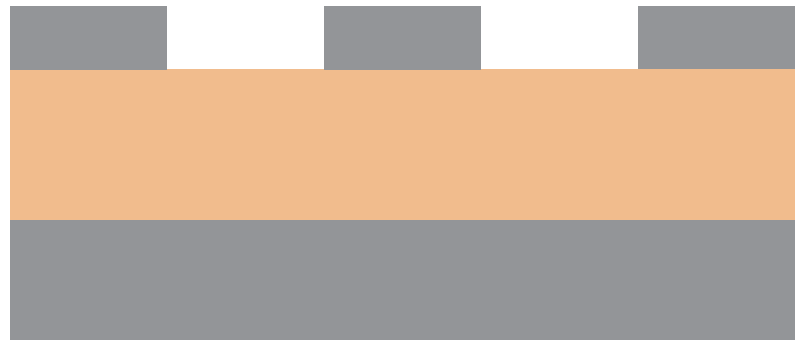

Design D

Figure 4.22 Illustration of the three dominant interactions that are responsible for the response resulting from an array arrangement. Design B is a cavity interaction between particles. Design C is an effective medium design. Design D is a grating coupling design. 
The second design involves forming a cavity between the neighboring particles. This corresponds to Design B in Figure 4.22. The cavity response of this design is analogous to that of a nanohole array where the incident electromagnetic wave resonates between the particles. Because of the proximity of the closely spaced silver nano-particles, extreme coupling occurs. This coupling leads to strong field enhancement in the near field, thus enhancing the light-matter interaction and resulting in perfect absorption with multiple resonances. Figure 4.23 are select runs where the distance between the particles decreases, that is, with increasing particle size. As this distance decreases, the response red shifts as also illustrated in Figure 4.24. All the fundamental modes of the respective runs are shifting monotonously with increasing particle size towards smaller frequency. This can be conceptually thought of as longer wavelengths coupling within larger hole diameters. Also, worth noting is that the dielectric thickness does not influence the response as illustrated by the selected points.

Moreover, it can be seen that as the particle width increases, the excitation strength is enhanced and the absorption is increased. To further illustrate this point, Figure 4.24 presents three different cases at a constant dielectric thickness of $12 \mathrm{~nm}$. The fundamental mode appears at $\omega_{1 s t}=648.5 \mathrm{THz}$, the second resonant mode appears at $\omega_{2 n d}=721.5 \mathrm{THz}$ and the third resonant mode appears at $\omega_{3 r d}=344 \mathrm{THz}$ for $D_{p}=10 \mathrm{~nm} . D_{p}=\left(W_{s}-W_{p}\right)$ where $D_{p}$ is the separation distance between two neighboring particles as presented in Figure 4.21. To compare, the change in absorption strength with increasing particle width, the bandwidth at absorption $=0.5$ for the three fundamental modes for their respective separation distances in considered. The results are presented in Table 4.4 from which it can be seen that there is a significant increase in the bandwidth with increasing particle width.

In addition, it can be noticed that the excitation strength of the modes decreases with increasing order of resonant modes, that is, with each successive resonant modes. For the case $D_{p}=10 \mathrm{~nm}$, there is a one third decrease in the absorption at $\omega_{3 r d}$ when compared to the $\omega_{1 s t}$ and $\omega_{2 n d}$. This is because as the separation distance between the two neighboring cells $\left(D_{p}\right)$ 


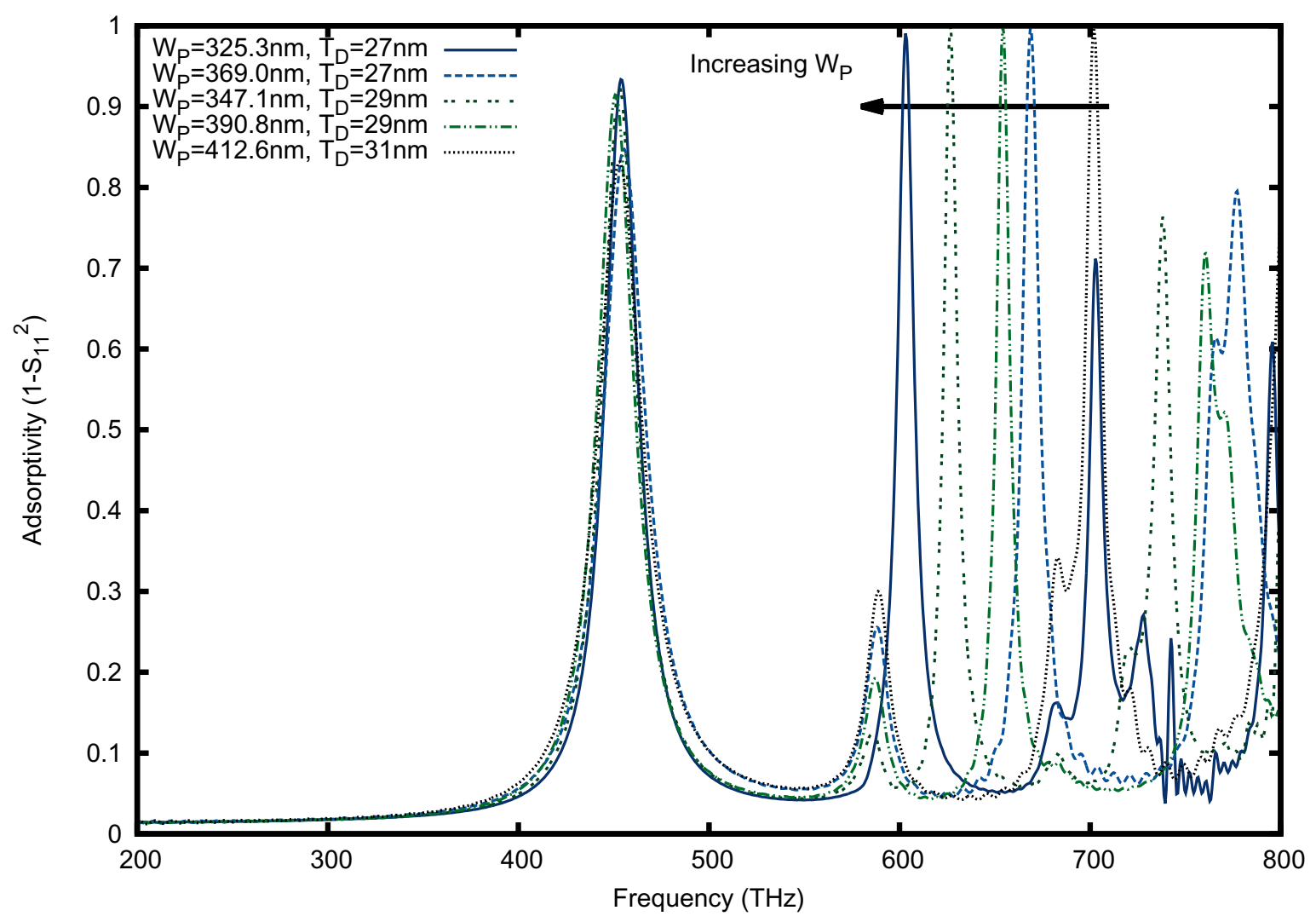

Figure 4.23 Results from Design B of an cavity response between particles resulting in perfect absorption. As the cavity increases the response blue shifts.

increases, the overlap (coupling effect) of the electromagnetic plane wave is reduced at each mode, therefore, reducing the hybridization effects. For higher order $n t h$ modes, that is, with extremely large $D_{p}^{\prime} s$ the overlap of the electromagnetic plane wave at normal incidence completely vanishes for $n$ tending to infinity. As such the the absorption response takes the characteristic as that of the isolated unit-celled square nano-antenna, that is, the first order absorber at $D_{p}=200 \mathrm{~nm}$. 


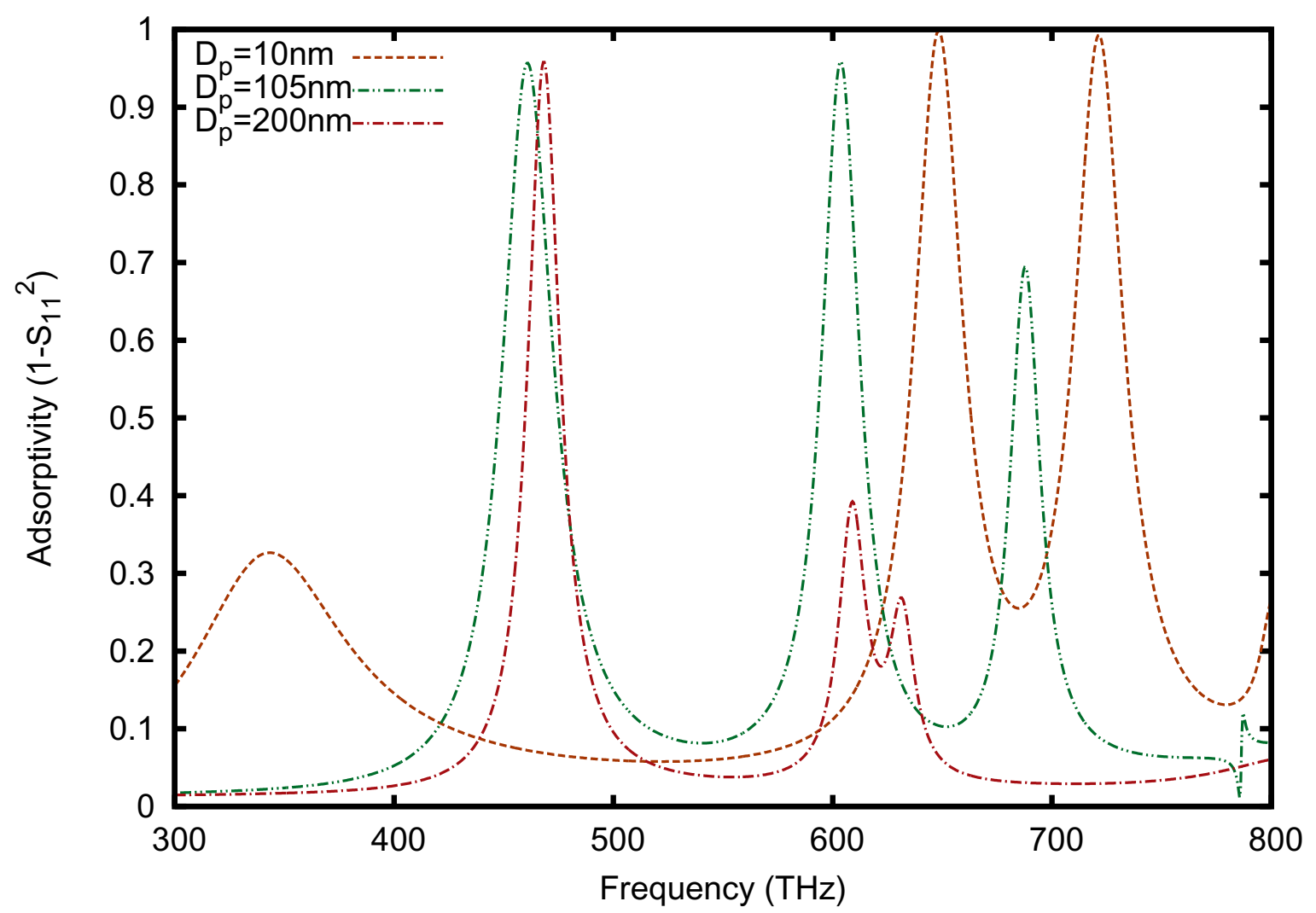

Figure 4.24 Interaction effects with increasing separation distance between unit cell. The dielectric thickness is held constant at 12nm of Design B as shown in Figure 4.22.

\begin{tabular}{|c|c|c|}
\hline \hline$D_{p}$ & Resonant Modes & Bandwidth at absorption $=0.5$ \\
\hline \hline \multirow{3}{*}{$D_{p}=10 \mathrm{~nm}$} & $\omega_{1 s t}$ & 16 \\
& $\omega_{2 n d}$ & 12 \\
& $\omega_{3 r d}$ & $\mathrm{NA}$ \\
\hline \multirow{3}{*}{$D_{p}=105 \mathrm{~nm}$} & $\omega_{1 s t}$ & 15 \\
& $\omega_{2 n d}$ & 11.5 \\
\hline \multirow{3}{*}{$D_{p}=200 \mathrm{~nm}$} & $\omega_{3 r d}$ & 5 \\
& $\omega_{1 s t}$ & 10 \\
& $\omega_{2 n d}$ & $\mathrm{NA}$ \\
& $\omega_{3 r d}$ & $\mathrm{NA}$ \\
\hline
\end{tabular}

Table 4.4 A comparison of the bandwidth at each resonant modes for successive separation distances $D_{p}^{\prime} s$ of the neigboring cells. 


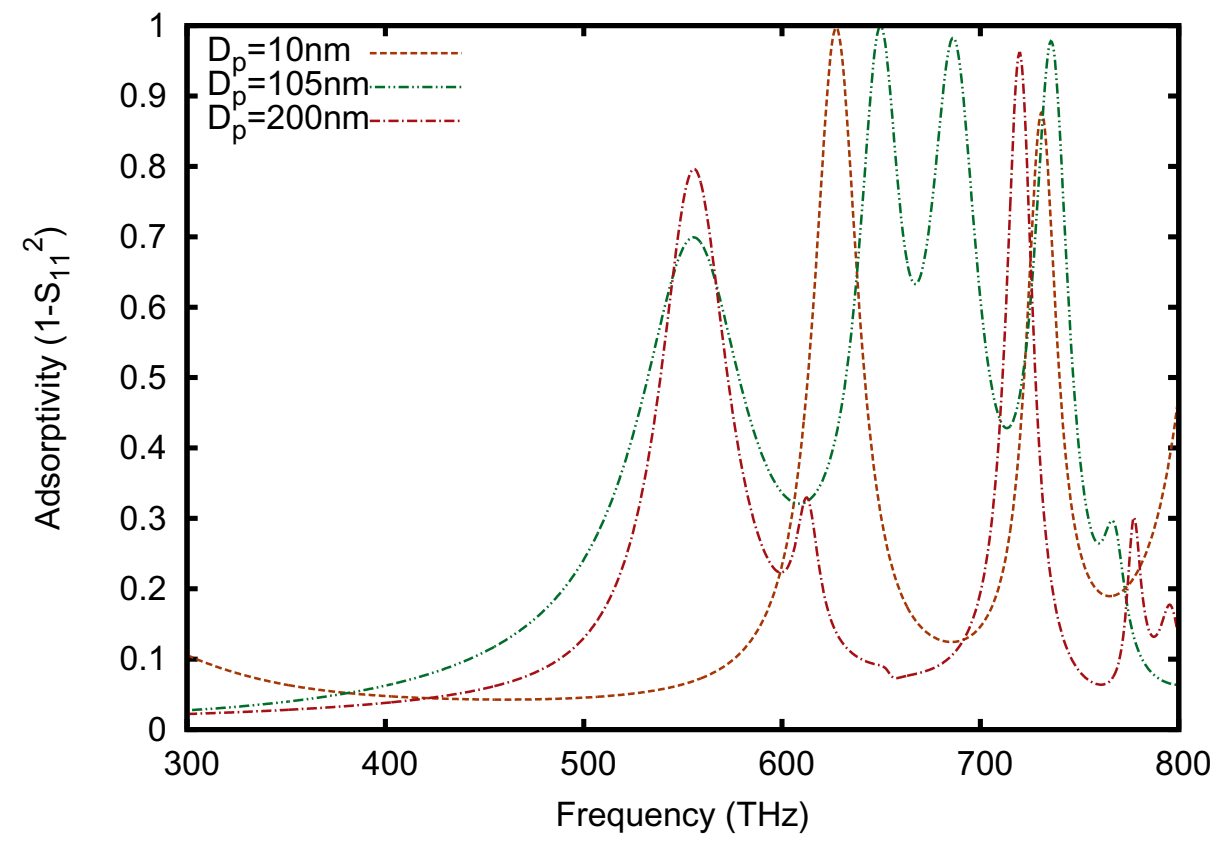

Figure 4.25 Interaction effects with increasing separation distance between unit cell. The dielectric thickness is held constant at 56nm of Design B as shown in Figure 4.22.

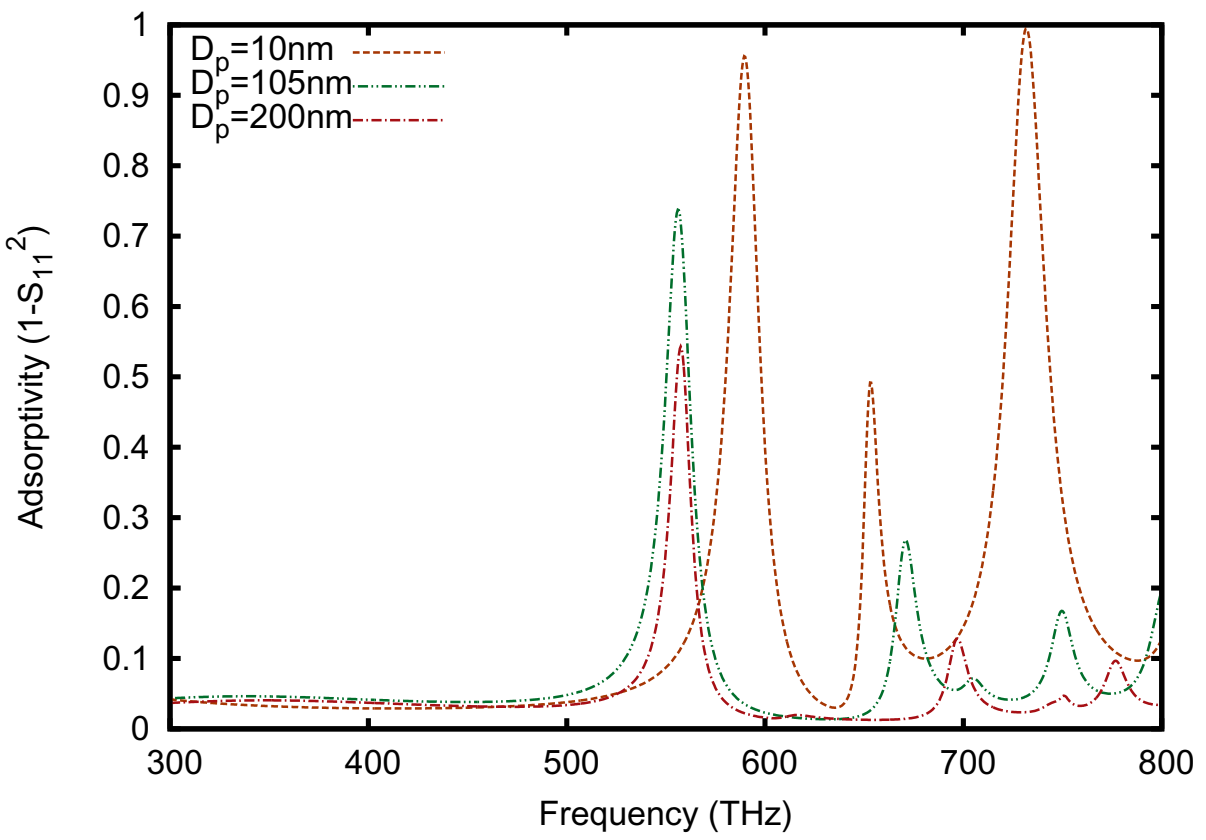

Figure 4.26 Interaction effects with increasing separation distance between unit cell. The dielectric thickness is held constant at 100 of Design B as shown in Figure 4.22. 
The third design is illustrated in Figure 4.22 (Design 3). It is based on an effective medium theory. This theory is based on the presumption that the particles situated on top of the dielectric act as a singular material with material properties that depend on the volume fraction of silver and air. As the distance between the particles increases the volume fraction of air increases. Perfect absorption can be obtained by impedance matching the effective medium with the dielectric. A resonance of $768.5 \mathrm{THz}$ occurs when the period is $85 \mathrm{~nm}$ (a 10nm distance between particles) and the dielectric thickness is greater than $19 \mathrm{~nm}$.

To impedance match the effective medium with the dielectric, the ground plane was removed and a set particle geometry was simulated. The peak frequency with a reflection of zero mark the impedance matched at $768.5 \mathrm{THz}$. Once this peak impedance match frequency was known the ground plan was put back in the simulation. The width of the domain was restricted in the width to $85 \mathrm{~nm}$ and the length was varied until the equivalent peak was found. The arrangement that demonstrated this similar response with the ground plane was a 85 by $85 \mathrm{~nm}$. This configure resulted in the correct volume fraction of air and silver for the corresponding permitivity of air. A series of runs illustrate this in Figure 4.23. The response of this effective medium requires that the dielectric thickness be greater than $19 \mathrm{~nm}$ to facilitate a long enough traverse time to absorbed the energy. 


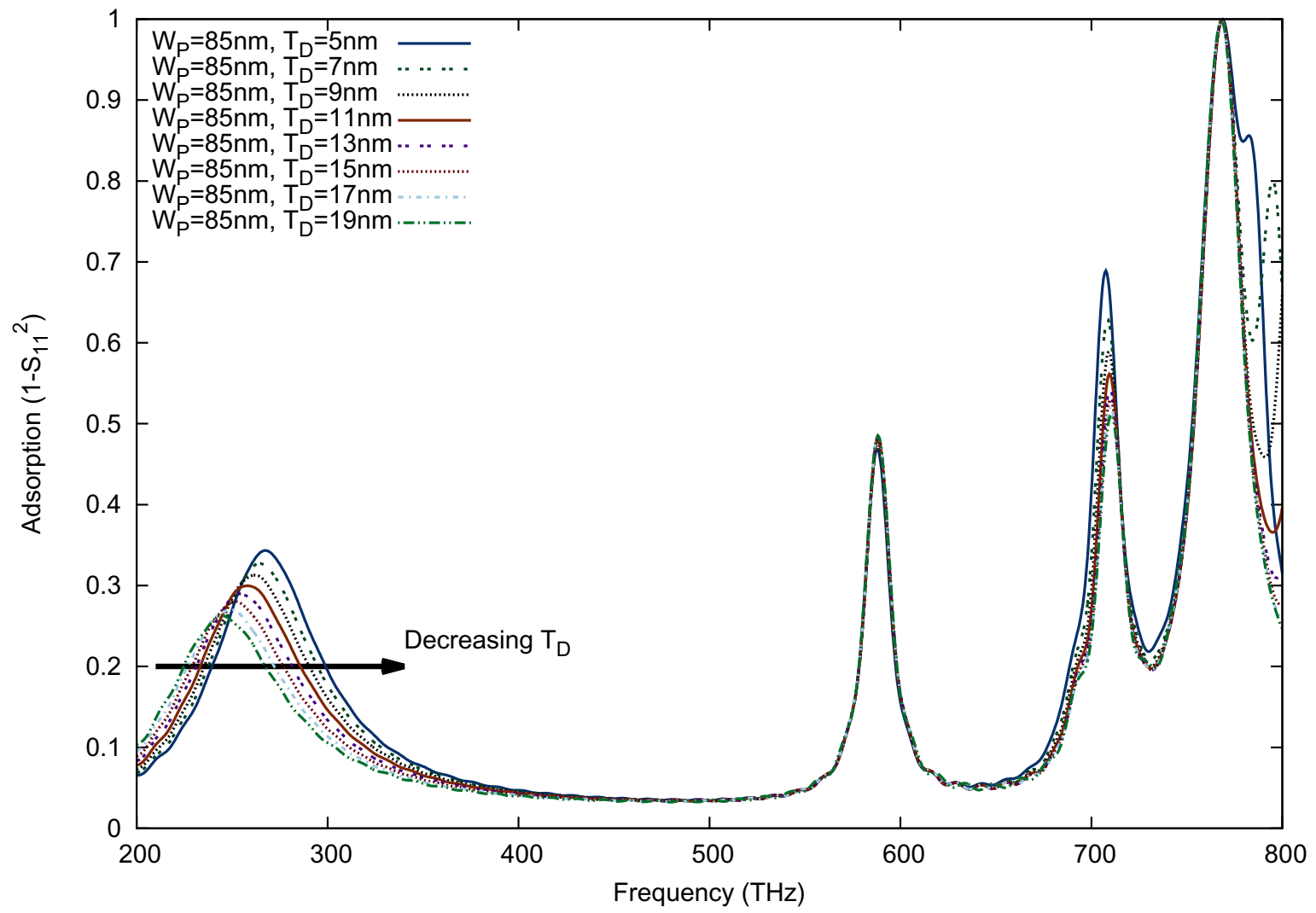

Figure 4.27 Results of Design $\mathrm{C}$ as an effective medium material where the volume fraction of the silver and air impedance match with the surrounding air. 


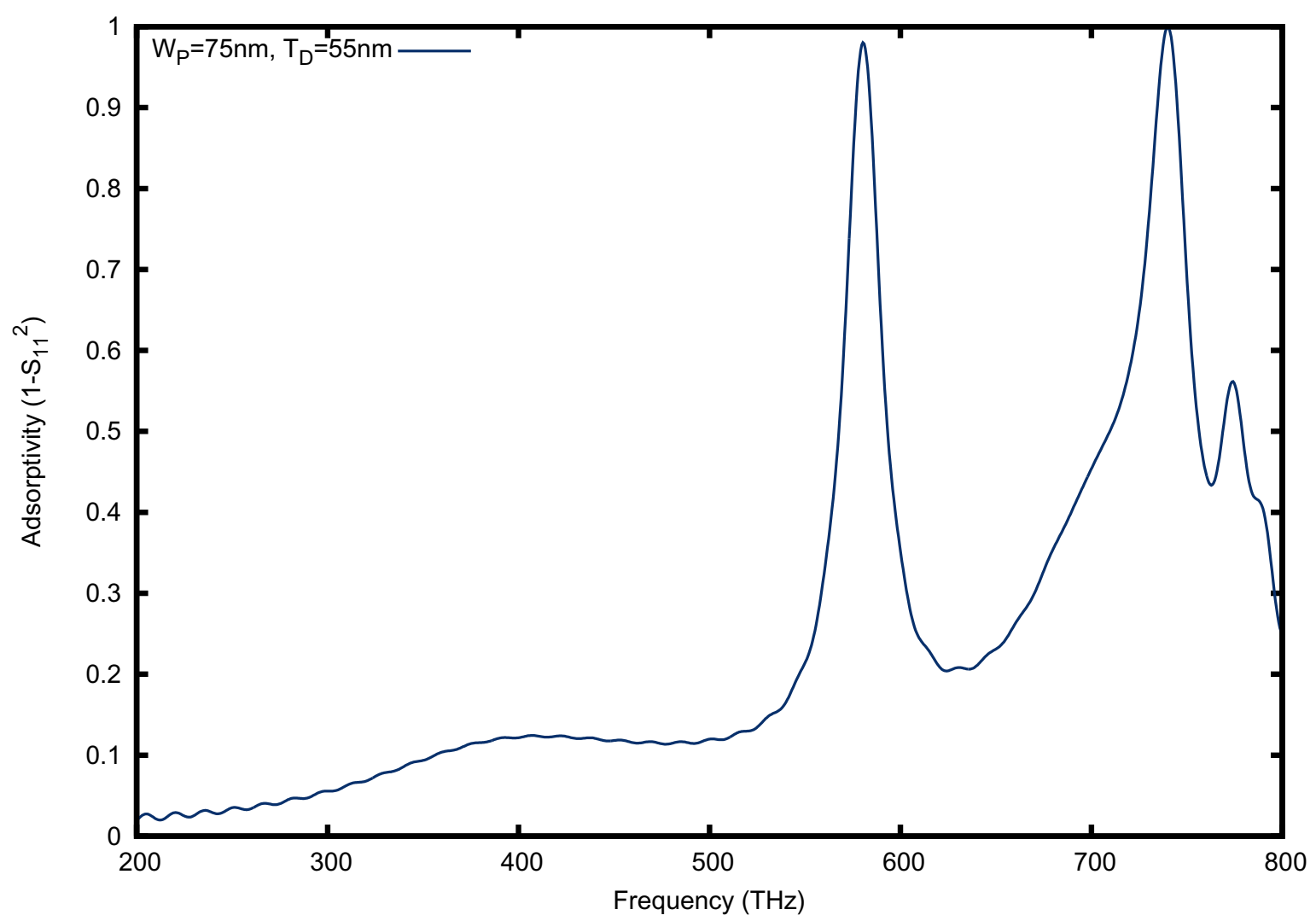

Figure 4.28 Results of Design D of a grating coupler where the peak resonance is apparent when the spacing between the particles is twice the particle diameter.

The fourth design is a grating coupling design that is illustrated by Design 4 in Figure 4.22. The grating couple is evident by removing the ground plane. It is also noted that the grating couple is evident when the spacing between the particles is twice the particle diameter. This is illustrated in Figure 4.28 where the spacing between particles is set to $75 \mathrm{~nm}$ and the particle spacing is $150 \mathrm{~nm}$. The result of the ground plane with the grating results in a perfect absorber. 


\subsection{Effects of Magnetic and Electric Resonances}

In order to further our understanding of the interaction effects, this study was focused on the effects of electric and magnetic resonances individually. The effects of electric resonance was studied by conducting a sweep in the direction of the electric field only, that is, $\hat{u}$, denoted as (L1) and likewise the effects of the magnetic resonance was studied by conducting a sweep in the direction of the magnetic field, that is, $\hat{v}$, denoted as (W1) as presented in Figures 4.29 a) and b) respectively with their respective parameters in Table 4.5 and 4.6. The dielectric thickness was chosen to be $30.33 \mathrm{~nm}$ which was the optimized thickness obtained from the investigation of Design A in Section 4.3 (see Figure 4.17). The absorptivity of the parameter L1 and W1 was studied from $50 \mathrm{~nm}(80 \mathrm{~nm})$ to $500 \mathrm{~nm}$ and the response calculated at each frequency in the visible regime. The results for the particle width of 50nm is presented in Figures 4.30 and Figures $4.31 \mathrm{a}$ ) and b).

The optimized results for both the L1 and W1 cases resulted when the dimensions of the design was $162.5 \mathrm{~nm} \times 500 \mathrm{~nm}$ for the substrate and dielectric length and width with a dielectric thickness of $30.33 \mathrm{~nm}$ producing four distinct peaks. From Figures $4.31 \mathrm{a}$ ) and b), it can be elucidated that the electric resonance is more dominant on the lower end of the spectrum whereas the effect of the magnetic resonance is more dominant on the center of the visible regime. Furthermore, it can be noticed that at 500nm, the electric resonance is absorbing sparsely regardless of the length of L1 and likewise around 650nm the magnetic resonance will be absorbing significantly regardless of the length of W1. Of importance to note, is the decoupled effect of the magnetic and the electric resonance, that is, in the regions where the absorptivity is governed by the electric resonance, the magnetic resonance exhibits no absorption and vice versa. 


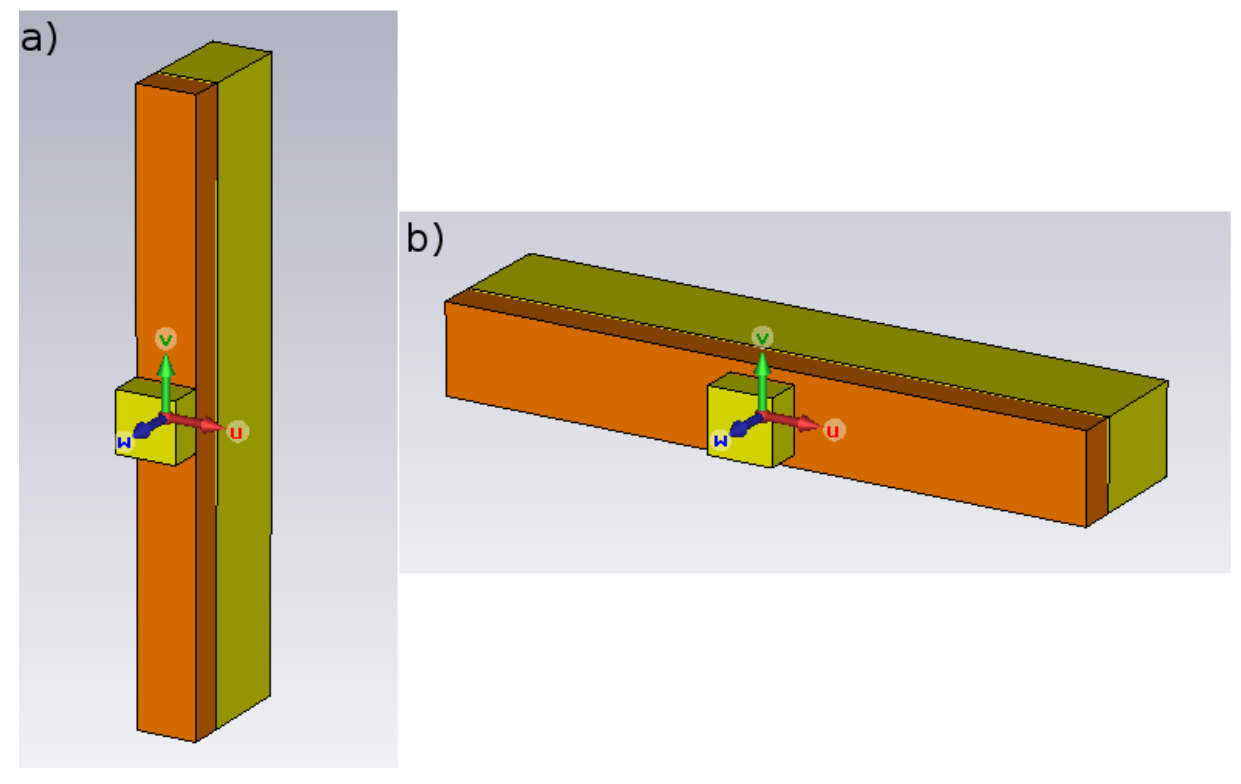

Figure 4.29 Sweep in the electric field direction (L1) to study the effect of electric resonance a) and sweep in the magnetic field direction (W1) to study the effect of the magnetic resonance b).

\begin{tabular}{|c|c|c|c|}
\hline \hline Constituent & Parameter & Description & Parameter Value $(\mathrm{nm})$ \\
\hline \hline \multirow{3}{*}{ Nanoparticle } & $W_{p}$ & particle width & $50(80)$ \\
& $L_{p}$ & particle length & $50(80)$ \\
& $T_{p}$ & particle thickness & 30 \\
\hline \multirow{3}{*}{ Dielectric } & $W_{d}$ & dielectric width & 500 \\
& $L_{d}$ & dielectric length & $50(80)$ \\
& $T_{d}$ & dielectric thickness & 30.33 \\
\hline \multirow{3}{*}{ Substrate } & $W_{s}$ & substrate width & 500 \\
& $L_{s}$ & substrate length & $50(80)$ \\
& $T_{s}$ & substrate thickness & 150 \\
\hline
\end{tabular}

Table 4.5 Critical parameters used for the (L1) sweep to study the effect of the electric resonance for a particle width of $50 \mathrm{~nm}(80 \mathrm{~nm})$. 


\begin{tabular}{|c|c|c|c|}
\hline \hline Constituent & Parameter & Description & Parameter Value $(\mathrm{nm})$ \\
\hline \hline \multirow{3}{*}{ Nanoparticle } & $W_{p}$ & particle width & $50(80)$ \\
& $L_{p}$ & particle length & $50(80)$ \\
& $T_{p}$ & particle thickness & 30 \\
\hline \multirow{3}{*}{ Dielectric } & $W_{d}$ & dielectric width & $50(80)$ \\
& $L_{d}$ & dielectric length & 500 \\
& $T_{d}$ & dielectric thickness & 30.33 \\
\hline \multirow{3}{*}{ Substrate } & $W_{s}$ & substrate width & $50(80)$ \\
& $L_{s}$ & substrate length & 500 \\
& $T_{s}$ & substrate thickness & 150 \\
\hline
\end{tabular}

Table 4.6 Critical parameters used for the (W1) sweep to study the effect of the magnetic resonance for a particle width of $50 \mathrm{~nm}(80 \mathrm{~nm})$.

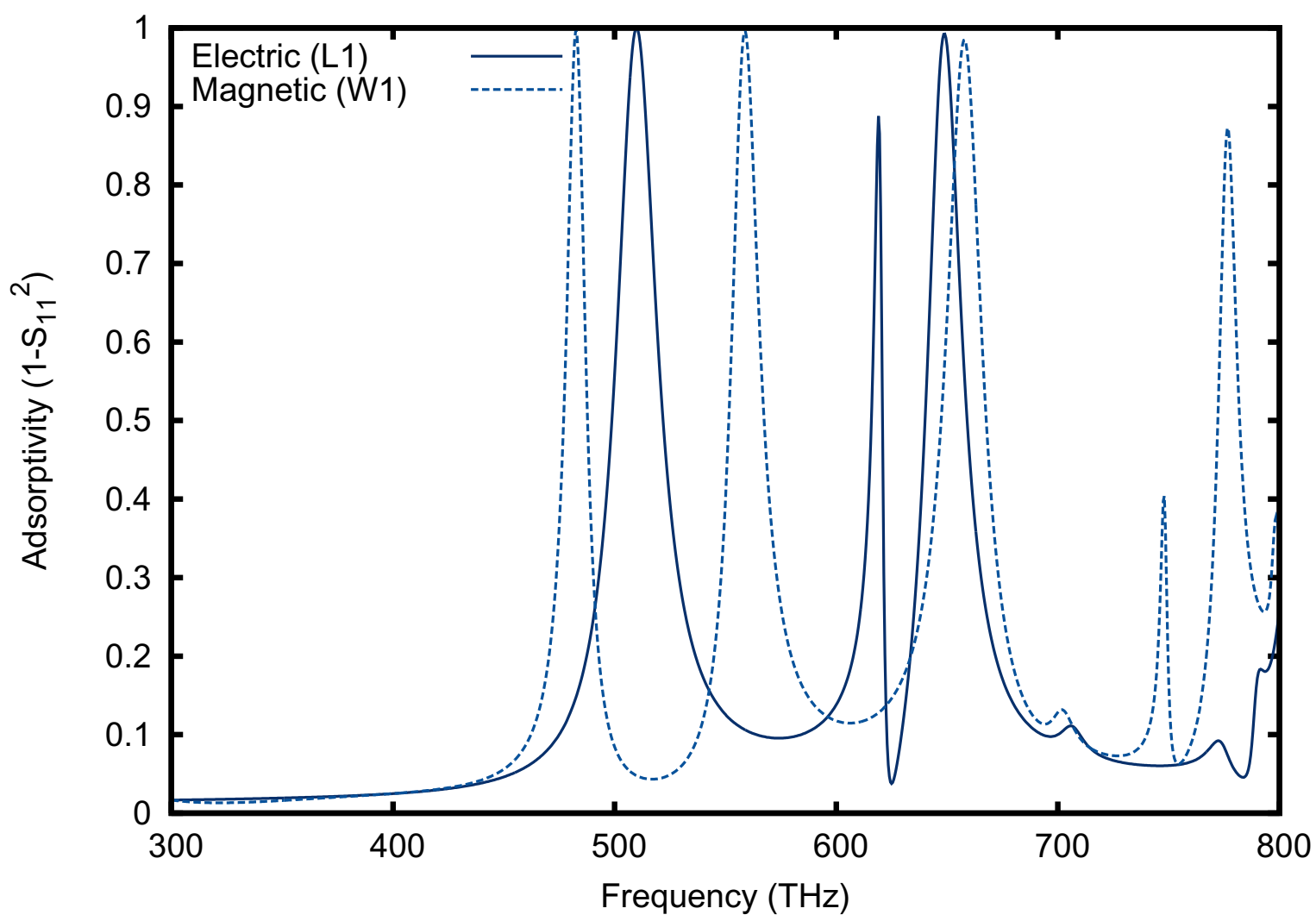

Figure 4.30 Combined absorption response of the electric and magnetic resonances. Figure 4.29 a) presents the absorption response by inducing a changing electric field and b) presents the absorption response by inducing a changing magnetic field. 

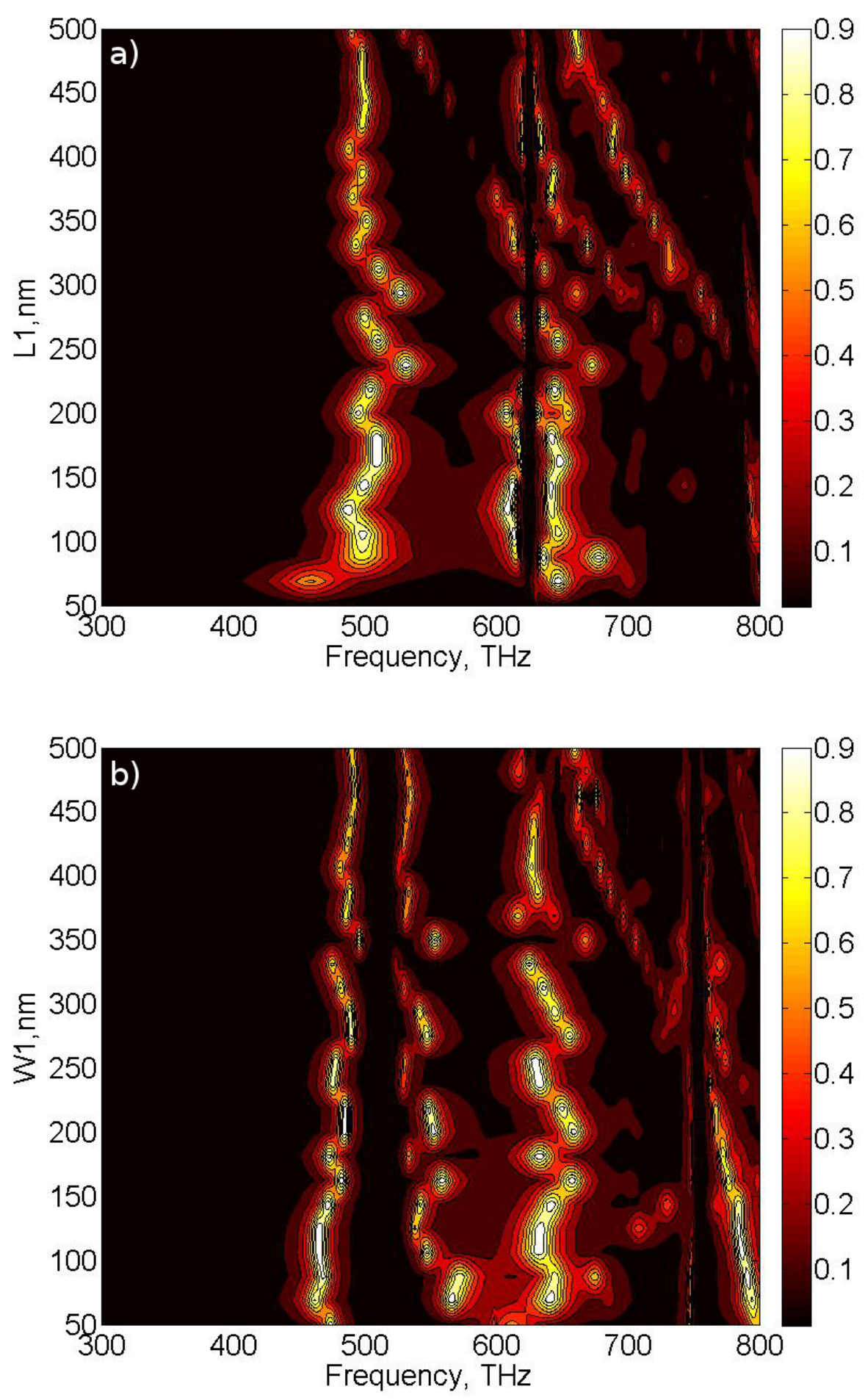

Figure 4.31 Contour representation of the combined absorption response of the electric and magnetic resonances for a particle width $W_{p}=50 \mathrm{~nm}$. 
Figure 4.32 shows the same simulation (L1 and W1 sweep) but for a particle width of $80 \mathrm{~nm}$ to study the interaction effects. When compared to the $50 \mathrm{~nm}$ particle width sweep, the $80 \mathrm{~nm}$ exhibits similar characteristics. The remarkable difference when comparing the L1 and W1 sweep for 50nm and 80nm, as presented in Figure 4.32, is that the absorption spectrum has red shifted. This behavior is consistent with our discussion of Design B (cavity effect) in Section 4.4 whereby the increase in particle width induced a decrease in the cavity size, hence, red shifting the absorption spectrum. The other major occurrence is that for L1 sweep of $80 \mathrm{~nm}$, not only the absorption spectrum has red shifted but it has also increased in strength as shown in Figure $4.32 \mathrm{c}$ ) with the strength increasing as the particle was sweeped further outwards. This is because when the range of L1 was increased, the behavior of Design A started resurfacing, that is, the absorption characteristics of the unit cell absorber was recaptured. This fact was further reinforced by the re-emergence of the bifurcation peaks visible clearly in Figures 4.32 a) and c) as the higher end of the visible regime when the separation distance between the particles has been increased significantly.

Figure 4.33 provides information on the electric and magnetic field distribution for Figure 4.29 b) for its four resonant modes as depicted in Figure 4.30. Figures 4.33 a), c), e) and g) indicate the almost negligible contribution of the electric field to the aborption response. Thus shifting our attention more towards the more dominant contribution by the magnetic resonance which is again consistent with our previous observations. In Figure 4.33 b), it can be observed that the fundamental mode is still induced by anti-parallel current resulting in the major contributor of heat generation and absorption in the dielectric component (see Section 4.2). For the second resonant mode, Figure $4.33 \mathrm{~d}$ ), the strength of the magnetic field has decreased but is still in the same phase as the first mode. Figure 4.33 f) has almost the same contribution to the absorption response with the exception that its phase has reversed. Finally, Figure $4.33 \mathrm{~h}$ ) illustrates the magnetic field resulting from neighboring cells. 

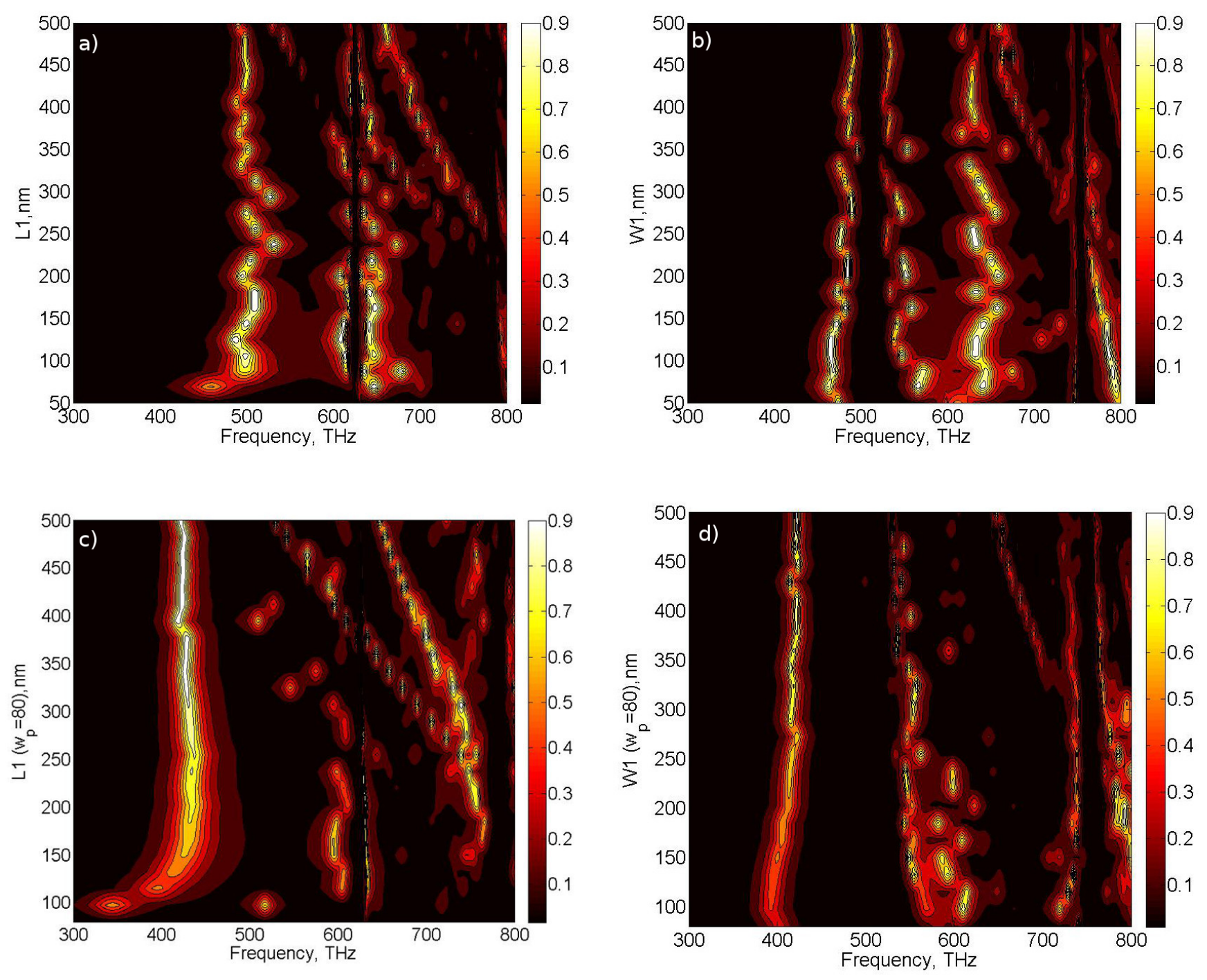

Figure 4.32 Contour representation of the combined absorption response of the electric and magnetic resonances for a particle width of $W_{p}=50 \mathrm{~nm}$ and $W_{p}=80 \mathrm{~nm}$. 

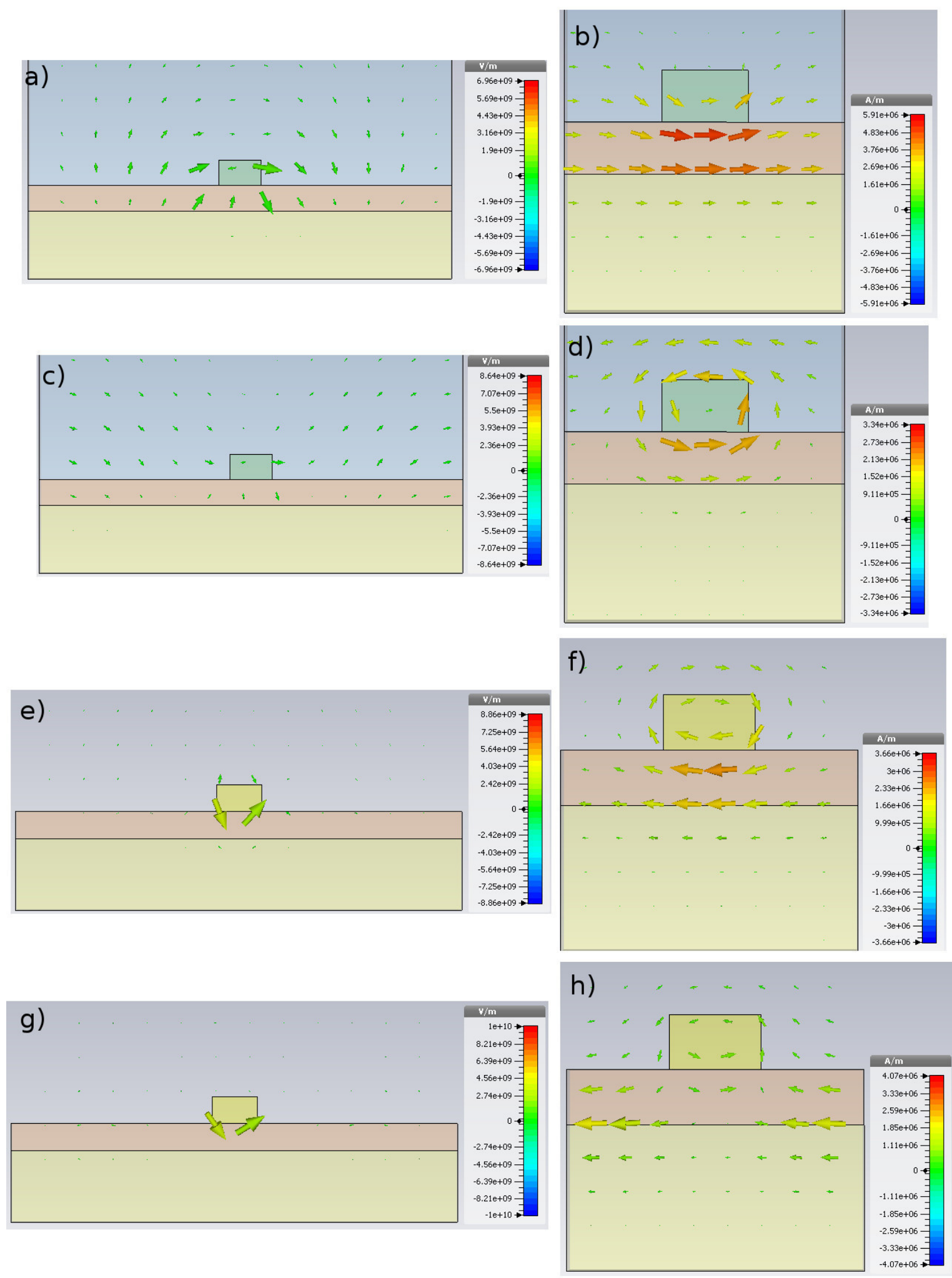

Figure 4.33 Electric and magnetic field distribution for the W1 sweep for resonant frequencies at $\omega_{1^{s t}}=482.5 \mathrm{THz}, \omega_{2^{n d}}=559 \mathrm{THz}, \omega_{3^{r d}}=658.2 \mathrm{THz}$, and $\omega_{4^{t h}}=776.53 \mathrm{THz}$. 
From the preceding sections, it can be elucidated that the optimized results has been obtained when the dielectric thickness of $30.33 \mathrm{~nm}$ was employed. Furthermore, it was observed that a rectangular construction of $162.5 \mathrm{~nm} \times 500 \mathrm{~nm}$ provides a decoupled electric and magnetic resonance with an absorptivity strength of almost $100 \%$. By exploiting this decoupling phenomena, a broadband absorber can be produced with periodic repeating rectangular elements as illustrated in Figure 4.34. This is our proposed design for the optimized thermal absorber.

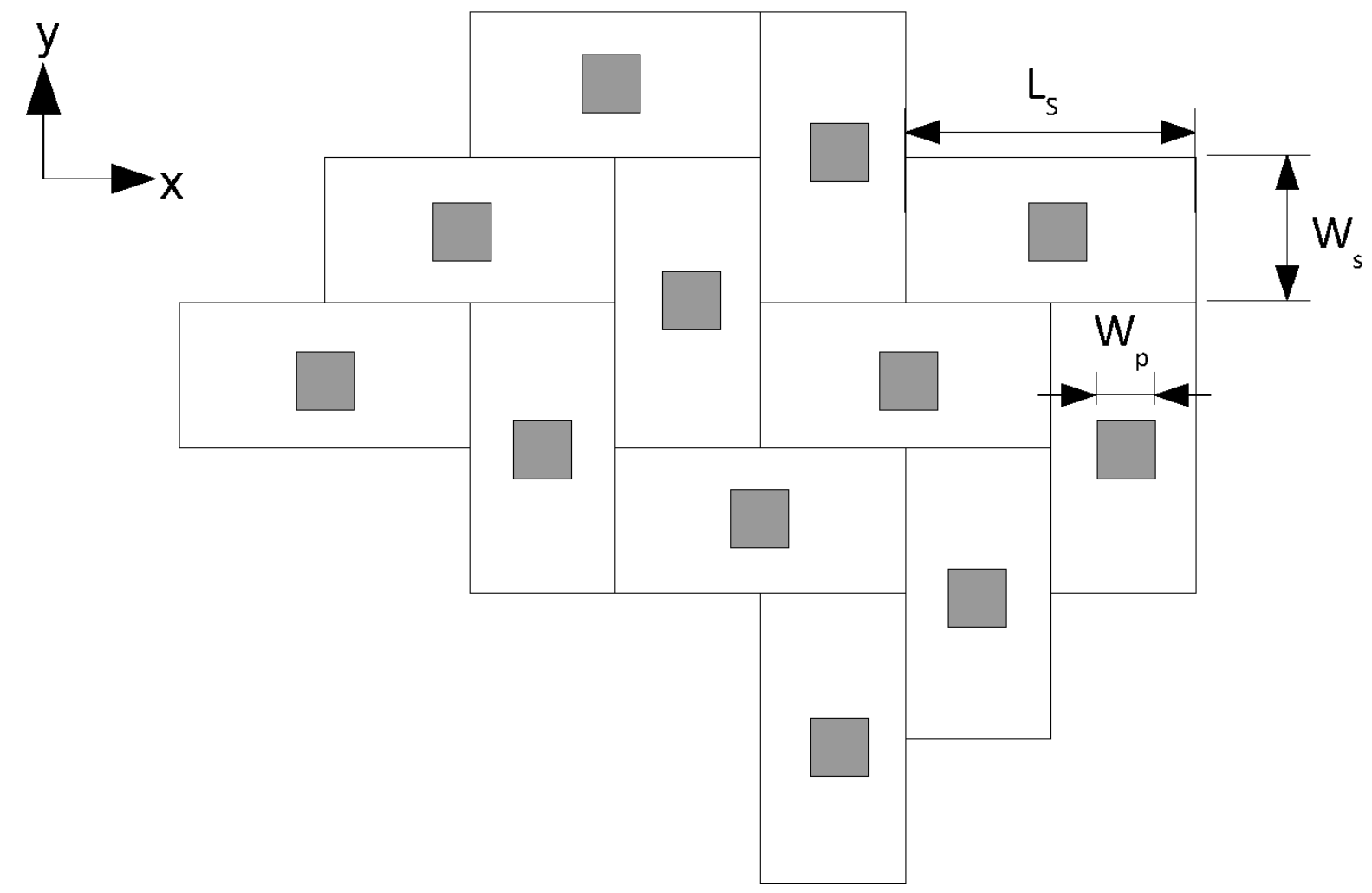

Figure 4.34 Proposed optimized thermal absorber for the unit-celled silver nano-particle of Design A. 


\subsection{Resonance information for the second order absorber}

This investigation was carried out to determine an optimal translation vector for a fractal arrangement plasmonic metamaterial. In order to be consistent with our previous findings, the dielectric thickness for this investigation was chosen as $30 \mathrm{~nm}$ with the particle dimensions taken as $W_{p} \times L_{p} \times T_{p}=50 \mathrm{~nm} \times 50 \mathrm{~nm} \times 30 \mathrm{~nm}$. This structure was labeled as the second order absorber, as illustrated in Figure 4.35, because of its continuity from the design of our perfect absorber (Desgin A). The simulations were carried out in the same manner as detailed in the previous sections whereby a parameter sweep was conducted for identical silver nano-particles moving diagonally away from the central nano-particle as illustrated in Figure $4.35 \mathrm{~b})$.
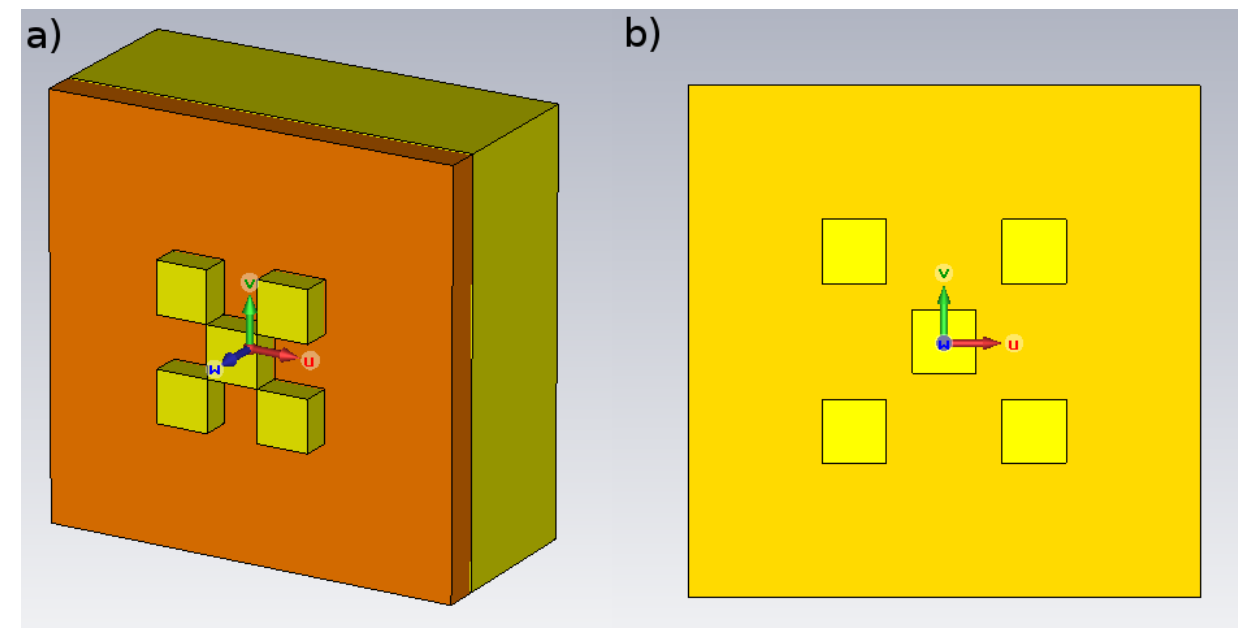

Figure 4.35 Perspective and front view of the optimized second order absorber.

The results are presented in Figure 4.36 which shows the presence of multi-resonances at the sub-wavelength scales in the visible regime. The appearance of the multi-resonances is a sure indicator of an increase in absorption intensity when compared to the singular frequency perfect absorber. Results further indicate the same interaction effects as discussed earlier as the identical silver nano-particles are swept further away. 


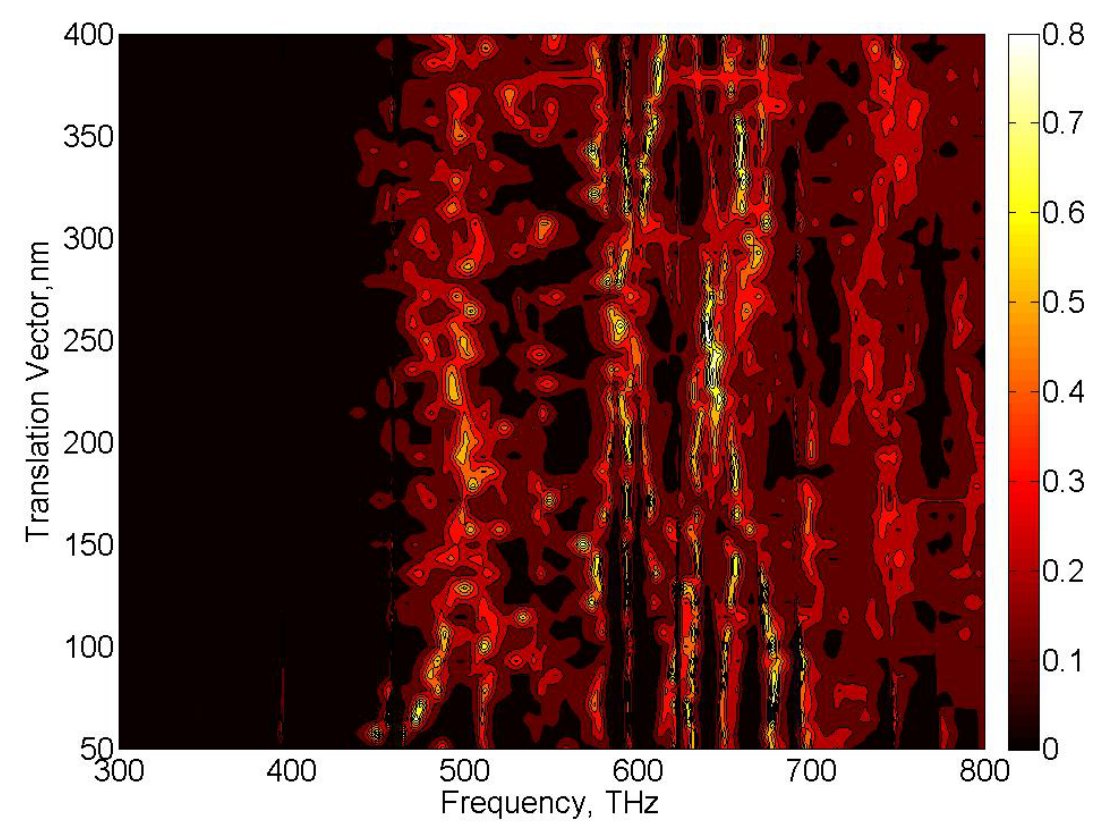

Figure 4.36 Contour representation of the absorption response for the second order absorber.

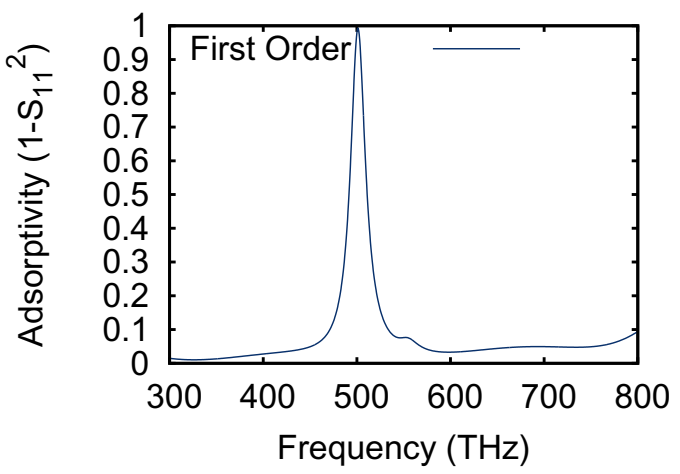

Figure 4.37 Singular resonant peak for the first order absorber.

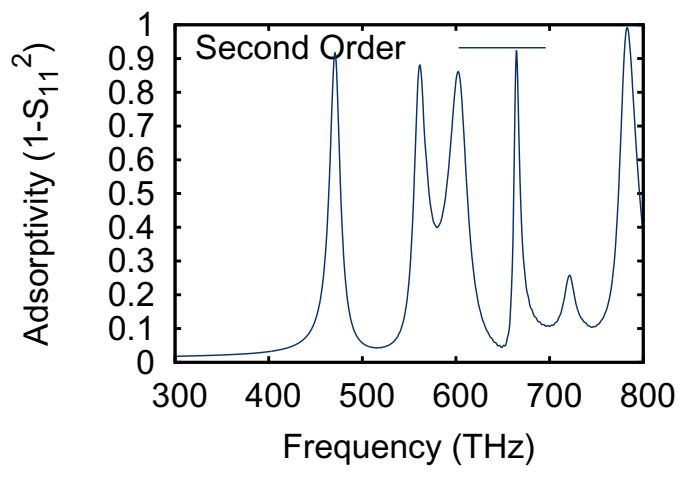

Figure 4.38 Multi-resonant peaks for the second order absorber

From the Figures 4.37 and 4.38, one can intuitively say that the absorption response has increased in magnitude from the first order absorber to the second order absorber. In order to compare this increase quantitatively, Equation 4.1 and 4.2 are utilized, where $\mathrm{A}(\omega)$ $=1$, and the area under the absorption vs. frequency curve is evaluated at half width and 
half max.

$$
\begin{gathered}
I_{\text {single }}=A_{\text {single }} \times 1 \times \frac{1}{2}_{\text {width }} \frac{1}{\text { max }} \\
I_{\text {multiple }}=\left(A_{1} \times 1 \times \frac{1}{2}{ }_{\text {width }} \frac{1}{\text { max }}\right)_{\text {peak } 1}+\left(A_{2} \times 1 \times \frac{1}{2}{ }_{\text {width }} \overline{2}_{\text {max }}\right)_{\text {peak } 2}+\ldots
\end{gathered}
$$

The absorption intensity for the singular peak (first order absorber) was calculated to be 5.434 whereas the absorption intensity for the multi-peaks (second order absorber) was calculated to be 38.736. From here, it was elucidated that the absorptivity of the second order absorber was increase by seven fold. Thus, it is confirmed that the absorption response increases with increasing order of absorbers, that is, an increase in the number of resonant peaks is proportional to the increase in the absorptivity of the absorber. 


\subsection{Resonance information for the third order absorber}

In order to further our theoretical understanding of increasing order absorbers, a second and third order absorber was constructed as illustrated in Figure $4.39 \mathrm{c})$. The simulation procedure was undertaken in an identical manner as discussed in the previous sections. However, here the translation vector was not identical. It was $2 \times W_{p}$ in the direction of the $\vec{E}$ field and $W_{p}$ in the direction of the $\vec{H}$ field. The results are presented in Figure 4.40. The results indicate an increase in the bandwidth centered around the fundamental frequency at $\omega_{0}=500 \mathrm{THz}$. Also there is an increase in the number of resonant peaks around the fundamental peak. These observations are aligned with our previous discussion that an increase in the absorber order will cause an increase in the bandwidth as well as an improvement in the excitation strength. This is an example of a non-symmetrical construction whereby the nonsymmetrical nature helps to selectively influence the incident electromagnetic radiation by creating absorptivity response as a function of electric and magnetic resonances differently. That is, it allows for individual tuning of the magnetic and electric responses.
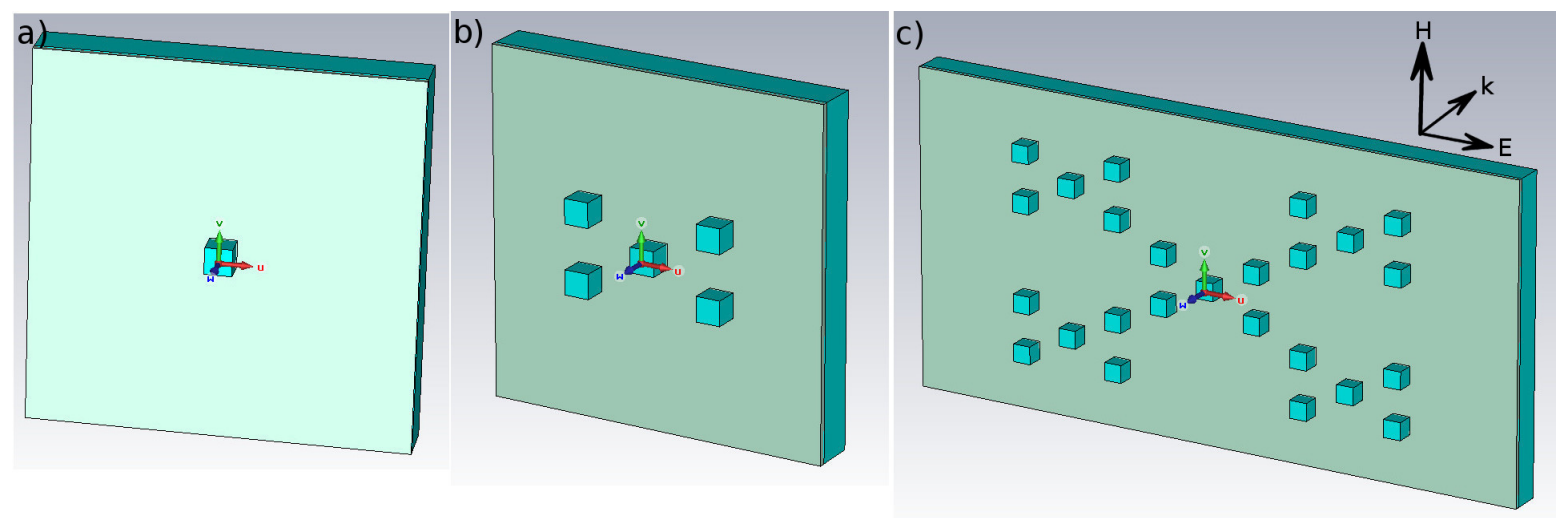

Figure 4.39 Perspective view of a third order absorber as an evolution from the first order absorber. 


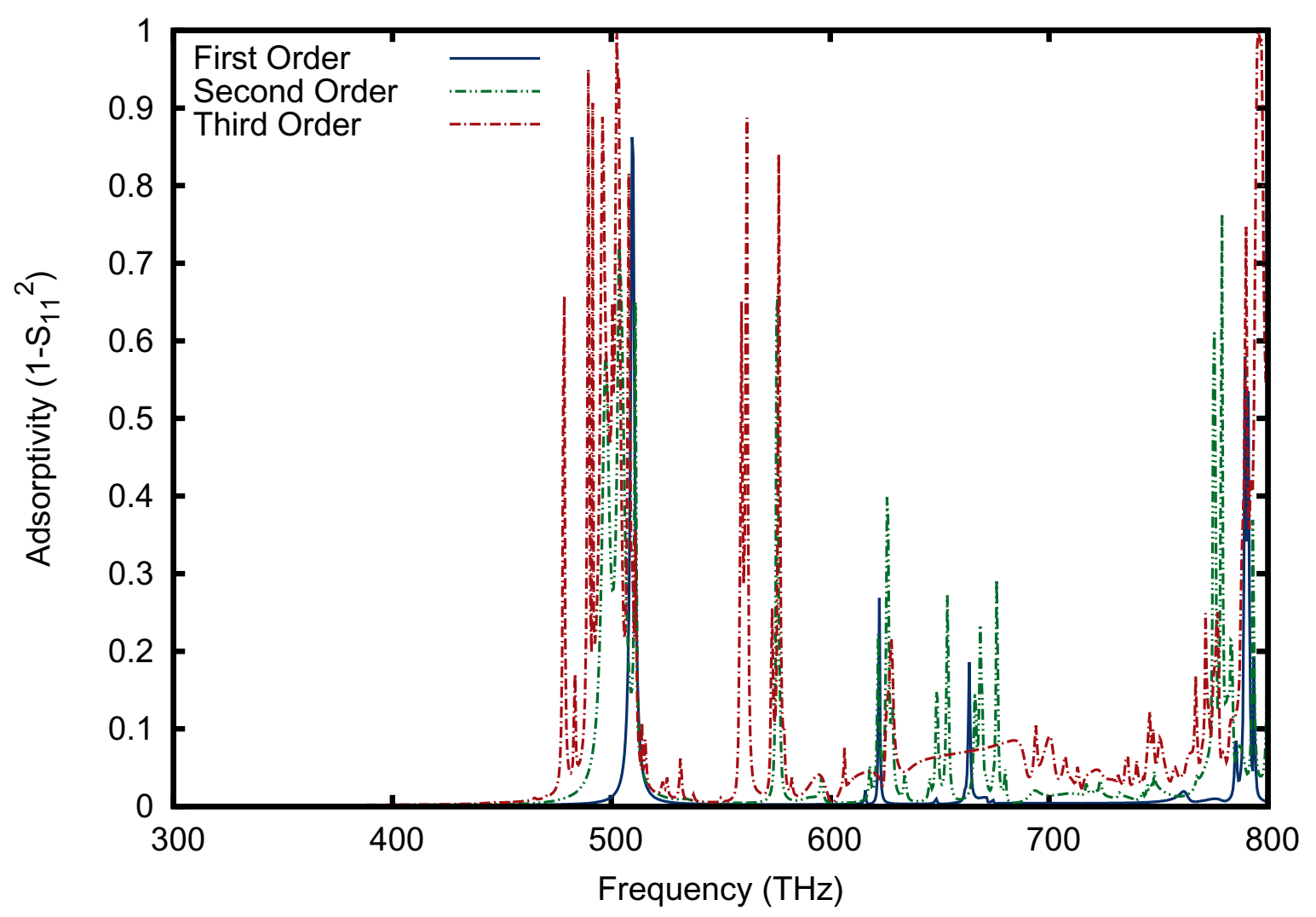

Figure 4.40 A comparison between the absorptivity response of the first order, second order and third order absorber designs. 


\section{CHAPTER 5}

\section{CONCLUSION}

\subsection{Summary of Research}

The purpose of this research was to develop a perfect but realizable broadband thermal absorber from plasmonic metamaterials with the capacity to absorb and confine the maximum about of solar energy reaching the earth's surface.

The first quarter of the research was dedicated to understanding the behavior of the perfect absorber. From the results it was elucidated that the fundamental mode of the perfect absorber was governed by anti-parallel current and the absorbance was dominated by the magnetic resonance. These results were verified by studying the field, current and power distribution throughout the structure. The magnetic resonance was shown to be responsible for the greatest contribution of heat generation and confinement in the dielectric or insulator

elements of this structure. Furthermore, the results indicated that the heat generated could be concentrated to specific locations in the perfect absorber. In this case, the heat was concentrated directly underneath the silver nano-particle at the metal-dielectric interface.

The second quarter of the research was focused towards the understanding of the plasmonic response to topological changes of the silver nano-particle in the dielectric environment. The results illustrated a bifurcation of the absorptivity as a function of particle width not necessary at an integer fraction of the particle width. Results also found a moderate but less significant shift in the peak absorptivity with change in dielectric thickness. These results have the aim in providing guidance in the experimental fabrication of highly geometric nanoparticle plasmonic devices. These results suggest that a large diameter particle $(>90 \mathrm{~nm})$ can be deposited on top of a controlled thickness dielectric material for tuned optical frequency response. 
The third quarter investigated the influences of neighboring particles on the nearly perfect absorption of electromagnetic radiation between 200 to $800 \mathrm{THz}$. In order to optimize the interaction between the neighboring particles their fundamental interactions were broken down into three designs and studies independently. Those three designs were 1) anti-parallel currents, 2) cavity resonances, 3) effective medium, and 4)grating coupling. Given the identification of these four interactions the influence on their geometry identified how the peak resonances shift. With this knowledge of neighboring interaction the plasmonic response of complex designs that involve neighboring interactions can be used to engineer a desired response.

The last quarter of the research dealt with the individual effects of magnetic and electric resonances on the absorption response of the thermal absorber. Results indicated a decoupling effect with the magnetic resonances absorbing in the central region of the visible regime and the electric resonances absorbing at the outer limits of the visible regime bordering in the infrared regime. These decoupling effects were exploited to propose a broadband thermal absorber. The increase in the order of the resonance peaks were observed and a seven fold increase in the absorption intensity was determined when compared to the perfect absorber. Further investigations reinforced the emergence of the aforementioned birfucation peaks and the shifting of the absorption peaks with changes in the nano-particle width.

Furthermore, a second order absorber was investigated for an optimal particle distance in a fractal arrangement. The optimal translation vector for the most efficient absorption response was determined. Results indicated the emergence of multi-resonance with absorption responses at the sub-wavelength scales. The presence of multi-resonances is indicative of the increasing absorption intensity when compared to the perfect absorber in a broadband response. 


\subsection{Future Directions}

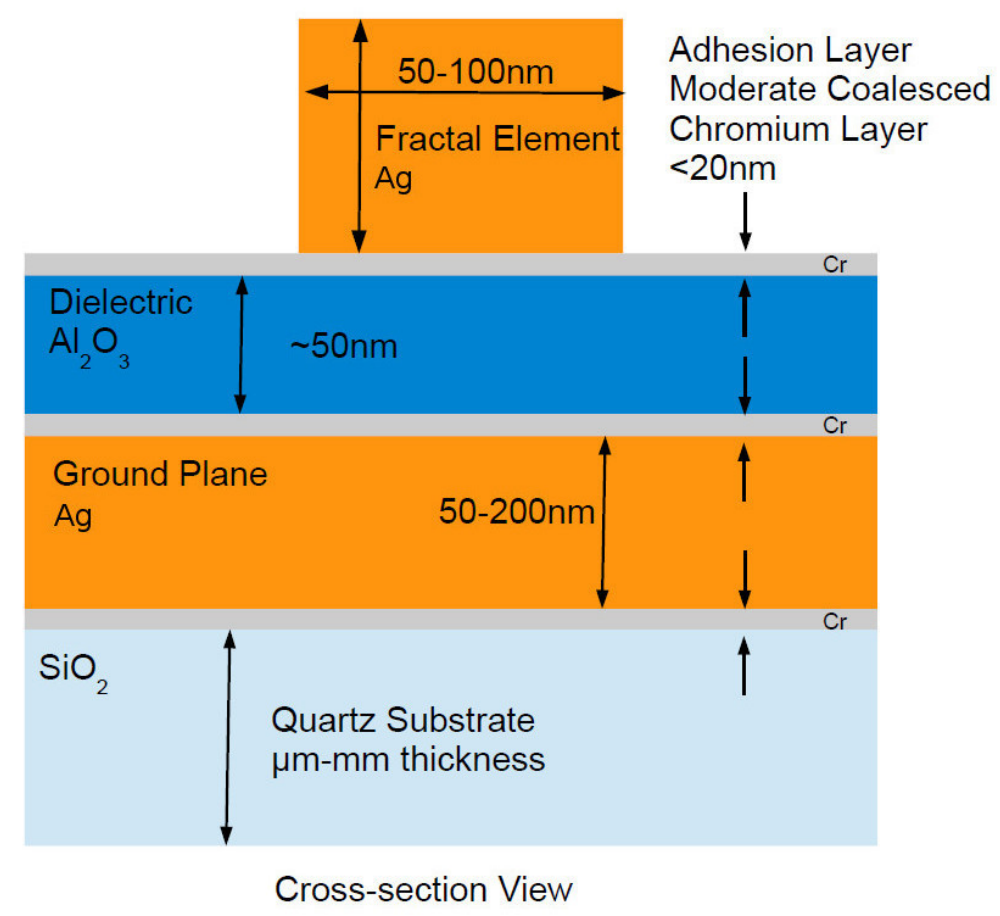

Figure 5.1 Proposed plasmonic metamaterial thermal absorber construction for Design A.

The future goals for this research are to fabricate and realize the absorber for Design A as shown in Figure 5.1. The techniques that would be required for this undertaking are: 1) Physical vapor deposition of silver and chromium, 2) E-beam lithiography, 3) Plasma atomic layer deposition of aluminium oxide and e) Etching to implant the silver nano particle on the center of the domain of the thermal absorber. These processes would be carried out in a bottom up approach. Once the construction of the thermal absorber has been accomplished, a comparison between the simulated and experimental results can be conducted.

Furthermore, we are still going to target approaches to broaden the response further. This would be carried out by investigating an even higher order absorber, that is, 
a fourth order absorber. So far simulations were only performed on a three level design, metal-dielectric-metal construction. However, we would also like to perform a multi-layer design, that is, metal-dielectric-metal-dielectric-metal, construction with the hypothesis that an increase in layers will lead to a reduction in reflection as a result of destructive interference as light rays will be reflected from each respective interfaces. Moreover, we also plan to investigate non-symmetrical absorbers. Till to date quarter symmertical absorbers have been theoretically studied in this research in second order absorber and third order absorber where the translation vector was identical in the $\mathrm{x}$ and $\mathrm{y}$ direction. The non-symmetry will be achieved by employing a translation vector for different values in the $\mathrm{x}$ and $\mathrm{y}$ direction. Finally, we aspire to further this research by the implementation of a completely different design for the perfect absorber, that is, a three petal-shaped flower structure with increasing orders with an identical thought process that this design will lead to broadening of the absorptivity response by representing all the sub-wavelength scales in the visible regime. 


\section{BIBLIOGRAPHY}

[1] P. Lorrain and D. R. Corson. Electromagnetism Principles and Applications, chapter 20. W. H. Freeman and Company, 1969.

[2] Z. M. Zhang. Nano/Microscale Heat Transfer, chapter 8. The McGraw-Hill Companies, Inc., 2007.

[3] A. Hunter Fanney. Mobile solar tracker facility, November 2011.

[4] K. N. Liou. An Introduction to Atmospheric Radiation Second Edition, chapter 2. Elsevier, 2002.

[5] Plasmonics From Basics to Advanced Topics. New York:Springer, 2012.

[6] T. J. Cui, D. R. Smith, and R. Liu. METAMATERIALS Theory, Design and Applications, chapter 1. Springer, 2010.

[7] G. Lipworth. Magnetic metamaterial superlens for increased range wireless power transfer. Scientific Reports, 4:3642, 2014. PMID: 24407490.

[8] K. Shiraga, Y. Ogawa, K. Hattori, T. Suzuki, N. Kondo, A. Irisawa, and M. Imamura. Metamaterial application in sensing for living cells. In Solid-State Sensors, Actuators and Microsystems (TRANSDUCERS EUROSENSORS XXVII), 2013 Transducers Eurosensors XXVII: The 17th International Conference on, pages 1198-1201, June 2013.

[9] J. B. Pendry, D. Schurig, and D. R. Smith. Controlling electromagnetic fields. Science, 312(5781):pp. 1780-1782, 2006.

[10] Y. Tachibana, K. Kusunoki, T. Watanabe, K. Hashimoto, and H. Ohsaki. Optical properties of multilayers composed of silver and dielectric materials. Elsevier, 2003. 
[11] O. Benson. Assembly of hybrid photonic architectures from nanophotonic constituents. Nature, 480:193-199, 2011.

[12] K. A. Willets, Van D., and Richard P. Localized surface plasmon resonance spectroscopy and sensing. Annual Review of Physical Chemistry, 58(1):267-297, 2007. PMID: 17067281.

[13] N. I. Landy, S. Sajuyigbe, J. J. Mock, D. R. Smith, and W. J. Padilla. Perfect metamaterial absorber. Phys. Rev. Lett., 100:207402, May 2008.

[14] P.V. Tuong, J.W. Park, V.D. Lam, K.W. Kim, H. Cheong, W.H. Jang, and Y.P. Lee. Simplified perfect absorber structure. Computational Materials Science, 61(0):243 247, 2012.

[15] M. Li, S. Liu, L. Guo, H. Lin, H. Yang, and B. Xiao. Influence of the dielectric-spacer thickness on the dual-band metamaterial absorber. Optics Communications, 295(0):262 $-267,2013$.

[16] C. M. Watts, X. Liu, and W. J. Padilla. Metamaterial electromagnetic wave absorbers. Advanced Materials, 24(23):OP98-OP120, 2012.

[17] Y. Cheng, Y. Nie, and R. Gong. A polarization-insensitive and omnidirectional broadband terahertz metamaterial absorber based on coplanar multi-squares films. Optics and Laser Technology, 48(0):415 - 421, 2013.

[18] K. Mizuno, J. Ishii, H. Kishida, Y. Hayamizu, S. yasuda, D. N. Futaba, M. Yumura, and K. Hata. A black body absorber from vertically aligned single-walled carbon nanotubes. PNAS, 106(15):60446047, April 2009.

[19] M. Blanco-Muriel, Alarcn. P., Diego C., T. Lpez-Moratalla, and M. Lara-Coira. Computing the solar vector. Solar Energy, 70(5):431 - 441, 2001. 
[20] L.L. Vant-Hull and A.F. Hildebrandt. Solar thermal power system based on optical transmission. Solar Energy, 18(1):31 - 39, 1976.

[21] S. Redi, G. S. Aglietti, A. R. L. Tatnall, and T. Markvart. An evaluation of a high altitude solar radiation platform. 2010.

[22] I. Reda and A. Andreas. Solar position algorithm for solar radiation applications. Solar Energy, 81(6):838 - 849, 2007.

[23] P.I. Cooper. The absorption of radiation in solar stills. Solar Energy, 12(3):333 - 346, 1969.

[24] O. Hess, J.B. Pendry, S.A. Maier, R.F. Oulton, J.M. Hamm, and K.L. Tsakmakidis. Active nanoplasmonic metamaterials. Nature Materials, 2012.

[25] J. Wang, S. Qu, Z. Xu, J. Zhang, H. Ma, Y. Yang, and C. Gu. Broadband planar lefthanded metamaterials using split-ring resonator pairs. Photonics and Nanostructures Fundamentals and Applications, 7(2):108 - 113, 2009.

[26] H. Xu, G. Wang, C. Zhang, Q. Liu, Z. Xu, X. Chen, and D. Zhai. Multi-band left-handed metamaterial inspired by tree-shaped fractal geometry. Photonics and Nanostructures - Fundamentals and Applications, 11(1):15 - 28, 2013.

[27] P.V. Tuong, V.D. Lam, J.W. Park, E.H. Choi, S.A. Nikitov, and Y.P. Lee. Perfectabsorber metamaterial based on flower-shaped structure. Photonics and Nanostructures - Fundamentals and Applications, 11(1):89 - 94, 2013.

[28] X. Liu, T. Starr, A. F. Starr, and W. J. Padilla. Infrared spatial and frequency selective metamaterial with near-unity absorbance. Phys. Rev. Lett., 104:207403, May 2010.

[29] A. Boltasseva and H. A. Atwater. Low-loss plasmonic metamaterials. Science, 331(6015):290-291, 2011. 
[30] V. S. Slobodan, M. N. Branislav, and M. I. Milan. Conformal cubical 3d transformationbased metamaterial invisibility cloak. J. Opt. Soc. Am. A, 30(1):7-12, Jan 2013.

[31] L. Shengying, W. Qun, and Z. Kuang. Dispersion effect on electromagnetic properties of metamaterials invisibility cloak. In Cross Strait Quad-Regional Radio Science and Wireless Technology Conference (CSQRWC), 2011, volume 1, pages 226-229, July 2011.

[32] S. Samuel, editor. Microwave Antenna Theory and Design. Electromagnetic Waves. Institution of Engineering and Technology, 1984.

[33] S. Zhou. Electrodynamic Theory of Superconductors. Electromagnetic Waves. Institution of Engineering and Technology, 1991.

[34] V. P. Shestopalov and Y. V. Shestopalov. Spectral Theory and Excitation of Open Structures. Electromagnetic Waves. Institution of Engineering and Technology, 1996.

[35] B. R. Cooper, H. Ehrenreich, and H. R. Philipp. Optical properties of noble metals. ii. Phys. Rev., 138:A494-A507, Apr 1965.

[36] L. Novotny and B. Hech. Priciple of Nano-Optics, chapter 12. Cambridge University Press, 2006.

[37] D.E. Aspnes. Plasmonics and effective-medium theories. Thin Solid Films, 519(9):2571 - 2574, 2011. 5th International Conference on Spectroscopic Ellipsometry (ICSE-V).

[38] S. J. Youn, T. H. Rho, B. I. Min, and Kwang S. Kim. Extended drude model analysis of noble metals. physica status solidi (b), 244(4):1354-1362, 2007.

[39] J. Hao, L. Zhou, and M. Qiu. Nearly total absorption of light and heat generation by plasmonic metamaterials. Phys. Rev. B, 83:165107, Apr 2011.

[40] T. Koschny, M. Kafesaki, E. N. Economou, and C. M. Soukoulis. Effective medium theory of left-handed materials. Phys. Rev. Lett., 93:107402, Sep 2004. 
[41] W. Kreher. Piezoelectricity, effective medium theories. volume 114 of Springer Series in Materials Science, pages 517-533. Springer Berlin Heidelberg, 2008.

[42] G. V. Naik, Jingjing Liu, A. V. Kildishev, V. M. Shalaev, and A. Boltassevaa. Demonstration of alzno as a plasmonic component for near-infrared metamaterials. Proceedings of the National Academy of Sciences of the United States of America, 2012.

[43] V. P. Drachev, U. K. Chettiar, A. V. Kildishev, H. K. Yuan, W. Cai, and V. M. Shalaev. The silver dielectric function in plasmonic metamaterials. Opt. Express, 16(2):11861195, Jan 2008.

[44] R. Ruppin. Electromagnetic energy density in a dispersive and absorptive material. Physics Letters A, 299(23):309 - 312, 2002.

[45] P.L. Richards. Bolometers for infrared and millimeter waves. Applied Physics Reviews, 1994.

[46] N. T. Tung, T. X. Hoai, V. D. Lam, J. W. Park, V. T. Thuy, and Y. P. Lee. Perfect impedance-matched left-handed behavior in combined metamaterial. European Physical Journal B - Condensed Matter, 74(1):47 - 51, 2010.

[47] H. Wakatsuchi, S. Greedy, C. Christopoulos, and J. Paul. Customised broadband metamaterial absorbers for arbitrary polarisation. Opt. Express, 18(21):22187-22198, Oct 2010.

[48] P. Maraghechi and A. Y. Elezzabi. Enhanced tetrahertz radiation emission from plasmonic complementary sierpinski fractal emitters. Opt. Express, 18(26):27336-27345, Dec 2010.

[49] G. Volpe, G. Volpe, and R. Quidant. Fractal plasmonics: subdiffraction focusing and broadband spectral response by a sierpinski nanocarpet. Opt. Express, 19(4):3612-3618, Feb 2011. 
[50] W. Zhu, X. Zhao, B. Gong, L. Liu, and B. Su. Optical metamaterial absorber based on leaf-shaped cells. Applied Physics A, 102(1):147-151, 2011.

[51] K. B. Alici, A. B. Turhan, C. M. Soukoulis, and E. Ozbay. Optically thin composite resonant absorber at the near-infrared band: a polarization independent and spectrally broadband configuration. Opt. Express, 19(15):14260-14267, Jul 2011.

[52] K. Aydin, V. E. Ferry, R. M. Briggs, and H. A. Atwater. Broadband polarizationindependent resonant light absorption using ultrathin plasmonic super absorbers. Nature Communications, November 2011.

[53] N. Berkovitch and M. Orenstein. Broadband plasmonic metamaterials. In Lasers and Electro-Optics (CLEO), 2012 Conference on, pages 1-2, May 2012.

[54] S. Seely and A. D. Poularikas. Electromagnetics Classical and Modern Theory and Applications, chapter 8. Marcel Dekker, Inc, 1979.

[55] J. C. Slater and N. H. Frank. Electromagnetism, chapter 8. Dover Publication, Inc, 1969. 